\title{
Transsynaptic Delineation of the Rabbit Eyeblink Premotor Pathway: Identifying Anterior Interpositus Neurons and Their Synaptic Changes as a Function of Learning
}

Jimena Gonzalez-Joekes

Follow this and additional works at: https://researchrepository.wvu.edu/etd

\section{Recommended Citation}

Gonzalez-Joekes, Jimena, "Transsynaptic Delineation of the Rabbit Eyeblink Premotor Pathway: Identifying Anterior Interpositus Neurons and Their Synaptic Changes as a Function of Learning" (2014). Graduate Theses, Dissertations, and Problem Reports. 5693.

https://researchrepository.wvu.edu/etd/5693

This Dissertation is protected by copyright and/or related rights. It has been brought to you by the The Research Repository @ WVU with permission from the rights-holder(s). You are free to use this Dissertation in any way that is permitted by the copyright and related rights legislation that applies to your use. For other uses you must obtain permission from the rights-holder(s) directly, unless additional rights are indicated by a Creative Commons license in the record and/ or on the work itself. This Dissertation has been accepted for inclusion in WVU Graduate Theses, Dissertations, and Problem Reports collection by an authorized administrator of The Research Repository @ WVU.

For more information, please contact researchrepository@mail.wvu.edu. 


\title{
Transsynaptic Delineation of the Rabbit Eyeblink Premotor Pathway: Identifying Anterior Interpositus Neurons and Their Synaptic Changes as a Function of Learning
}

\section{Jimena Gonzalez-Joekes}

Dissertation submitted to the School of Medicine and the Department of Neurobiology and Anatomy at West Virginia University in partial fulfillment of the requirements for the degree of

\section{Doctor of Philosophy in Neuroscience}

\author{
Approved by Doctoral Committee: \\ Bernard G. Schreurs, PhD, Chair \\ Albert S. Berrebi, PhD \\ Adrienne K. Salm, PhD \\ Maxim Sokolov, $\mathrm{PhD}$ \\ George A. Spirou, PhD \\ Department of Neurobiology and Anatomy \\ Morgantown, West Virginia \\ 2014
}

Copyright 2014

Jimena Gonzalez-Joekes 


\section{Abstract}

\section{Transsynaptic Delineation of the Rabbit Eyeblink Premotor Pathway: Identifying Anterior Interpositus Neurons and Their Synaptic Changes as a Function of Learning}

\section{Jimena Gonzalez-Joekes}

The main goal of this dissertation was to identify eyeblink projection neurons of the anterior interpositus nucleus (AIN) and to examine learning-specific changes in excitatory and inhibitory somatic synapses after eyeblink conditioning (EBC). In order to identify the specific subset of AIN neurons involved in EBC, a retrograde transneuronal viral tracer was injected into the eyelid (orbicularis oculi muscle) of the rabbit to reveal hierarchical chains of synaptically connected neurons involved in the eyeblink response. Experiment 1 was designed to provide a complete delineation of the rabbit eyeblink premotor pathway and to characterize the multiple neuronal types in the AIN that are involved in EBC and their possible interactions. Relevant structures involved in the generation or modulation of the rabbit eyeblink response were identified and possible sites of plasticity were revealed. First-order eyeblink motoneurons were found ipsilaterally in the dorsolateral facial nucleus. Second-order premotor neurons were found in the contralateral red nucleus and in different reticular, trigeminal, auditory, and vestibular nuclei. Third-order eyeblink premotor neurons were found in the pons, midbrain, and in the cerebellum, including dorsolateral AIN and rostral fastigial nucleus. Fourth-order neurons were found in Purkinje cells of the cerebellar cortex in lobule HVI and in lobule I. Eyeblink premotor neurons of the AIN were further characterized based on their neurotransmitter immunoreactivity which showed that glutamatergic eyeblink projection neurons are modulated by three different types of inhibitory interneurons that form a functional eyeblink microcomplex. Experiment 2 was designed to examine the effect of EBC on the number of excitatory and inhibitory somatic synapses in eyeblink projection neurons of the AIN. A coordinated increase in the number of 
excitatory and inhibitory Purkinje cell somatic synapses was observed in subjects in the experimental group that received paired delay EBC. These results support a parallel and correlated mechanism of cerebellar learning mediated by excitatory inputs presumably from mossy fibers and inhibitory inputs from Purkinje cells. In contrast, control subjects that received unpaired stimulus presentations showed an increase in the number of inhibitory somatic synapses originating from local interneurons, suggesting a possible role of feedback inhibition that may also explain the detrimental effect of unpaired exposure on subsequent learning. Another important finding was an increase in the somatic surface area of eyeblink projection neurons as a function of learning that was highly correlated with the number of somatic synapses; suggesting that synaptic remodeling is a bidirectional process that entails proportional structural alterations on the postsynaptic neuron. To our knowledge, this is the first time that the identity and function of specific nuclear neurons and their associated synaptic changes during learning can be linked to a behavior. 


\section{Acknowledgements}

This study was possible thanks to the support from many people. First of all, I would like to thank my advisor and committee Chair, Professor Dr. Bernard G. Schreurs for his advice, support, and guidance through all facets of this Dissertation. This work would have not been possible without his help. I would like to thank the members of my committee: Dr. Adrienne Salm, Dr. Albert Berrebi, Dr. George Spirou, and Dr. Maximum Sokolov for their advice and guidance in the development of the experimental design and its execution. Also, I would like to thank Dr. Fred Minnear and the Office of Research and Graduate Education for their support.

I would like to thank all the members in our lab, past and present, for their continuous support, especially Dr. Lauren Burhans for her advice, Carrie Smith-Bell for her assistance, Dr. Desheng Wang, Dr. Deya Darwish, Roger Bell, Stephen Deci, and Sylwia Mrowka. Also, I would like to thank Dr. Thomas Nelson for his advice troubleshooting some problems and assistance running specificity controls, and Dr. Tapan Khan for his help running western blots. I would like to thank the Blanchette Rockefeller Neurosciences Institute and all its members.

I would like to thank Dr. Lynn Enquist for providing with the transsynaptic viral tracer, PRV essential for this project. Furthermore, I would like to express my gratitude to Dr. Albert Berrebi for providing with the L7 antibody used in this study. In addition, I would like to thank the Behavioral and Biomedical Sciences Training Scholarship for providing funding to purchase some of the materials used in this study. I would like to thank Dr. Silvia Joekes for her guidance performing statistical analyses. In addition, I would like to thank Dustin Long for his assistance using SAS and running statistical analyses. I would like to thank Dr. Karen Martin for her assistance using the Imaging Facility. Finally, I would like to thank the department of Neurobiology and Anatomy and the department of Physiology and Pharmacology at West Virginia University. 


\section{Dedication}

Para mi sobrino Joaquin Nicolas Gonzalez Pellegrini y para todos los que quiero tanto.

In 1873 Camillo Golgi discovered a silver impregnation technique to stain brain tissue that revealed the fine processes of neurons with a level of detail that was unprecedented. The neuroanatomist Santiago Ramón y Cajal employed the Golgi' method to visualize individual neurons and described the structural organization in different regions of the nervous system. In 1906, The Nobel Prize in Physiology or Medicine was jointly awarded to Camillo Golgi and Santiago Ramón y Cajal "in recognition of their work on the structure of the nervous system".

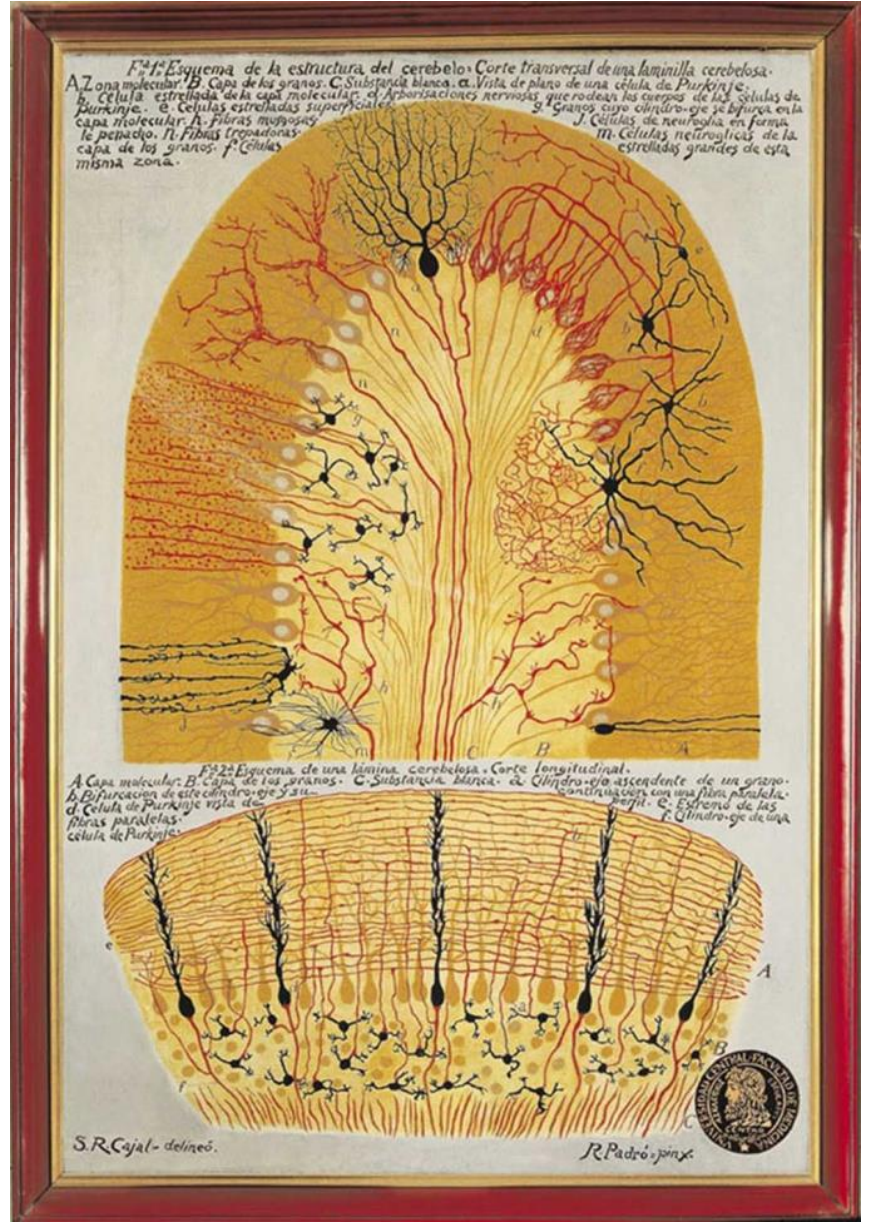

Figure 1. Drawings of the structure of the cerebellum. Drawings of the fine structure of the cerebellum by Santiago Ramón y Cajal, 1894. Painted by R. Padró, 1900.

"Unfortunately, nature seems unaware of our intellectual need for convenience and unity, and very often takes delight in complication and diversity”.

(Ramón y Cajal., 1906 p240). 


\section{Contents}

Title Page

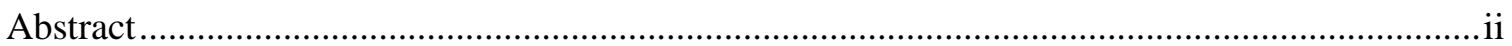

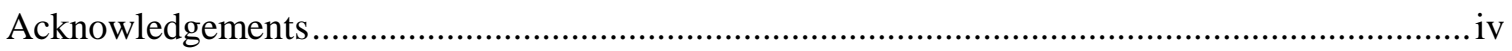

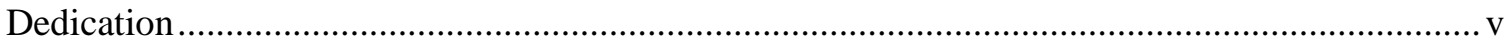

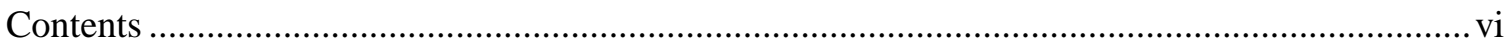

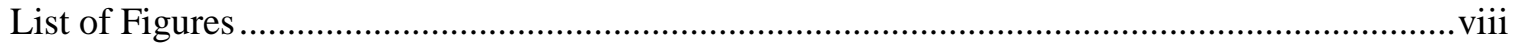

List of Tables ..............................................................................................................

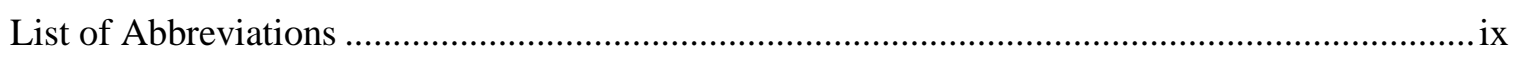

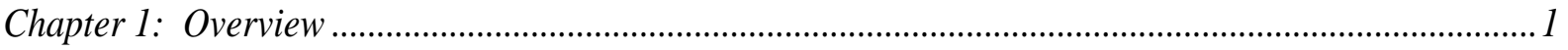

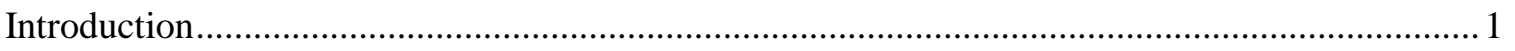

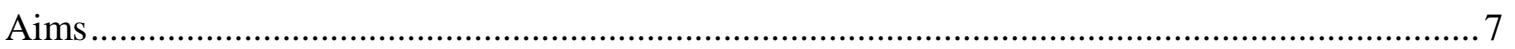

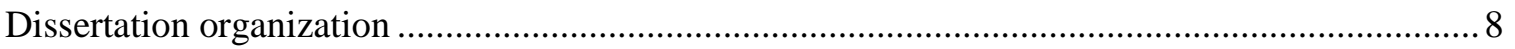

Chapter 2: General Introduction ……………………………………………………... 9

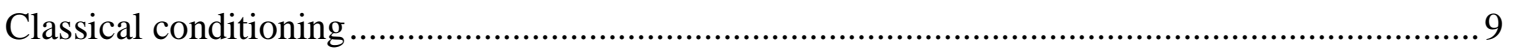

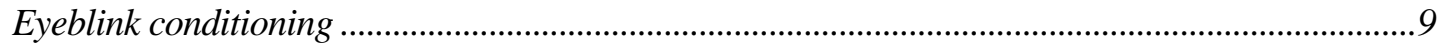

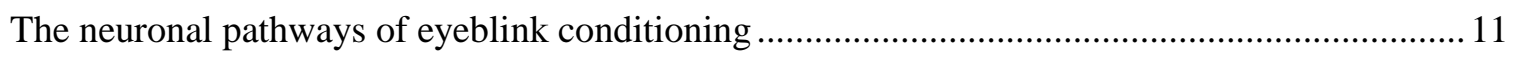

The eyeblink unconditioned response, $U R$........................................................................ 11

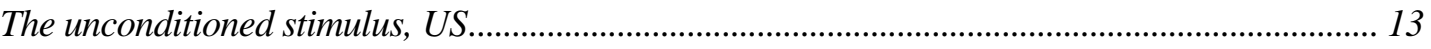

The conditioned stimulus, CS ............................................................................................. 14

The conditioned response, $C R$............................................................................................ 14

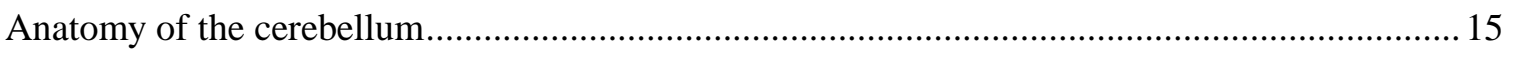

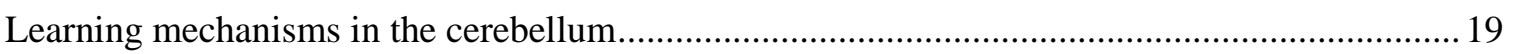

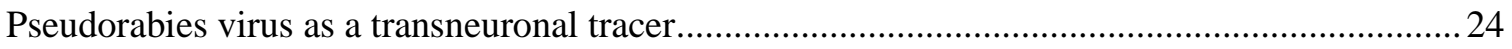

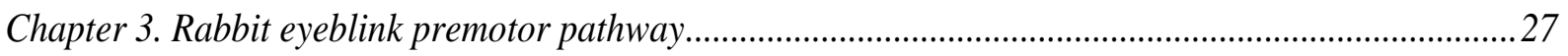

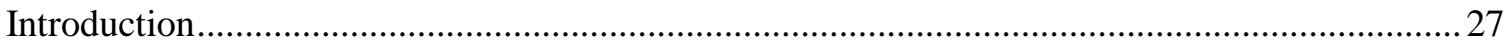

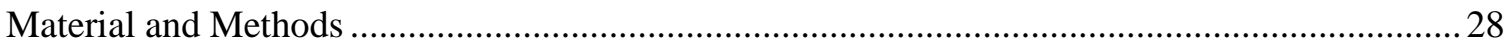

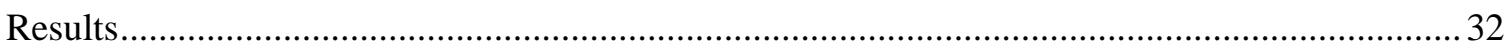

Mapping the OOM premotor pathway …………………………………………………….... 33

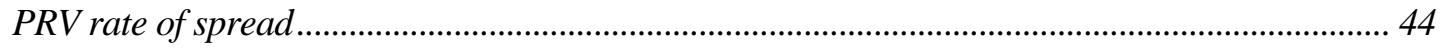

Distribution of premotor neurons in the $D C N$.......................................................................... 44

Neurotransmitter immunoreactivity in the AIN .................................................................... 45

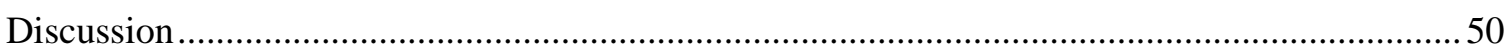

Premotor pathway of the rabbit $\mathrm{OOM}$................................................................................... 51

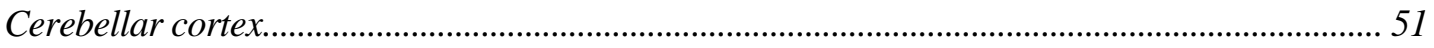

The microcircuit of the AIN …………………………………………………………………..... 52 
Comparison with other transsynaptic studies of the OOM ........................................................... 55

Chapter 4. Synaptic plasticity in eyeblink projection neurons of the AIN .............................................57

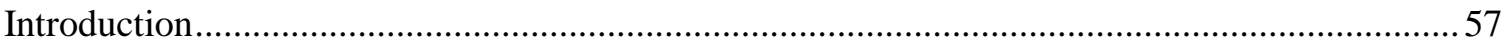

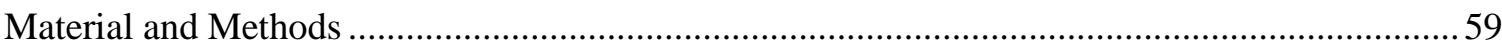

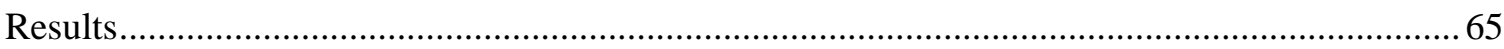

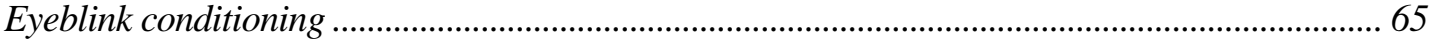

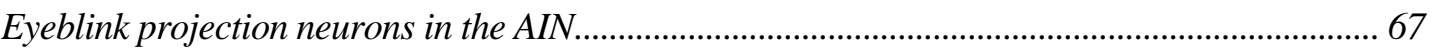

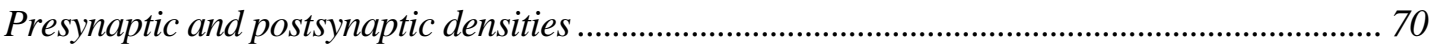

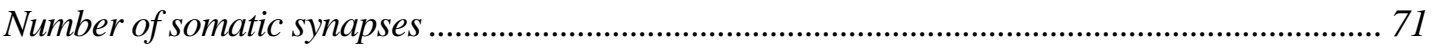

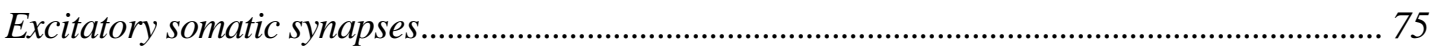

Inhibitory somatic synapses .................................................................................................... 76

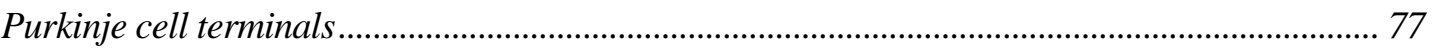

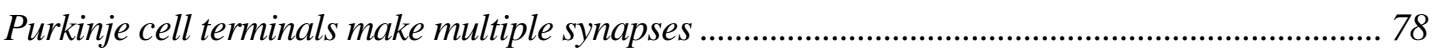

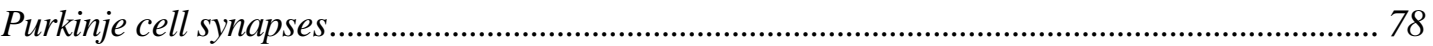

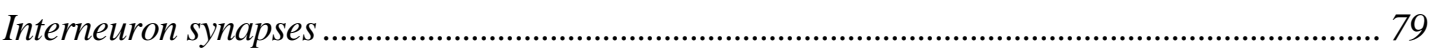

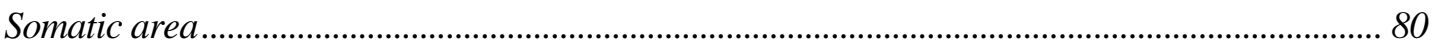

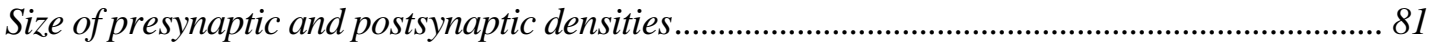

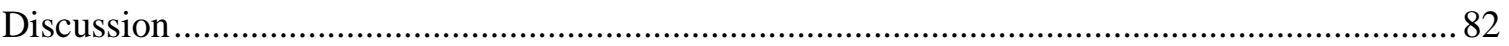

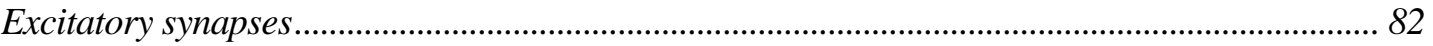

Purkinje cell synapses................................................................................................................ 86

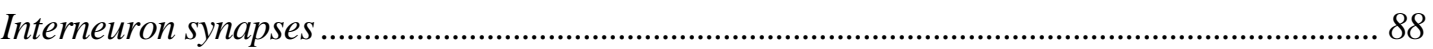

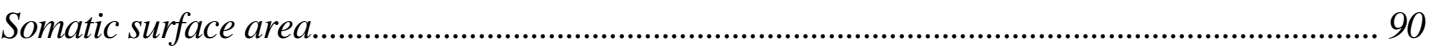

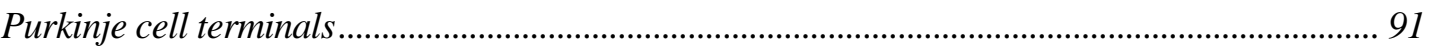

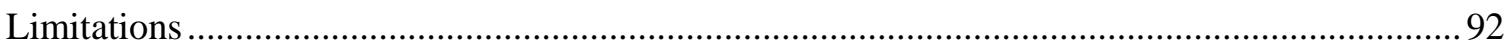

Viability of PRV infected AIN neurons .................................................................................. 92

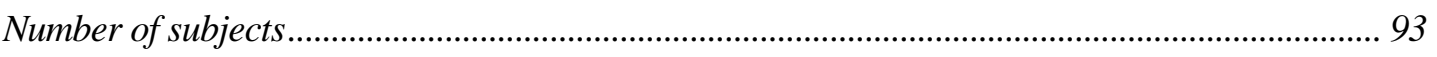

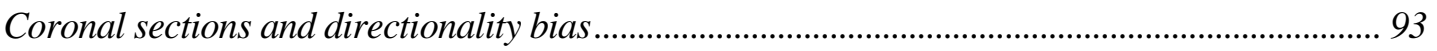

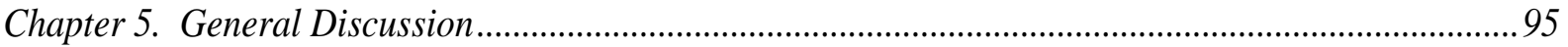

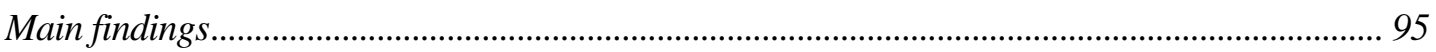

Eyeblink premotor neurons of the anterior interpositus nucleus................................................... 96

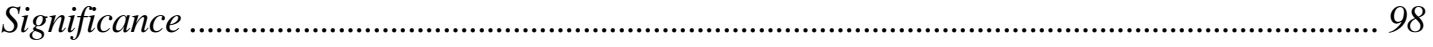

The role of the anterior interpositus in eyeblink conditioning ........................................................ 99

Learning Mechanisms in the Cerebellum..................................................................................... 101

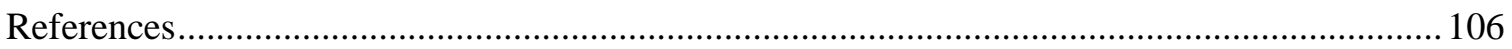




\section{LIST OF FIGURES}

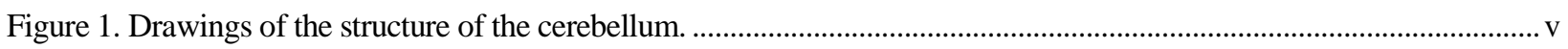

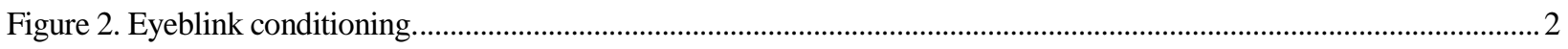

Figure 3. Schematic of the neuronal pathways involved in eyeblink conditioning........................................................... 4

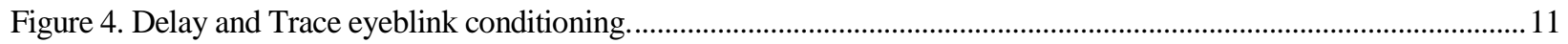

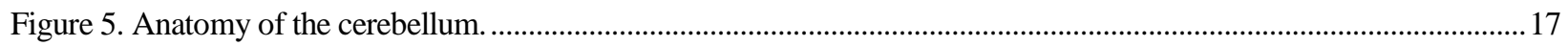

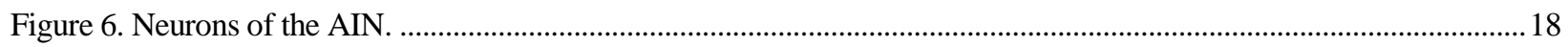

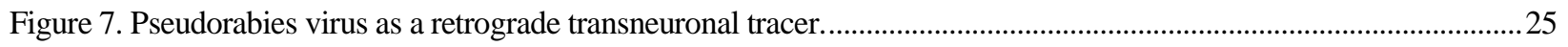

Figure 8. Schematic diagram of the eyeblink premotor pathway labeled with PRV ..........................................................33

Figure 9. Examples of PRV-labeled neurons 3 and 4 days after PRV injection into the OOM..........................................35

Figure 10. Distribution of PRV-labeled neurons 4 days after PRV injection into the OOM. .............................................38

Figure 11. Examples of PRV-labeled premotor neurons $41 \frac{2}{2}$ days after PRV injection into the OOM. ..............................39

Figure 12. Distribution of PRV-labeled neurons 41/2 days after PRV injection into the OOM..........................................41

Figure 13. Examples of PRV-labeled eyeblink premotor neurons 5 days after PRV injection into OOM.........................43

Figure 14. Distribution of PRV-labeled eyeblink premotor neurons in the deep cerebellar nuclei. ...................................45

Figure 15. Glutamate, GABA, and glycine immunoreactivity in the cerebellar cortex.....................................................46

Figure 16. Glutamate, GABA, and glycine immunoreactivity in the deep cerebellar nuclei...........................................47

Figure 17. Eyeblink premotor neuron in the AIN immunoreactive for glutamate..........................................................48

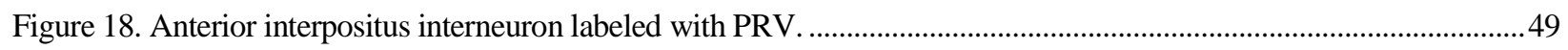

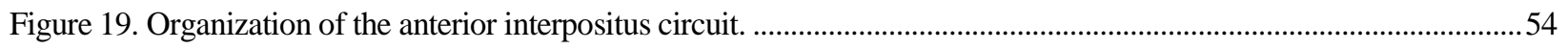

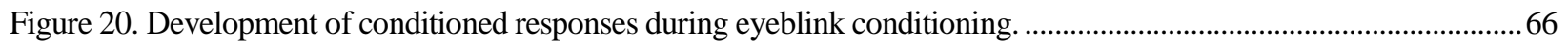

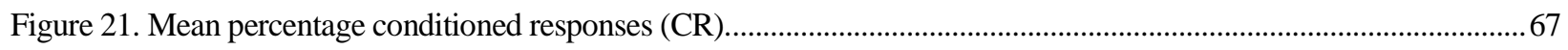

Figure 22. Somatic synapses on identified eyeblink projection neurons of the AIN. …................................................69

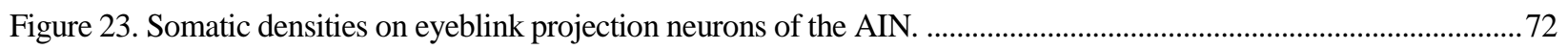

Figure 24. Average number of somatic synapses on eyeblink projection neurons of the AIN. .........................................74

Figure 25. Average number of excitatory somatic synapses on eyeblink projection neurons of the AIN............................75

Figure 26. Number of inhibitory somatic synapses on eyeblink projection neurons of the AIN. ........................................76

Figure 27. Number of Purkinje cell terminals apposing the soma of eyeblink projection neurons of the AIN. ….............77

Figure 28. Number of Purkinje cell somatic synapses on eyeblink projection neurons of the AIN. ..................................79

Figure 29. Number of interneuron somatic synapses on eyeblink projection neurons of the AIN. .....................................8 80

Figure 30. Somatic area $\left(\mu \mathrm{m}^{2}\right)$ of eyeblink projection neurons of the AIN ................................................................... 81

Figure 31. Synaptic plasticity on anterior interpositus eyeblink projection neurons........................................................... 101

Figure 32. Synaptic mechanisms underlying learning in eyeblink conditioning...........................................................103

\section{LIST OF TABLES}

Table 1. Labeled areas after PRV injection into the right orbicularis oculi muscle of the eyelid. .....................................34

Table 2. Somatic synapses on eyeblink projection neurons of the AIN ......................................................................... 73 


\section{LIST OF ABBREVIATIONS}

\begin{tabular}{|c|c|c|c|}
\hline $3 \mathrm{~N}$ & oculomotor nucleus & LTD & long-term depression \\
\hline $4 \mathrm{~N}$ & trochlear nucleus & LTP & long-term potentiation \\
\hline $5 \mathrm{~N}$ & trigeminal nucleus & $\mathrm{m} 5 \mathrm{~N}$ & motor trigeminal nucleus \\
\hline $6 \mathrm{~N}$ & abducens nucleus & $\mathrm{MdD}$ & dorsal medullary reticular nucleus \\
\hline $7 \mathrm{~N}$ & facial nucleus & $\mathrm{MdV}$ & ventral medullary reticular nucleus \\
\hline $12 \mathrm{~N}$ & hypoglossal nucleus & MF & mossy fibers \\
\hline $\operatorname{Acc} 6 \mathrm{~N}$ & accessory abducens nucleus & MG & medial geniculate nucleus \\
\hline AIN & anterior interpositus nucleus & ML & molecular layer \\
\hline AMPAR & $\begin{array}{l}\alpha \text {-amino-3-hydroxy-5-methyl-4- } \\
\text { isoxazolepropionic acid receptor }\end{array}$ & Mve & medial vestibular nucleus \\
\hline $\mathrm{Ca} 2+$ & calcium & NM & nictitating membrane \\
\hline $\mathrm{CF}$ & climbing fiber & NMDAR & N-methyl-D-aspartate \\
\hline $\mathrm{CG}$ & central gray & OOM & orbicularis oculi muscle \\
\hline $\mathrm{Cn}$ & cuneiform nucleus & Op & optic nerve layer of the s. colliculus \\
\hline $\mathrm{CR}$ & conditioned response & PAG & periaqueductal gray \\
\hline Cs & conditioned stimulus & $\mathrm{PaR}$ & pararubral nucleus \\
\hline $\mathrm{CT}$ & control & PB & parabrachial nucleus \\
\hline $\mathrm{DAO}$ & dorsal accessory olive & $\mathrm{PC}$ & Purkinje cell \\
\hline $\mathrm{DC}$ & dorsal cochlear & PCL & Purkinje cell layer \\
\hline $\mathrm{DCN}$ & deep cerebellar nuclei & PD & paired \\
\hline Dk & nucleus of Darkschewitsch & $\mathrm{PF}$ & parallel fibers \\
\hline $\mathrm{dMe}$ & deep mesencephalic nucleus & PIN & posterior interpositus nucleus \\
\hline $\mathrm{DN}$ & dentate nucleus & $\mathrm{PN}$ & pontine nuclei \\
\hline DpG & deep layer of the s. colliculus & $\mathrm{PnO}$ & pontine reticular nucleus, oral \\
\hline $\mathrm{EBC}$ & eyeblink conditioning & $\mathrm{PO}$ & periolivary nucleus \\
\hline EMG & electromyographic & $\operatorname{Pr} 5$ & principal sensory trigeminal nucleus \\
\hline EPSC & excitatory post-synaptic currents & PRV & pseudorabies virus \\
\hline $\mathrm{FN}$ & fastigial nucleus & PSD & postsynaptic density \\
\hline GABA & gamma-Aminobutyric acid & $\mathrm{RN}$ & red nucleus \\
\hline GCL & granule cell layer & RRF & retrorubral field \\
\hline $\mathrm{Gi}$ & gigantocellular reticular nucleus & $\mathrm{SN}$ & substantial nigra \\
\hline $\mathrm{Gr}$ & gracile nucleus & Sol & solitary nucleus \\
\hline HRP & horse-radish peroxidase & $\mathrm{Sp} 5 \mathrm{C}$ & spinal trigeminal nucleus, caudal \\
\hline IC & inferior colliculus & Sp5I & spinal trigeminal nucleus, interpolar \\
\hline IN & interpositus nuclei & $\mathrm{Sp} 5 \mathrm{O}$ & spinal trigeminal nucleus, oral \\
\hline $\mathrm{InC}$ & interstitial nucleus of Cajal & $\mathrm{SpVe}$ & spinal vestibular nucleus \\
\hline InG & int gray layer of the s. colliculus & $\mathrm{SuG}$ & superficial gray layer of the s. colliculus \\
\hline InW & int white layer of the s. colliculus & th & trigeminothalamic tract \\
\hline $\mathrm{IO}$ & inferior olive & $\mathrm{Tz}$ & nucleus of the trapezoid body \\
\hline ir & immunoreactive & UP & unpaired \\
\hline ISI & inter-stimulus interval & UR & unconditioned response \\
\hline KF & Kölliker-fuse nucleus & US & unconditioned stimulus \\
\hline LL & lateral lemniscus & $\mathrm{VC}$ & ventral cochlear nucleus \\
\hline LRt & lateral reticular nucleus & WGA & wheat germ agglutinin \\
\hline
\end{tabular}




\section{Chapter 1: Overview}

\section{Introduction}

One of the main goals in neuroscience is to understand the neuronal mechanisms underlying learning. Learning has been defined as a long lasting change in behavior that results from experience. Behavior is mediated by networks of synaptically connected neurons that are plastic and continuously changing in response to external and internal stimuli. However, exactly where in a network learning takes place depends on the behavioral model. The best models usually involve a simple preparation where the behavior can be easily measured and its neuronal substrates can be studied. This is the case in eyeblink conditioning (EBC), where an eyeblink can be easily measured and the neuronal substrates can be studied using a variety of approaches and techniques. Eyeblink conditioning is a widely used model of associative learning; its neuronal pathways have been well-characterized and they are highly conserved across species.

Eyeblink conditioning is a simple procedure that consists of pairing two stimuli, a conditioned stimulus (CS) and an unconditioned stimulus (US) (Figure 2A, 2B). A tone is frequently used as a CS because it is neutral and does not normally elicit an eyeblink before conditioning (Figure 2A). An airpuff to the cornea is generally used as a US for its ability to reliably produce a reflexive eyeblink or unconditioned response (UR) (Figure 2B). In delay EBC, a tone-CS is presented shortly before an airpuff-US, and the two stimuli then briefly overlap and co-terminate (Figure 2C). After repeated pairings, the organism associates the stimuli and learns to blink to the tone-CS in anticipation of the airpuff-US (Figure 2D). This anticipatory eyeblink is called a conditioned response (CR) and it is regularly used as a measure of learning. Figure 2D shows the early phase of EBC where the acquisition of the first CRs emerge. Figure 2E shows the late phase of EBC as paired training consolidates learning and the CRs gradually shift to peak at about the point where the airpuff-US occurs. Therefore, a behavior such as an eyeblink response is modified by experience and a previously neutral tone-CS becomes capable of eliciting an eyeblink CR. 


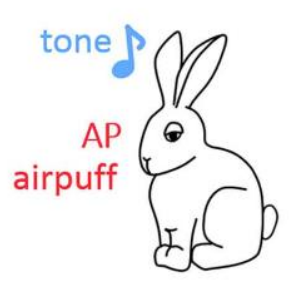

A Conditioned Stimulus

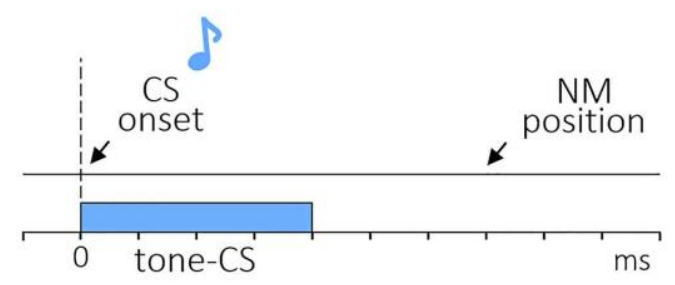

B Unconditioned Stimulus

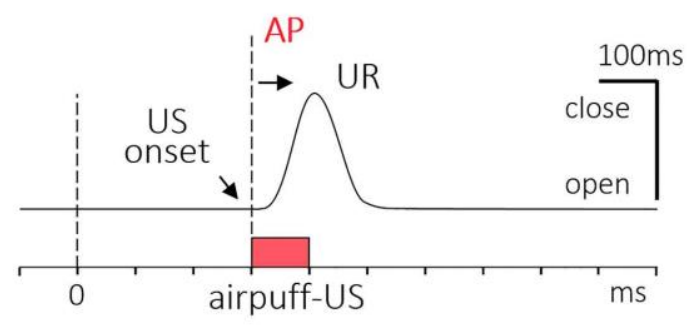

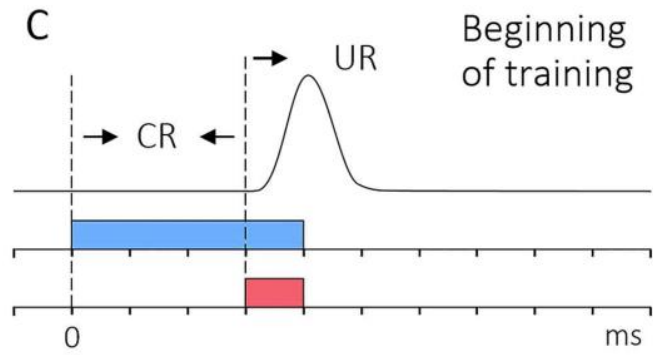
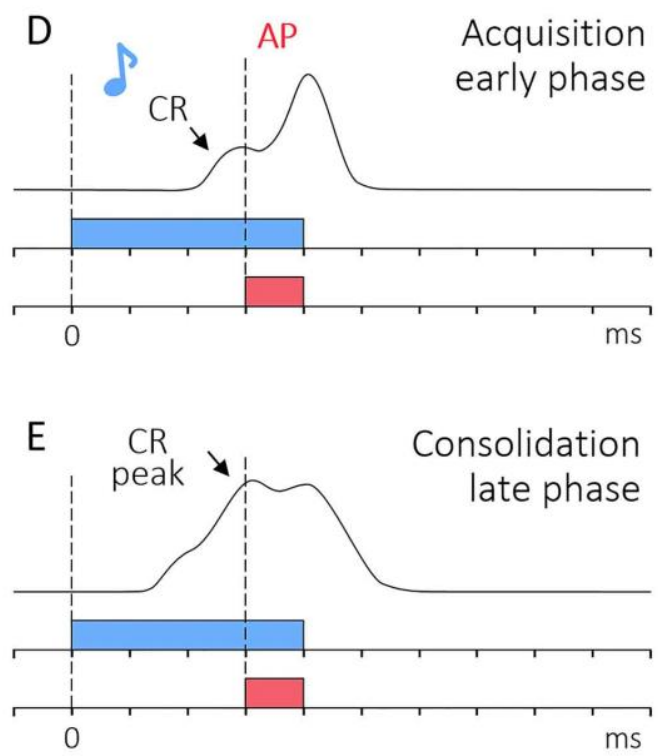

Figure 2. Eyeblink conditioning.

Panel A shows an eyeblink response to a 400ms-tone conditioned stimulus (CS) measured as movement of the nictitating membrane (NM). Panel B shows an eyeblink response to a 100ms-airpuff unconditioned stimulus (US). A reflexive eyeblink unconditioned response (UR) is elicited by the airpuff-US, whereas the tone-CS does not elicit an eyeblink response before training. During delay eyeblink conditioning (EBC) the tone-CS and the airpuff-US are paired together. At the beginning of training, the tone-CS does not elicit an eyeblink response, shown in panel C. However, after repeated pairings, the tone-CS becomes capable of eliciting an anticipatory eyeblink conditioned response (CR). The acquisition of the first CRs during the early phase of EBC is shown in panel D. Prolonged paired training allows for the consolidation of CRs during the late phase of $\mathrm{EBC}$, shown in panel $\mathrm{E}$.

The basic neuronal pathways involved in EBC were identified by recording and lesion studies. In vivo recording studies found multiple regions that fired in a pattern that was correlated with the CS, the US, or the CR. However, lesions of those sites revealed that only a few were 
necessary. Studies in rabbits showed that bilateral removal of the neocortex or hippocampus did not prevent the formation of CRs during delay EBC (Oakley and Russell, 1972; Schmaltz and Theios, 1972; Norman et al., 1974). Furthermore, the acquisition of CRs was possible in decerebrated cats, suggesting that higher order regions anterior to the red nucleus are not essential (Norman et al., 1977). In the early 1980s, Thompson and colleagues found that lesions of the ipsilateral cerebellum abolished the formation and execution of CRs but did not prevent the performance of the eyeblink UR, suggesting that the association between the tone and airpuff takes place in the cerebellum (Lincoln et al., 1982; McCormick et al., 1982b; McCormick and Thompson, 1984a).

A simplified diagram of the major pathways involved in EBC is shown in Figure 3. Auditory information from the tone-CS (shown in blue) is relayed to neurons of the ventral cochlear nucleus, which send projections to the pontine nuclei (PN). Neurons in the PN send projections to the cerebellum in the form of mossy fibers (MF) that contact neurons in the anterior interpositus nucleus (AIN) and granule cells in the cerebellar cortex. The axons of granule cells form parallel fibers (PF) that comprise the major source of synaptic input onto Purkinje cells (PC). Sensory information from the airpuff-US (shown in red) is relayed to neurons of the trigeminal nucleus, which send projections to the inferior olive (IO). Neurons in the IO send projections to the cerebellum in the form of climbing fibers (CF) that contact AIN neurons and PCs. Information about the tone-CS and airpuff-US converge on AIN neurons and on PCs. Purkinje cells are inhibitory, integrate information from PFs and CFs, and form the only output from the cerebellar cortex which is sent to nuclear neurons. Neurons in the AIN integrate excitatory information from MF and CF collaterals, inhibitory information from PCs and local interneurons and form the final output from the cerebellum. The CR (shown in black) is executed by eyeblink projection neurons of the AIN that make excitatory synapses on neurons of the red nucleus $(\mathrm{RN})$ which in turn control motoneurons located in the facial nucleus $(7 \mathrm{~N})$, and other brainstem nuclei that work synergistically to produce a coordinated eyeblink response. 


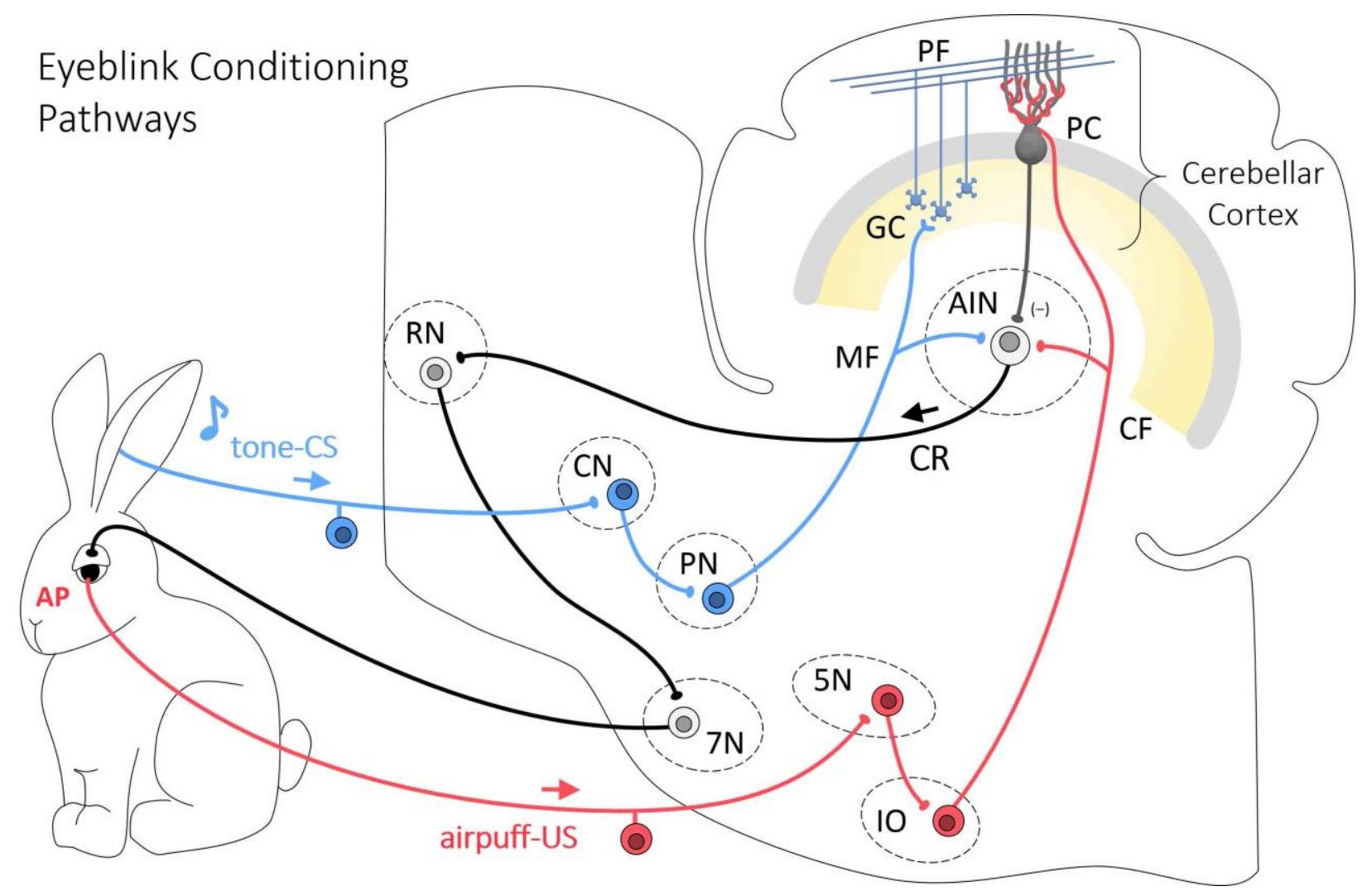

Figure 3. Schematic of the neuronal pathways involved in eyeblink conditioning. A simplified diagram of the minimal circuitry necessary in eyeblink conditioning (EBC). The toneconditioned stimulus (CS) pathway is shown in blue. The airpuff (AP) unconditioned stimulus (US) pathway is shown in red. The conditioned response (CR) pathway is shown in black. Arrows indicate direction of afferent inputs and efferent outputs. Auditory information from the tone-CS is conveyed to the cochlear nucleus $(\mathrm{CN})$ which in turns send projections to the pontine nucleus. Pontine neurons give rise to mossy fibers (MF) that contact neurons in the anterior interpositus nucleus (AIN) and granule cells (GC) in the cerebellar cortex. The axons of GCs form parallel fibers (PF) that contact Purkinje cells (PC). Sensory information from the airpuff-US is conveyed to the trigeminal nucleus $(5 \mathrm{~N})$ which in turn sends projections to the inferior olive (IO). Neurons of the IO give rise to climbing fibers $(\mathrm{CF})$ which contact neurons in the AIN and PCs. Information from the tone-CS and airpuff-US converges in AIN neurons and PCs. In addition, PCs convey information processed in the cerebellar cortex (interneurons are omitted for simplicity) to their target nuclear neurons in the AIN which are the final output from the cerebellum. The CR is executed by AIN neurons that project to the red nucleus $(\mathrm{RN})$ which in turn activates motor neurons in the facial nucleus $(7 \mathrm{~N})$ and other brain stem nuclei that together elicit an eyeblink CR.

Recording and infusion studies identified two regions in the cerebellum as possible sites of plasticity during EBC. The first was found in the dorsolateral AIN of the deep cerebellar nuclei 
and the second was found in PCs of the cerebellar cortex distributed in multiple sites called eyeblink microzones (Clark et al., 1984; Lavond et al., 1984; McCormick and Thompson, 1984a; Hesslow, 1994b; Yeo and Hesslow, 1998; Kotani et al., 2003). However, the firing pattern of PCs did not seem to correlate with the formation or execution of CRs but rather it seemed to encode different features of the CRs (Berthier and Moore, 1986; Hesslow, 1994a; Jirenhed et al., 2007). Information processed in different eyeblink microzones is conveyed to the AIN where it is integrated. The AIN is the ultimate processing center of the cerebellum, where information from PCs and afferent inputs from MFs and CFs converge.

Evidence from lesion, infusion, and recording studies suggests that the engram of learning of EBC may reside in the AIN. Indeed, neurons of the AIN show a firing pattern that is correlated with the formation of eyeblink CRs (McCormick and Thompson, 1984a; Berthier and Moore, 1990; Tracy et al., 2001). In addition, lesion or temporary inactivation of the AIN prevents the acquisition of CRs and also abolishes previous learning (Lavond et al., 1984; Steinmetz et al., 1992; Krupa and Thompson, 1997). Further, temporary inactivation of sites efferent to the AIN can temporarily block the expression but fails to block the formation of eyeblink CRs (Kim and Thompson, 1997). In fact, the AIN is necessary for the association between the tone-CS and airpuff-US, and it is also required for the execution of eyeblink CRs. Because neurons of AIN play a central role in EBC, they are the focus of the current research.

Unlike the cerebellar cortex, the organization of the AIN circuit and its interconnections are not well-understood. The AIN is somatotopically organized. Each side is comprised of $\sim 4,600$ nuclear neurons (in the cat) that are involved in the control and modulation of over $~ 300$ pairs of skeletal muscles (Booth, 1948; Palkovits et al., 1977). The somatotopy of the AIN suggests that a fraction of AIN neurons are involved in eyeblink control and consequently, possible candidates for learning. In addition, even within the eyeblink region of the AIN there are various types of nuclear neurons.

The AIN is a complex structure, comprised of multiple neuronal types that perform different functions. In general, nuclear neurons can be divided into three major types, large glutamatergic neurons that project to the RN, small GABAergic neurons that project to the $\mathrm{IO}$, and small interneurons that project locally. Most of our understanding about the properties of nuclear 
neurons derives from electrophysiological studies conducted on large nuclear neurons, presumed to be glutamatergic projection neurons.

A frequent limitation of electrophysiological studies in vitro, is that distinction among different nuclear neurons can be difficult based solely on their morphological or electrophysiological properties because they often overlap. Another limitation is that the electrophysiological properties of different nuclear neurons are frequently hard to relate to their function. On the other hand, single or multi unit recording studies in vivo encounter the opposite challenge. While neuronal activity can be related to function, the identity of those neurons is usually unknown. Despite its importance, the role of the different AIN neurons and their function during EBC remain uncertain.

The main focus of this Dissertation was to study the possible mechanisms of learning in the AIN during EBC. There are two major challenges for the study of learning in the AIN during EBC. First, the identity of different neuronal types and their role during EBC are unknown. Second, a functional relationship between specific nuclear neurons and the eyeblink response is difficult to establish.

Because different types of nuclear neurons perform different tasks during EBC, they are probably susceptible to different or even opposite changes during learning. For this reason, the identity of nuclear neurons is a pre-requisite to make meaningful interpretations about learningspecific changes taking place during EBC.

Evidence suggests that learning during EBC is mediated by synapse formation. Interestingly, the distribution of synapses differs between large and small nuclear neurons. Whereas small nuclear neurons receive only sparse somatic afferents, large nuclear neurons are covered by afferent inputs. Because they can directly influence signal output, changes in somatic synapses can have a greater impact on neuronal function. For this reason, learning during EBC may be mediated by changes in somatic synapses of large eyeblink projection neurons of the AIN. 


\section{Aims}

The major goal of this Dissertation was to identify AIN neurons involved in the eyeblink premotor pathway and examine possible changes in their synaptic inputs as a function of EBC. In order to identify eyeblink projection neurons of the AIN, a retrograde transsynaptic viral tracer, pseudorabies virus (PRV) was injected into the eyelid (orbicularis oculi muscle) of the rabbit.

\section{Aim 1: Identify the eyeblink premotor pathway of the rabbit.}

A complete delineation of the eyeblink premotor pathway was accomplished by following the progression of PRV at different time intervals. Next, eyeblink premotor neurons of the AIN were further characterized based on their neurotransmitter content to classify the different neuronal types involved in the eyeblink pathway.

\section{Aim 2: Characterize the different types of eyeblink premotor neurons of the AIN based on their neurotransmitter content.}

An eyeblink premotor network comprised of projection neurons and local interneurons was characterized. In order to determine a possible mechanism underlying learning, the effect of $\mathrm{EBC}$ on the number of excitatory and inhibitory somatic synapses of eyeblink projection neurons was examined.

\section{Aim 3: Quantify changes in the number of excitatory and inhibitory somatic synapses of eyeblink projection neurons as a function of learning.}

Changes in the number of excitatory and inhibitory somatic synapses of eyeblink projection neurons in rabbits that underwent different behavioral procedures were observed. Finally, a possible mechanism underlying learning in the AIN was described. 


\section{Dissertation organization}

This Dissertation is organized in 5 Chapters. First, an overview of the main concepts and the framework that led to the development of the specific Aims is elaborated in Chapter 1. Next, a comprehensive review of the literature and background is expanded in Chapter 2. Experiment 1 examined specific Aim 1 and Aim 2 and is covered in Chapter 3. Specific Aim 3 was investigated in Experiment 2 and is described in Chapter 4. Last, in Chapter 5 the main findings and the functional significance of this study are discussed. 


\section{Chapter 2: General Introduction}

\section{Classical conditioning}

Eyeblink conditioning belongs to a broader type of associative learning called classical or Pavlovian conditioning. Classical conditioning was first described by the Russian physiologist Ivan Pavlov at the end of the 1800s while he was studying the digestive processes in dogs that earned him the Nobel Prize in 1904. Pavlov noticed a peculiar behavior; while food stimulates a salivatory reflex, he noticed that dogs began to salivate when the keeper bringing their food entered the room. Based on this observation, Pavlov decided to investigate this response using a controlled and systematic approach where the sound of a ticking metronome was presented first and the food was administered shortly after. After several presentations of this sequence of sound followed by food, Pavlov noticed that the dogs began to salivate when they heard the metronome and before receiving the food. "A stimulus which was neutral in and of itself had been superimposed upon the action of the inborn alimentary reflex," Pavlov wrote in his results (p. 27). "We observed that, after several repetitions of the combined stimulation, the sounds of the metronome had acquired the property of stimulating salivary secretion" (Pavlov, 1927). Classical conditioning can be implemented in a variety of visceral and musculoskeletal responses and some of the most common include: eyeblink, heart rate, taste aversion, limb flexion, vestibular ocular reflex, and fear conditioning.

\section{Eyeblink conditioning}

Eyeblink conditioning was first implemented in human subjects at the beginning of the 1900s and proved to be an invaluable tool for studying procedural and declarative learning (WoodruffPak, 2000). Eyeblink conditioning is a simple preparation that has been used in a wide range of species including, rabbits, cats, rats, mice, ferrets, guinea pig, dogs, and monkeys to name a few. However, most of our understanding about the neuronal substrates involved in EBC come from the rabbit model, first described by Gormezano and colleagues in 1962 (Gormezano et al., 1962). Rabbits became the subject of choice for the study of EBC for several reasons. Unlike 
other species, rabbits tolerate being restrained for long periods without moving thus permitting accurate measurement of the eyeblink response with minimal instrumentation. In addition, they have a base rate of spontaneous blinking that is well below most mammals and chances of spontaneous blinks during the CR period are rare. Another reason is that unlike other species that usually show an orientating or alpha response to CS presentations that can complicate behavioral measurements (Figure 4A), rabbits do not show an orientating response to most CSs used in EBC including the commonly used tone and light CS (Cegavske and Thompson, 1976; Disterhoft et al., 1985; Gruart et al., 2000; Leal-Campanario et al., 2004b).

There are a number of factors that can affect the rate of $\mathrm{CR}$ acquisition during EBC. One important factor is the sequence of CS and US presentations. As a general rule, the CS has to precede the US and they have to occur in temporal proximity (contiguity) for an association to occur (Lashley and Wade, 1946). Experiments where the CS was presented simultaneously with the US were unable to produce CRs and experiments that presented the CS after the US (backward conditioning) were also incapable of inducing CRs (Smith et al., 1969). In general, conditioning is acquired more rapidly if the CS onset precedes the US onset, and the two stimuli briefly overlap and co-terminate. This preparation is also known as delay conditioning (Figure 4C). On the other hand, conditioning is acquired more slowly if a gap (trace) is introduced between the CS and US. This preparation is also known as trace conditioning (Figure 4D). Another important factor is the inter-stimulus interval (ISI) which refers to the time period between the CS onset and the US onset. The optimal ISI induces rapid conditioning and as the ISI becomes longer, conditioning becomes more difficult. The optimal ISI in EBC is 200400ms (Smith et al., 1969). In addition to the sequence of CS and US presentations, a number of other factors are also important and can affect the rate of conditioning including the intensity of the stimuli, especially the intensity of the US, the duration of the stimuli, the duration of the interval between trials and of course, the number of training sessions (Moore, 2002). 
Conditioned Stimulus

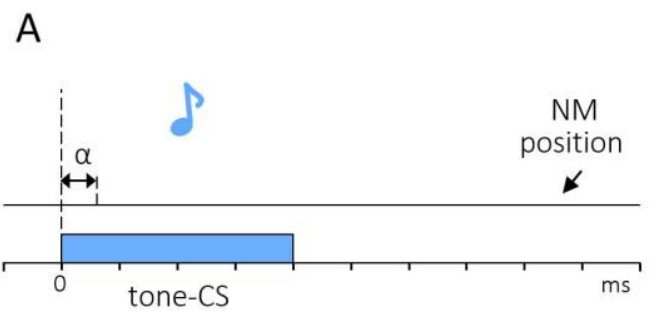

Delay Eyeblink Conditioning

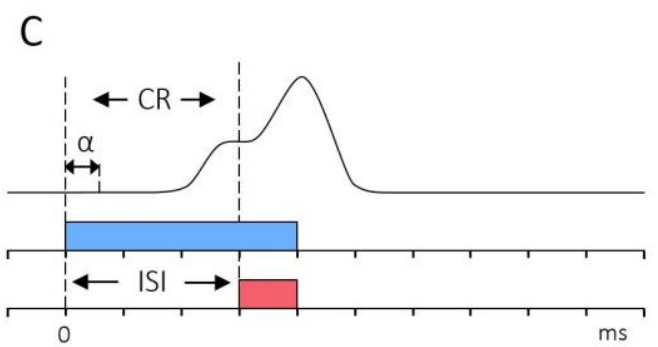

Unconditioned Stimulus

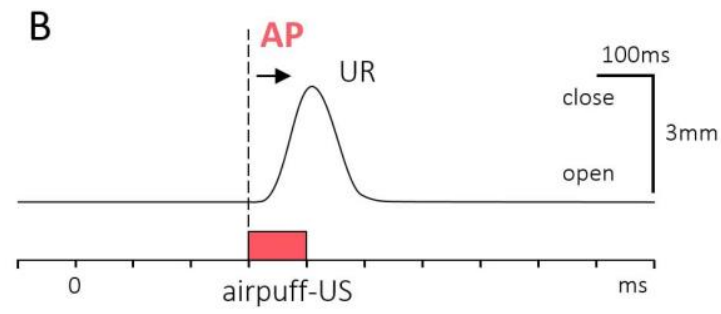

Trace Eyeblink Conditioning

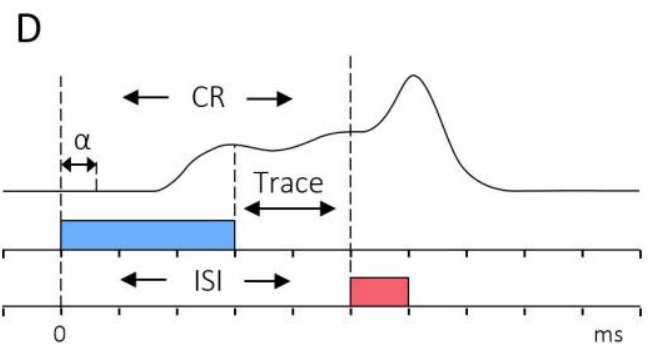

Figure 4. Delay and Trace eyeblink conditioning.

Eyeblink responses measured as movement of the nictitating membrane (NM). A, a tone (shown in blue) is commonly used as a conditioned stimulus (CS), and in the rabbit a tone rarely elicits an alpha $(\alpha)$ response. B, an airpuff (shown in red) to the cornea is commonly used as an unconditioned stimulus (US) for its ability to reliably elicit a reflexive eyeblink unconditioned response (UR). C, Delay eyeblink conditioning (EBC). A $400 \mathrm{~ms}$ tone-CS is paired with a $100 \mathrm{~ms}$ airpuff-US. The tone-CS is presented first and is shortly followed by the airpuff-US, the two stimuli then briefly overlap and coterminate. The inter-stimulus interval (ISI) refers to the period between the CS and US onset and in this case is 300ms. D, in Trace EBC a gap or trace is introduced between the CS offset and the US onset. The ISI in this case is 500ms. Delay and Trace EBC are cerebellar-dependent. However, in addition to the cerebellum, other brain regions are also necessary in Trace EBC. C and D, After repeated pairings of the tone-CS and airpuff-US an association between the stimuli is formed and the tone becomes capable of eliciting an anticipatory eyeblink conditioned response (CR).

\section{The neuronal pathways of eyeblink conditioning}

\section{The eyeblink unconditioned response, $U R$}

In the rabbit, an eyeblink is a coordinated response that involves the activation and inhibition of three muscles that work synergistically (Cegavske and Thompson, 1976; Cegavske et al., 1979; 
Berthier, 1984; Disterhoft et al., 1985; Berthier, 1992). Activation of the Orbicularis Oculi muscle $(O O M)$, and inhibition of the tonically active levator palpebrae muscle allow the eyelids to contract, while simultaneous activation of the retractor bulbi muscle pulls the eyeball into the eye socket and allows the passive movement of the nictitating membrane (NM) across the cornea (Gormezano et al., 1962; Schneiderman et al., 1962; Deaux and Gormezano, 1963; McCormick et al., 1982a). Motoneurons activating these muscles are located in different brainstem motor nuclei. Motoneurons innervating the $O O M$ are located in the dorsolateral portion of the ipsilateral facial nucleus (7N) (Radpour, 1977; Komiyama et al., 1984; Furutani and Sugita, 2008). Motoneurons controlling the levator palpebrae muscle are located in the contralateral oculomotor nucleus (3N) (van Ham and Yeo, 1996b; May et al., 2012). Motoneurons controlling the retractor bulbi muscle are located in the ipsilateral accessory abducens nucleus (acc6N) and to a lesser extent in the abducens nucleus (6N) (Gray et al., 1981; Berthier and Moore, 1983; Disterhoft et al., 1985).

A reflexive eyeblink can be elicited using a periocular stimulus such as an airpuff that activates sensory neurons that project to the trigeminal nucleus (5N) (Hiraoka and Shimamura, 1977; Harvey et al., 1984; van Ham and Yeo, 1996b; Henriquez and Evinger, 2007). Trigeminal neurons activate motoneurons in the ipsilateral $7 \mathrm{~N}$ and acc6N, while inhibiting motoneurons in the contralateral $3 \mathrm{~N}$ to produce an eyeblink response (Hiraoka and Shimamura, 1977; McCormick et al., 1982a; Pellegrini et al., 1995; van Ham and Yeo, 1996b; Delgado-Garcia et al., 2003; May et al., 2012).

Together, an eyeblink is a synchronized response that involves the activation of the $O O M$ and the retractor bulbi muscle and the simultaneous inhibition of the levator palpebrae muscle by motoneurons located in the $7 \mathrm{~N}$, acc6N, and $3 \mathrm{~N}$ respectively. Lesion of any of these muscles or motor nuclei impairs the eyeblink response. Eyeblinks can be measured in a variety of ways including transduction of the NM, recording eyelid movement or $O O M$ electromyographic (EMG). Eyeblink responses measured with these methods are highly correlated (Berthier, 1992; Leal-Campanario et al., 2004a). In the rabbit, movement of the nictitating membrane is commonly preferred because of its simplicity and minimal invasiveness compared to other methods (Gormezano et al., 1962). 


\section{The unconditioned stimulus, $U S$}

Neurons in the sensory trigeminal nuclei that receive sensory afferents from the cornea airpuff project to the contralateral IO and contact neurons located in a region known as the dorsal accessory olive (DAO) (Mauk et al., 1986). The IO receives somatosensory, proprioceptive, motor, vestibular, and visual afferents from the $5 \mathrm{~N}$, spinal cord, dorsal column, $\mathrm{RN}$, superior colliculus, and other nuclei in the mesodiencephalic junction (Boesten and Voogd, 1975; Brown et al., 1977; Berkley and Hand, 1978; Swenson and Castro, 1983; Onodera, 1984; Holstege and Tan, 1988; Akaike, 1992; Molinari et al., 1996). As mentioned earlier, the IO is the only source of $\mathrm{CF}$ inputs to the cerebellum. Axons from the IO project to the contralateral cerebellum through the inferior peduncle and contact neurons in the DCN, by way of CF collaterals, and PC in the cerebellar cortex (Armstrong, 1974; Brodal et al., 1975; Tolbert et al., 1976; Van der Want et al., 1989; Ruigrok and Voogd, 2000; Pijpers et al., 2005). Each PC is innervated by a single $\mathrm{CF}$, however a single IO axon gives rise to multiple $\mathrm{CFs}$ that contact $\sim 7$ different PCs (in the rat) (Schild, 1970; Delhaye-Bouchaud et al., 1985; Shinoda et al., 2000; Sugihara et al., 2001). Climbing fibers wrap themselves around the proximal dendrites of their target PCs and form powerful synaptic contacts capable of eliciting a strong and multiphasic complex spike. The airpuff-US elicits $\sim 2$ action potentials in DAO neurons that in turn consistently produces a complex spike in the postsynaptic PC (Campbell and Hesslow, 1986b, a). Conversely, the IO receives feedback inhibitory from GABAergic projection neurons of the DCN (Andersson et al., 1988). During EBC GABAergic projection neurons from the AIN inhibit the DAO particularly during the execution of CRs (Sears and Steinmetz, 1991). Lesion or inactivation of the DAO prevents the formation of CRs when made before training and results in extinction or complete abolition of CRs when made after training (McCormick et al., 1985; Voneida et al., 1990). Indeed, inactivation of the IO produces an increase in the firing rate of PCs, which in turn inhibits nuclear output and disrupts cerebellar function (Montarolo et al., 1982; Benedetti et al., 1983; Batini and Billard, 1985). Nevertheless, the airpuff-US activates neurons in the DAO that project to the cerebellum by way of CFs that contact AIN and PCs. Electrical stimulation of the DAO can be used as an effective US and can produce more rapid acquisition of CRs than its counter part airpuff-US when paired with a CS (Mauk et al., 1986; Steinmetz et al., 1989). 


\section{The conditioned stimulus, $C S$}

Auditory information from the tone-CS is relayed to neurons of the ventral cochlear nucleus, which send projections to the basolateral PN (Steinmetz et al., 1987). The PN receive afferent inputs from various brainstem nuclei and from the somatosensory and association areas of the cerebral cortex (Brodal and Jansen, 1946; Enger and Brodal, 1985). The PN is one of the major sources of MF inputs to the cerebellum and send projections through the middle cerebellar peduncle. Mossy fibers contact neurons in the AIN and granule cells in the cerebellar cortex which in turn contact PCs by way of PFs. Activation of PFs elicit simple spikes on their postsynaptic PCs. Lesioning the middle cerebellar peduncle prevents acquisition and immediately abolishes retention of the eyelid CR to all modalities of CSs such as tone-CS and light-CS. Whereas lesioning the basolateral PN can selectively extinguish the eyelid CR to a tone-CS while the CRs to a light-CS remains intact (Steinmetz et al., 1986b; Lewis et al., 1987; Rosen et al., 1989). On the other hand, electrical stimulation of the PN can be used as a CS, and it can induce CRs more rapidly than a tone-CS (Steinmetz et al., 1986a; Lavond et al., 1987).

\section{The conditioned response, $C R$}

The essential efferent CR pathway consists of projection fibers exiting from the AIN ipsilateral to the trained eye through the superior cerebellar peduncle, crossing to innervate the contralateral $\mathrm{RN}$ and ultimately activating the $O O M$ and retractor bulbi muscle on the ipsilateral side while inhibiting the levator palpebrae muscle to generate a precisely timed and coordinated eyeblink response (Thompson, 1988). Early tracing studies using HRP and WGA showed direct projections from the interpositus nucleus to the contralateral $\mathrm{RN}$ through the superior cerebellar peduncle (Flumerfelt, 1978). Interpositus projection neurons are immunoreactive for glutamate and produce excitatory post-synaptic currents (EPSC) on their postsynaptic RN target neurons (Eccles et al., 1975; Fanardjian et al., 1987; Desmond and Moore, 1991; Pacheco-Calderon et al., 2012).

The RN occupies a strategic position in the brainstem, receiving descending motor commands from the cerebellum and cerebral cortex, and projecting to a variety of motor and sensory nuclei in the brainstem. During EBC the RN acts as a relay nucleus for motor commands generated in 
the AIN. Neuronal recordings from dorsolateral RN and neighboring pararubral nucleus identified a group of eyeblink premotor neurons that are antidromically activated by motoneurons in the 7N and acc6N (Grant and Horcholle-Bossavit, 1986; Holstege and Tan, 1988). When stimulated, these RN neurons generate eyeblink responses. More importantly, inactivation of the $\mathrm{RN}$ before $\mathrm{EBC}$ does not prevent the formation of CRs. Even though the expression of CRs is blocked, the formation of CRs is unaffected and once the RN inactivation is lifted the expression of CR resumes normally. In addition, inactivation of the RN in well-trained animals blocks the expression of the eyeblink CRs while the learning-induced CRs in the AIN are unaffected (Rosenfield and Moore, 1985; Grant and Horcholle-Bossavit, 1986; Haley et al., 1988; Krupa et al., 1993; Krupa and Thompson, 1995; Robleto and Thompson, 2008). Firing rates of these RN and pararubral neurons suggests that they were involved in the performance of CRs rather than in the acquisition of CRs (Chapman et al., 1988; Desmond and Moore, 1991; Porras-Garcia et al., 2010).

\section{Anatomy of the cerebellum}

The cerebellum is located posterior to the cerebrum and dorsal to the pons and brainstem; connected to the rest of the brain by afferent and efferent fibers bundles that form the superior, middle, and inferior cerebellar peduncles. Unlike the cerebrum that has a contralateral representation of the body, the somatotopy of cerebellum is ipsilateral. The cerebellum is comprised of two regions, the deep cerebellar nuclei (DCN) at the core and the cerebellar cortex on the surface (Figure 5). The DCN are divided into three anatomical and functional distinct nuclei: the fastigial (FN), interpositus (IN), and dentate nuclei (DN). The IN is further divided into the anterior interpositus, AIN and posterior interpositus nucleus (PIN), shown in Figure 5D. The cerebellar cortex is divided into three mediolateral regions on each side, the vermis, the paravermis or intermediate hemisphere, and the lateral hemisphere. Each of these regions is folded into lobules (I-X) and each lobule is further divided into folia. The cerebellar cortex is an homogeneous structure, comprised of few neuronal types arranged in a repetitive pattern and can be divided into three layers, shown in Figure 5E (Ramón y Cajal, 1894). The superficial molecular layer (ML) contains inhibitory interneurons (stellate, basket, and Lugaro cells), the 
middle Purkinje cell layer (PCL) is formed by the cell bodies of PCs, and the deep granule cell layer (GCL) is comprised by granule cells, and inhibitory interneurons (Golgi cells) (Figure 5E). The connectivity between the cerebellar cortex and the DCN is highly organized. For the most part, the FN receives cortical inputs from the vermis and projects to vestibular and reticular nuclei, the AIN and PIN receive cortical inputs from intermediate hemispheres project to the contralateral $\mathrm{RN}$, and the $\mathrm{DN}$ receives cortical inputs from the lateral hemispheres and projects to the contralateral RN and thalamus.

The uniform organization of the cerebellar circuit suggests that all regions of the cerebellum perform identical operations. Therefore, the function of specific regions is determined by the source of its afferent inputs and most importantly by the target of its efferent outputs. There are two major sources of afferent inputs to the cerebellum, MFs and CFs. Mossy fibers originate from multiple nuclei in the pons and brainstem. The pattern of MF projections to the cerebellar cortex forms a "patchy" mosaic representation in multiple locations (Voogd and Ruigrok, 1997; Voogd and Glickstein, 1998). Climbing fibers, on the other hand, originate exclusively from the IO. The IO is highly organized structure, comprised of multiple subnuclei that send projections to distinct cortical and nuclear regions. The pattern of CF projections divides the cerebellar cortex into longitudinal zones (A, B, C1, C2, C3, D1, and D2) which are highly conserved across species (Brochu et al., 1990; Sanchez et al., 2002; Sillitoe et al., 2005; Apps and Hawkes, 2009). Longitudinal zones are comprised of an array of PCs organized in sagittal bands that receive CF inputs from the same IO subnucleus (olivo-cortical projection), and share the same nuclear target (cortico-nuclear projection). In addition, cortico-nuclear and olivo-nuclear projections from $\mathrm{CF}$ collaterals converge on the same nuclear region, which in turn send inhibitory projections to the IO forming a reciprocal olivo-cortico-nuclear loop (Hesslow, 1994a; Apps, 1999; Pijpers et al., 2005; Sugihara and Quy, 2007; Sugihara and Shinoda, 2007; Apps and Hawkes, 2009). Within longitudinal zones there are specialized microzones that receive information from CF fields with a particular somatic or sensory modality. The representation of a specific body part is repeated in multiple locations or microzones (fracture somatotopy), and together, they form a functional module or microcomplex (Cerminara and Apps, 2011; Ruigrok, 2011). 

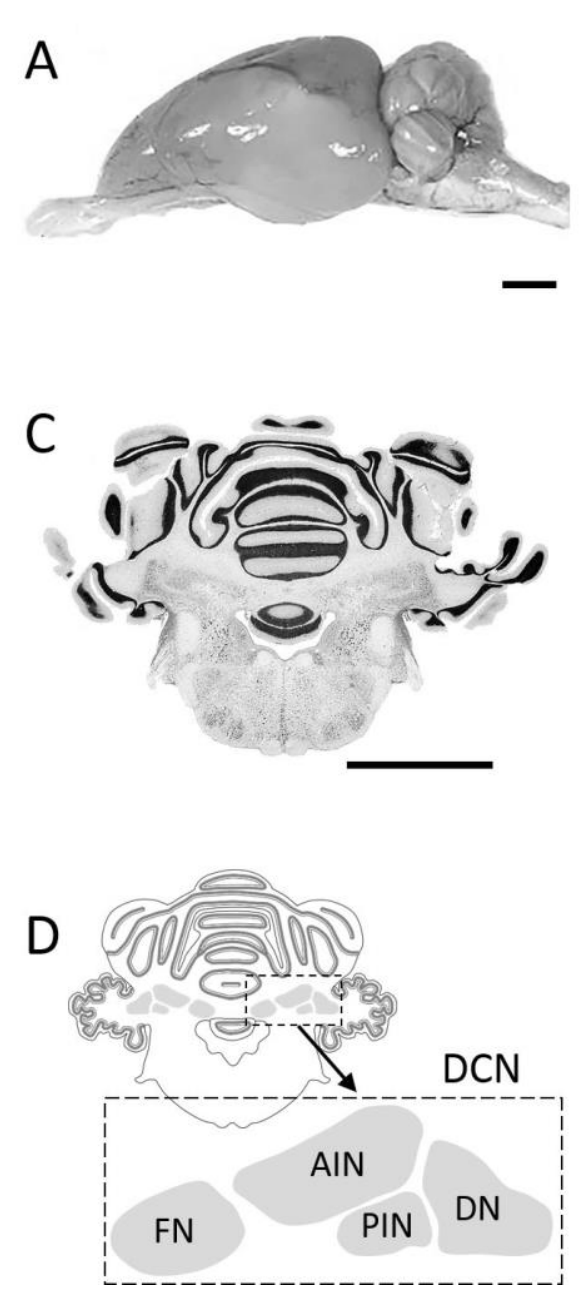
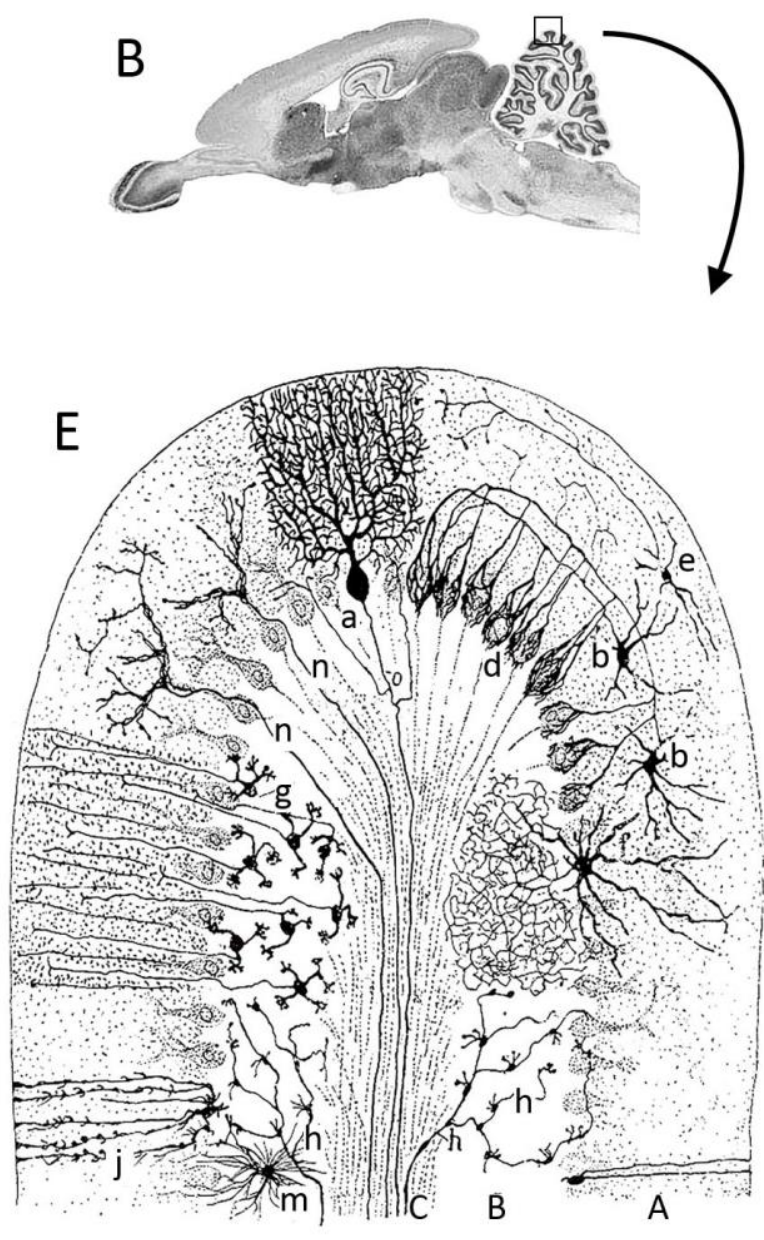

Figure 5. Anatomy of the cerebellum.

A, lateral view of the rabbit brain. B, sagittal section of the rabbit brain. C, coronal section of the rabbit brain. D, schematic of a coronal section showing the deep cerebellar nuclei (DCN) comprised of the fastigial nucleus (FN), the anterior interpositus nucleus (AIN), the posterior interpositus nucleus (PIN), and the dentate nuleus (DN). E, schematic of the different neurons comprising the cerebellar cortex, adapted from (Ramón y Cajal, 1888). A, molecular layer; B, granule layer; C, white matter; a, Purkinje cell; b, basket cell; d, basket cell arborizations surrounding Purkinje cells; e, stellate cells; f, Golgi cells; g, granule cells; h mossy fibers; $\mathrm{j}$ and m, neuroglia; $\mathrm{n}$, climbing fibers. Scale bar 5mm.

The anatomy of the DCN, on the other hand is not as clear. The DCN is comprised of $\sim 30,000$ neurons in the mouse and 46,000 in the cat (Palkovits et al., 1977; Sultan et al., 2002). Initial classification of the nuclear neurons was based on morphology and identified more than six different types (Chan-Palay, 1973a). More recent classifications are based on connectivity, size, 
and neurotransmitter content (Chen and Hillman, 1993; De Zeeuw and Berrebi, 1995; Kleim et al., 2002; Uusisaari et al., 2007). Based on these classifications, nuclear neurons can be separated into three main types (Figure 6). The first type are large glutamatergic neurons that project to the RN and other extracerebellar sites and comprise about $\sim 50-60 \%$ of the total population (Batini et al., 1992; Teune et al., 1995; Teune et al., 2000). The second type are small GABAergic neurons that project to the IO forming the nucleo-olivary loop and constitute $\sim 30 \%$ (Tolbert et al., 1976; De Zeeuw et al., 1989). The third type are inhibitory interneurons which arborize locally and comprise $~ 20 \%$ (Chan-Palay, 1973b; Czubayko et al., 2001; Aizenman et al., 2003). All of these neurons are heterogeneously distributed through the DCN.

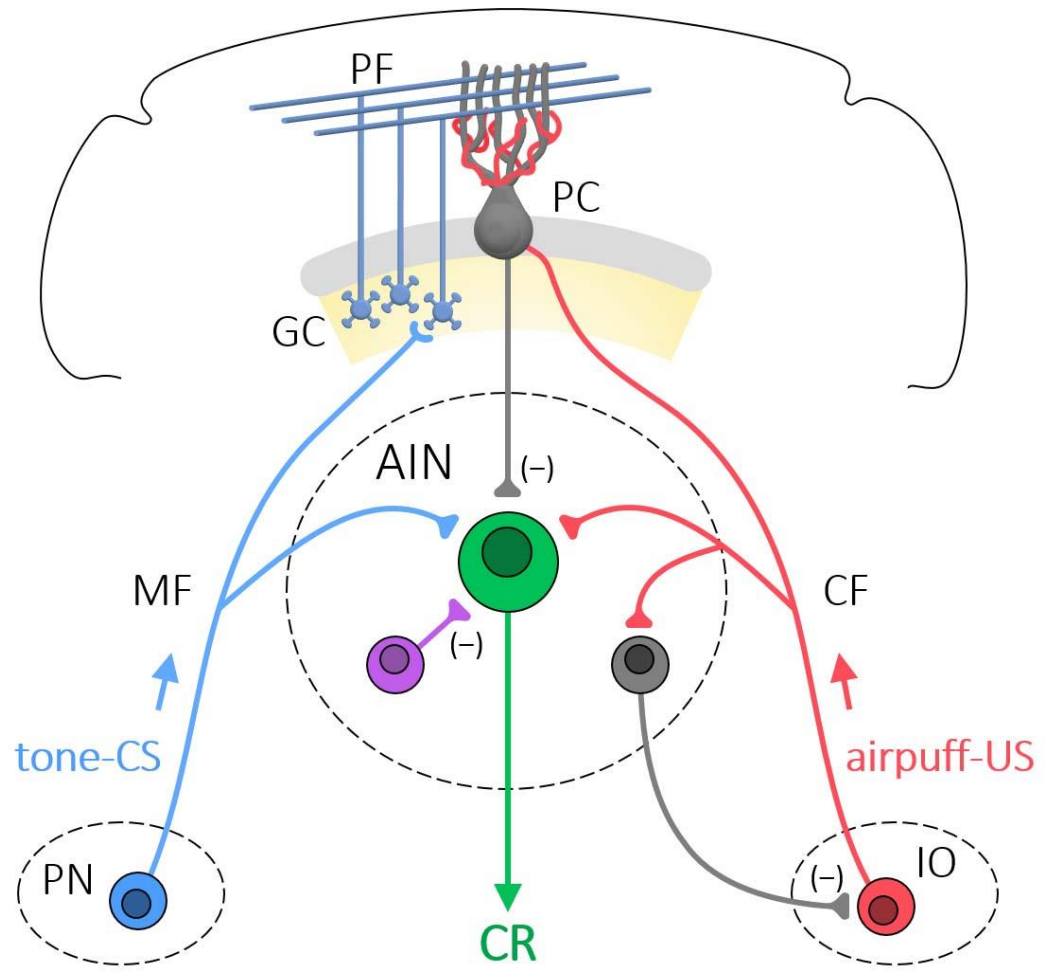

Figure 6. Neurons of the AIN.

Schematic representation of the neurons of the anterior interpositus nucleus (AIN). A large excitatory projection neuron is shown in green, a small GABAergic neuron projecting to the inferior olive (IO) is shown in gray, a local interneuron is shown in purple. 
Nuclear neurons receive a considerable degree of convergence from extracerebellar and intracerebellar afferent fibers. The vast majority of extracerebellar afferent inputs originate from excitatory MF collaterals and to a smaller degree from CF collaterals (Brodal and Jansen, 1946; Eager, 1968; Matsushita and Iwahori, 1971a; O'Leary et al., 1972; Angaut and Sotelo, 1973; Chan-Palay, 1973d, b). There is also a small and sparse source of amine afferents that constitute about $2 \%$ of the synaptic input of nuclear cells (Chan-Palay, 1973c; Palkovits et al., 1977). Intracerebellar afferents inputs originate from inhibitory PC projections, local interneurons, and from excitatory recurrent collaterals from other nuclear cells (Eager, 1968; O'Leary et al., 1972; Chan-Palay, 1973d).

Unlike the cerebellar cortex, our understanding of the connectivity and neuronal types in the DCN is still incomplete. Most recently, a large glycinergic neuron that projects to the brain stem has been identified in the FN (Bagnall et al., 2009). In addition, two types of glycinergic neurons were identified in the DCN, a spontaneously active interneuron and an inactive neuron (at rest) that projects to the cerebellar cortex (Uusisaari and Knopfel, 2010). Despite the large amount of research focused on the cerebellum, our knowledge about the internal neuronal and synaptic organization of the DCN remains unclear.

\section{Learning mechanisms in the cerebellum}

The repetitive and homogeneous organization of the different neurons of the cerebellar cortex was described in detail by Ramon and Cajal (Ramón y Cajal, 1888, 1889). However, the role and function remained only speculative for over five decades because of the lack of knowledge about the properties of individual neurons. A new era in cerebellar circuitry was initiated by the work of the Nobel price laureate, Sir John Eccles in the early 1960s. After his work on the spinal cord, he focused his innovative recording techniques on the characterization of the properties of different neurons in the cerebellar cortex. About that time and after having studied with Eccles, Masao Ito made the discovery that Purkinje cells are in fact inhibitory (Ito et al., 1964). With the contribution from the Szentágothai laboratory characterizing the anatomical and morphological 
properties of individual cerebellar neurons, in 1967 Eccles, Ito, and Szentágothai published "The Cerebellum as a Neuronal Machine" (Eccles, 1967).

Despite a comprehensive knowledge of its components, the mechanism by which the cerebellum processes information to generate a precise, coordinated output remains unknown. To date, it is not clear how information from PCs is integrated on their target nuclear cells to generate movements. Purkinje cells fire at $\sim 50-100 \mathrm{~Hz}$ and form numerous inhibitory synapses onto their target nuclear cells. Despite the large amount of inhibitory input from tonically active PCs, most nuclear cells fire at $\sim 40 \mathrm{~Hz}$ (Aizenman and Linden, 1999; Raman et al., 2000; Anchisi et al., 2001; Uusisaari et al., 2007).

The simplicity and stereotyped architecture of the cerebellar cortex motivated the development of theoretical models of cerebellar function. The Marr-Albus theory postulates that PCs adjust and correct movements by detecting the temporal pattern and sequence of PF and CF signals (Marr, 1969; Albus, 1971). In the early 1980s Masao Ito described for the first time long term depression (LTD) in the cerebellar cortex. Coincidence of PF and CF inputs on Purkinje cells leads to LTD on the PF synapses (Ito et al., 1982). It was postulated that LTD on PF synapses would result in a decrease of Purkinje cell activation and consequently on disinhibition of its target nuclear cells. It has been hypothesized that a pause in inhibitory, tonically active Purkinje cells allows their target nuclear cells to produce the necessary firing activation required to generate a motor command. For over two decades, depression of PF synapses onto PCs and subsequent disinhibition of their target nuclear neurons was the prevailing paradigm of cerebellar learning.

There is an ongoing controversy about the location of the cerebellar cortex involved in EBC. Some earlier studies in the rabbit using permanent lesions, infusions of AMPA receptor antagonists or single and multi unit recordings identified an area in cerebellar cortex lobule HVI involved in EBC (Yeo et al., 1985a; Berthier and Moore, 1986; Gould and Steinmetz, 1996; Attwell et al., 2001). In addition, other areas were identified in lobule V, VII, the anterior, and paramedian lobule (Garcia et al., 1999; Green and Steinmetz, 2005; Kalmbach et al., 2010). In vivo recording studies in the cerebellar cortex found groups of Purkinje cells that change their firing pattern in response to a tone-CS, or an airpuff-US, and some responded to the presentation 
of both stimuli. However, the firing rate of Purkinje cells did not seem to correlate with the formation and execution of CRs but it seemed to rather encode different features of the CRs (Berthier and Moore, 1986; Hesslow, 1994a; Jirenhed et al., 2007). Recording and stimulating studies from different locations in the cerebellar cortex identified several eyeblink microzones that receive $\mathrm{CF}$ input from corneal stimulation and when stimulated can generate eyeblink responses (Yeo et al., 1985a; Hesslow, 1994b; Green and Steinmetz, 2005; Jirenhed et al., 2007; Mostofi et al., 2010). While Purkinje cell firing profiles seem to encode different properties of the $\mathrm{CR}$, the firing profile of AIN neurons precisely reflects the motor output. It would appear that Purkinje cells in a functional module or microcomplex would encode information from a particular body part and multiple sensory modalities, while their target nuclear cells are in charge of integrating this information to control the appropriate musculature. Because AIN neurons are the final output from the cerebellum and they play a critical role in $\mathrm{EBC}$, they are the focus of the current research.

More recent studies in transgenic mice revealed that blocking LTD at the PF-PC synapse does not impair movement or learning of different cerebellar-dependet tasks including EBC and vestibular ocular reflex (Schonewille et al., 2011), suggesting that the cerebellum relies on other mechanisms to encode learning. Intracellular recordings described a peculiar response in nuclear cells as a result of synchronized bursts of Purkinje cell inputs. However, when Purkinje cells fire in trains of action potentials or bursts, it produces a strong inhibitory effect (hyperpolarization) that sometimes is followed by a rebound burst of action potentials depolarization. During a rebound depolarization a nuclear cell increases its firing rate and can fire bursts of action potentials. It has been proposed that this is the mechanism by which the cerebellum generates motor commands.

The AIN is somatotopically organized. The lateral portion of the AIN controls musculature of the face, neck, and upper body while the medial portion of the AIN controls musculature of the lower body (Ekerot and Larson, 1979; Mojtahedian et al., 2007). In EBC, a small region in the dorsolateral AIN is necessary for the formation and execution of CRs. A lesion as small as a cubic millimeter in this region is sufficient to abolish the formation of CRs while the performance of the UR is not affected (Lavond et al., 1984). In vivo stimulation of the 
dorsolateral AIN before EBC can generate eyeblink responses, suggesting a pre-existing functional connection to eyeblink premotor pathways (McCormick and Thompson, 1984a; Berthier and Moore, 1990; Freeman and Nicholson, 2000). Lesion and inactivation studies show that the AIN is critical for the formation of CRs (Lavond et al., 1984; Steinmetz et al., 1992; Krupa and Thompson, 1997). However, direct evidence comes from single unit recording studies in the AIN during EBC. Recordings show that some neurons change their pattern of activation during paired stimulus presentations that correlates with the development of CRs (McCormick and Thompson, 1984a; Berthier and Moore, 1990; Tracy et al., 2001). These neurons increase their firing rate during the execution of CRs in a pattern that precedes and predicts the performance of eyeblink CRs (McCormick and Thompson, 1984a). Moreover, blocking protein synthesis prevents the formation of CRs (Bracha et al., 1998). In sum, the AIN is involved and necessary for the formation and execution of CRs.

There is no argument about the importance of AIN neurons in the formation and execution of CRs; however, direct evidence of learning-specific changes on these neurons has been elusive. Learning at a neuronal level can be accomplished by a number of mechanisms including synaptic plasticity and changes in membrane excitability. Synaptic plasticity refers to synaptic changes that can range from electrophysiological properties of synapses to morphological changes on neuronal connectivity such as synaptogenesis and pruning. Intrinsic membrane excitability refers to changes in neuronal membrane conductance as a result of the expression of voltagedependent channels (Schreurs et al., 1991; Schreurs et al., 1998). It has been suggested that an increase in membrane excitability may set the neuron to a permissive state to facilitate synapse remodeling (Mozzachiodi and Byrne, 2010).

During EBC, repeated pairings of a tone-CS and an airpuff-US lead to an association between these two stimuli and consequently, the tone-CS becomes capable of eliciting an eyeblink. Information about the tone is conveyed to the AIN by MF collaterals which make excitatory synapses onto nuclear cells. A possible mechanism underlying the formation of CRs in the AIN involves an increase in the MF input. It has been proposed that MFs that convey information about the tone-CS will undergo synaptic and structural changes during EBC including potentiation and synapse formation (Kleim et al., 2002; Pugh and Raman, 2006; Weeks et al., 
2007; Boele et al., 2013). An increase in the MF input will allow the tone-CS to activate nuclear cells and generate a learned eyeblink response.

Current electrophysiological studies in this region have provided a better understanding of the properties of nuclear cells and some possible mechanisms for plasticity that can be translated into motor learning (Pugh and Raman, 2006; Person and Raman, 2010). However, direct evidence of MF plasticity in the AIN has been hard to find until recently. A groundbreaking electrophysiology study showed for the first time that MF synapses can undergo long-term potentiation (LTP) during an induction protocol that resembled the sequence of excitatory and inhibitory inputs expected to occur during EBC (Pugh and Raman, 2006). In this protocol, stimulation of MFs followed by stimulation of Purkinje cells induced LTP on the MF synapses of nuclear cells. Interestingly, this form of LTP appears to be activity-dependent and synapsespecific because only synapses that were activated before Purkinje cell stimulation underwent potentiation (Pugh and Raman, 2008; Pugh and Raman, 2009). Whether or not LTP at the MF synapse accurately reflects the neuronal processes involved in EBC remains unknown, but it provides a reasonable mechanism that can lead to an increase in the MF input.

Evidence of a possible increase in MF input comes from two electron-microscope studies that examined excitatory and inhibitory synapses in the interpositus nucleus. Interestingly, excitatory synapses increased in length or in number after EBC (Kleim et al., 2002; Weeks et al., 2007). These data suggest that excitatory synapses in the interpositus nucleus are susceptible to EBC and can undergo structural changes as a function of learning. However, the various neuronal components and the complex interconnectivity found in the interpositus nucleus make the origin and source of these synapses unclear. In addition, because the interpositus nucleus is somatotopically organized, it is probable that most synapses belong to neurons that are not involved in the eyeblink pathway. What would be expected is that EBC-related changes would occur only on neurons that are involved in EBC. 


\section{Pseudorabies virus as a transneuronal tracer}

The fairly recent development of transsynaptic viral tracers has proven a useful tool for the delineation of functional pathways. Pseudorabies virus, PRV, is a swine herpesvirus of the Alphaherpesvirinae subfamily, also known by its taxonomic name, suid herpesvirus 1 , or by its original name, Aujeszky's disease virus. PRV is not neuroinvasive in its natural swine host, but it is neurotropic in a wide range of vertebrates and it has been used to study neuronal pathways in rats, mice, rabbits, hamsters, dogs, cats, ferrets and other non-primate species (Fay and Norgren, 1997; Chien et al., 1998; de Groat et al., 1998; Pickard et al., 2002; Billig et al., 2003; Brittle et al., 2004). Despite its significant homology to human alpha-herpes viruses and its broad host range, PRV is not transmitted to humans (Pomeranz et al., 2005). Even though PRV does not infect humans, experimental studies in nonhuman primates indicate that marmosets and rhesus monkeys can be susceptible to infection; however, other higher-order primates such as chimpanzees are not (Enquist, 1999).

Figure 7 shows the advantage of retrograde viral transsynaptic tracers over traditional tracers for the delineation of circuits. The most efficient PRV neuronal tracers are derived from attenuated PRV strains with reduced virulence because wild-type PRV is extremely virulent. PRV-Bartha is derived from a live PRV vaccine and is one of the best characterized attenuated PRV strains (Bartha, 1961). Another characteristic of PRV-Bartha is that it spreads only retrogradely and allows for identification of efferent pathways. In addition, PRV does not label sensory pathways, and even though it can be taken up by sensory neurons, its exclusively retrograde properties confine the virus to the sensory neuron soma where it remains without spreading any further (Smith et al., 2000; Mettenleiter, 2002; Mettenleiter et al., 2006). Astrocytes are susceptible to PRV infection but are not permissive for viral replication and do not contribute to trans-neuronal spread of the virus (Card, 1998). 


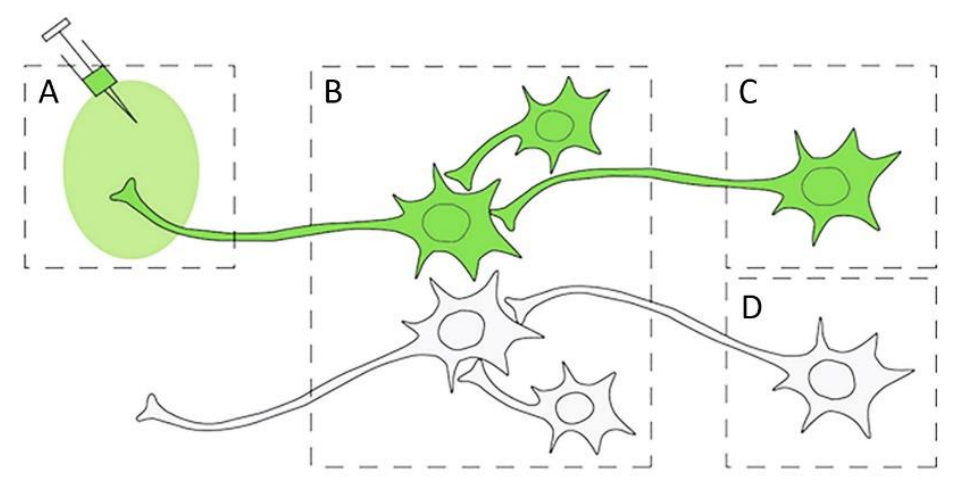

B Tracing using non-transsynaptic conventional tracer

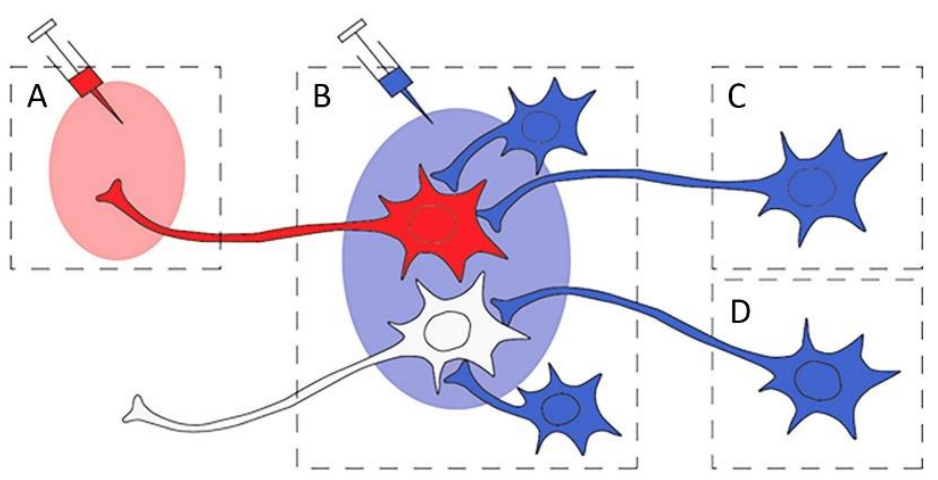

Figure 7. Pseudorabies virus as a retrograde transneuronal tracer.

Schematic diagram of neuronal pathways revealed by retrograde transneuronal viral tracers or conventional tracers. (A) By injecting a retrograde viral tracer into Region A, the virus will first infect the neurons that directly project to the injection site (first-order neuron) in Region B, and then spread further to the synaptically connected upstream neurons (secondorder neuron) in Region C. Therefore, transsynaptic labeling enables us to trace chains of specific neurons. (B) By using conventional non-transsynaptic tracers, several experiments with different injection sites must be conducted in order to reveal circuits: injection to Region A to detect first-order neurons, and injection to Region B to detect second-order neurons. However, neurons that are not connected with the circuit A may also be stained, such as the neuron in Region D, adapted from (Ohara et al., 2009).

PRV-Bartha is a superior transneuronal tracer and has been shown to faithfully reproduce the results obtained using other well-characterized monosynaptic nonviral tracers. PRV enters the neuron terminals by fusion. Once inside, the virus caspids interact with the neuron's microtubule-associated motor protein, dynein, and travels retrogradely to the nucleus where it 
replicates. After replication, PRV moves to the dendritic tree where it infects synaptically connected neurons. More importantly, PRV does not spread to synaptically unconnected but physically adjacent processes, exclusively mapping functional connected pathways. PRV-Bartha is a powerful tool for the delineation of neuronal circuits. Anatomical and synaptically connected networks can be easily identified using the temporal and hierarchal spread of PRV. In addition, PRV has been extensively used to trace various efferent pathways and appears to be suitable for delineating the eyeblink premotor pathway (Travers and Rinaman, 2002; Jovanovic et al., 2010; Johnson et al., 2011; Van Daele et al., 2011; Rubelowski et al., 2013). 


\section{Chapter 3. Rabbit eyeblink premotor pathway}

\section{Introduction}

Delay EBC is a well-characterized model of associative learning but the locus of learning is still a matter of debate. Possible sites for plasticity have been found in the AIN of the DCN (McCormick et al., 1982b; Krupa et al., 1993; Thompson, 2013), lobule HVI of the cerebellar cortex (Yeo et al., 1985b; Gould and Steinmetz, 1996; Schreurs et al., 1998; Jirenhed et al., 2007; Kellett et al., 2010), and anterior lobule V (Perrett and Mauk, 1995; Green and Steinmetz, 2005). Identification of a complete cerebellar premotor pathway of the eyeblink response can be of great value in elucidating the specific components involved in the generation and modulation of EBC.

DCN is the final filter that integrates all the information processed in the cerebellum and generates its sole output. However, unlike the cerebellar cortex, the circuitry of the DCN is poorly understood. There are at least six types of neurons in the DCN including large glutamatergic projection neurons, two types of inhibitory projection neurons, and at least two types of interneurons. The only excitatory output of the DCN is generated by large glutamatergic neurons that project to different extra-cerebellar sites and comprise about $50 \%$ of the total DCN neuronal population (Batini et al., 1992; Teune et al., 2000). The first type of inhibitory projection neuron is a small GABAergic neuron that projects to the inferior olive and forms the nucleo-olivary feedback loop (Tolbert et al., 1976; De Zeeuw et al., 1989). The second type is a large glycinergic neuron (recently discovered in the fastigial nucleus) that projects to vestibular and reticular sites (Bagnall et al., 2009). Recent electrophysiology studies in transgenic mice expressing GAD-eGFP or GlyT2-eGFP were able to distinguish at least two types of interneurons: a GABAergic or mixed GABA/glycinergic interneuron and a nonGABAergic (possibly glutamatergic) interneuron (Uusisaari and Knopfel, 2010, 2011). However, the role of these interneurons is unknown and their interactions have not been studied. 
Retrograde transsynaptic viral tracers are a powerful tool for identifying neuronal networks. Attenuated pseudorabies virus Bartha, PRV (Bartha, 1961) reveals hierarchical chains of functionally connected neurons and multisynaptic pathways can be identified by following the extent and time course of PRV infection (Daniels et al., 1999; Billig et al., 2000; Van Daele and Cassell, 2009). In the present study, the premotor pathway of the rabbit OOM responsible for the eyeblink was traced retrogradely using PRV. Neurons in the AIN involved in the pathway were characterized based on their neurotransmitter content. Of particular importance, three different types of inhibitory interneurons that are part of the $O O M$ premotor pathway were revealed to form a possible functional network within the AIN for the modulation of motor commands.

\section{Material and Methods}

\section{Animals}

Twenty four adult male New Zealand rabbits (Oryctolagus cuniculus), between 3-4 months of age, weighing approximately $2.5 \mathrm{~kg}$ were supplied by Harlan. Four rabbits were assigned to each of four different time points for PRV anatomical tracing ( 3 days, 4 days, 41/2 days, and 5 days) and four rabbits were assigned to each of two time points for neurotransmitter immunohistochemistry (41/2 days and 5 days). Rabbits were housed in individual cages, given free access to food and water, and maintained on a 12-hr light-dark cycle, all in accordance with the National Institute of Health guidelines. All procedures were approved by the West Virginia University Animal Care and Use Committee.

\section{PRV Injections}

An attenuated PRV expressing an enhanced green fluorescent protein (PRV-152) was used as a retrograde transsynaptic tracer (Smith et al., 2000). The PRV was a kind gift of L. W. Enquist. PRV enters the neuron terminals by fusion. Once inside, the virus caspids interact with the neuron's dynein, a cellular microtubule-associated motor protein, and it travels retrogradely to the nucleus where it replicates. After replication, it moves to the dendrites where it infects synaptically connected cells (Curanovic and Enquist, 2009; Curanovic et al., 2009). PRV does not label sensory pathways, and even though it can be taken up by sensory neurons, its 
exclusively retrograde properties confine the virus to the sensory neuron soma and it does not spread any further (Smith et al., 2000; Mettenleiter, 2002; Mettenleiter et al., 2006). The same batch of PRV was used for all injections at a concentration of $2.3 \times 10^{8}$ plaque-forming units per milliliter (PFU/ml). After arrival, PRV was aliquoted and stored at $-80^{\circ} \mathrm{C}$. Prior to PRV injections, rabbits were anesthetized with $20 \mathrm{mg} / \mathrm{kg}$ Ketamine and $4 \mathrm{mg} / \mathrm{kg}$ Xylazine. Their right eyelid was shaved and cleaned with an isopropol alcohol wipe. After thawing, PRV was sonicated for 2 minutes. A glass, 10- $\mu 1$ Hamilton syringe with a 31-gauge needle was rinsed three times in $95 \%$ ethanol, sterile $\mathrm{dH}_{2} \mathrm{O}$, sterile saline, and PRV. Fifteen microliters of PRV divided into 5 injections of $3 \mu \mathrm{l}$ each were injected into the right $O O M$. Each injection was transverse to the muscle fibers and delivered at a constant rate through the orbital, preseptal, and pretarsal portions of the $O O M$ to ensure adequate viral distribution. After injection, the syringe was left in place for 10 minutes to achieve complete viral absorption and to prevent any leakage.

\section{Tissue Processing}

The sixteen rabbits used for the delineation of the $O O M$ premotor pathway were euthanized with $1.0 \mathrm{cc}$ Euthasol (sodium pentobarbital, $390 \mathrm{mg} / \mathrm{ml})$ at $3(\mathrm{n}=4), 4(\mathrm{n}=4), 4 \frac{1}{2}(\mathrm{n}=4)$ or $5(\mathrm{n}=4)$ days after PRV-injections. They were perfused transcardially with $1.5 \mathrm{~L}$ of $0.9 \%$ saline $(\mathrm{pH} 7.4$ at room temperature) followed by $1 \mathrm{~L}$ of $4 \%$ formaldehyde. For anti-PRV immunohistochemistry, brains were collected and placed in fixative for $4 \mathrm{hrs}$ and transferred to $30 \%$ sucrose for cryoprotection until they sank and $50-\mu \mathrm{m}$ sections were cut on a freezing microtome.

Eight rabbits were used for characterization of AIN neurons based on their neurotransmitter immunoreactivity. Animals were euthanized with $1.0 \mathrm{cc}$ Euthasol (sodium pentobarbital, 390 $\mathrm{mg} / \mathrm{ml})$ at $4 \frac{1}{2}(\mathrm{n}=4)$ or $5(\mathrm{n}=4)$ days after PRV-injections. They were perfused transcardially with $1.5 \mathrm{~L}$ of $0.9 \%$ saline ( $\mathrm{pH} 7.4$ at room temperature) followed by $1 \mathrm{~L}$ of $2.5 \%$ glutaraldehyde and $1.5 \%$ formaldehyde. Brains were placed in fixative for $6 \mathrm{hrs}$, transferred to $30 \%$ sucrose for cryoprotection until they sank and $25-\mu \mathrm{m}$ sections were cut. All sections were marked on the contralateral side of injection to distinguish laterality.

\section{Anti-PRV Immunohistochemistry}

Free-floating sections were washed in $0.5 \mathrm{M}$ Tris and placed in $3 \% \mathrm{H}_{2} \mathrm{O}_{2}$ for 30 minutes to quench endogenous peroxidases. Antigen retrieval was accomplished by incubating the sections 
in citrate buffer ( $\mathrm{pH} 3.0)$ for 30 minutes at $37^{\circ} \mathrm{C}$. After washing, the sections were blocked for $1 \mathrm{hr}$ in $3 \%$ normal rabbit serum and incubated in a goat anti-PRV primary antibody overnight at $4^{\circ} \mathrm{C}$ (polyclonal gb-320 developed by R. Miselis, 1:10,000). The PRV primary antibody has been described extensively (Chen et al., 1999; Aston-Jones and Card, 2000; Aston-Jones et al., 2004; James et al., 2008). After washing, the sections were placed in a secondary antibody (biotinylated rabbit anti-goat 1:200) for $1 \mathrm{hr}$. The sections were then washed and placed in ABC (Vector Kit, Vector Labs) for $1 \mathrm{hr}$, washed once more, and stained with DAB (Vector Kit, Vector Labs) for 2-4 min until color developed.

\section{Fluorescence Neurotransmitter Immunoreactivity}

The $25-\mu \mathrm{m}$ free floating sections were treated with $0.5 \%$ sodium borohydride in $0.1 \mathrm{M} \mathrm{PBS}(\mathrm{pH}$ 7.2) for 30 minutes to quench autofluorescence produced by glutaraldehyde and followed by repeated washes. Sections containing the DCN were incubated with primary antibodies for 72 hrs and with secondary antibodies for $48 \mathrm{hrs}$ at $4^{\circ} \mathrm{C}$ in a buffer solution containing $0.1 \mathrm{M}$ PBS ( $\mathrm{pH} 7.2$ ), $3 \%$ normal goat serum and $0.1 \%$ sodium azide (preservative). After specificity tests, an optimal immunolabeling protocol was developed. First, sections were incubated in a polyoclonal goat anti-PRV (gb-320 1:10,000; R. Miselis) followed by its secondary antibody (donkey anti-goat Alexa 488, Invitrogen; 1:500). Second, sections were incubated in a mixture of monoclonal mouse anti-L-glutamate conjugated to glutaraldehyde and bovine serum albumin (anti-Glu-G-BSA) (Millipore MAB5304, 1:1,000), polyclonal guinea pig anti-GABA conjugated to glutaraldehyde and keyhole limpet hemocyanin (anti-GABA-G-KLH) (Millipore AB175, 1:1,000) and polyclonal rabbit anti-glycine conjugated to glutaraldehyde and BSA (anti-Gly-GBSA) (Millipore AB139, 1:1,000). After primary antisera incubation, sections were washed several times over $24 \mathrm{hrs}$ with $0.1 \mathrm{M}$ PBS and $0.1 \%$ sodium azide. Sections were then incubated with secondary antibodies (goat anti-rabbit Alexa 405, goat anti-mouse Alexa 546, and goat antiguinea pig Alexa 633; Invitrogen, 1:400). The sections were then washed and mounted on gelatin-covered mounting slides and cover-slipped using Fluoromount-G mounting media (SouthernBiotech) and \#1.5 cover slips (Fisher Scientific). 


\section{Antibody characterization}

Monoclonal mouse anti-Glutamate antiserum (Millipore, MAB5304) has been previously characterized in several studies (Chagnaud et al., 1989; Roche et al., 2003; Wu et al., 2005). Specificity testing by competition experiments using ELISA carried out by the manufacturer showed no cross-reactivity with aspartate-G-BSA, GABA-G-BSA or Gly-G-BSA. Polyclonal guinea pig anti-GABA antiserum (Millipore, AB175) has been used to identify GABAergic neurons by light and electron microscopy (manufacturer's technical information) and tested in dot blots showing specificity for its conjugated antigen and immunoreactivity can be removed by preabsorption with its antigen (Chalazonitis et al., 2008). In addition, anti-GABA-G-KLH has been extensively characterized in different preparations and species (McDonald and Pearson, 1989; Roettger et al., 1989; Dmitrieva et al., 2001; Rubio and Juiz, 2004; Stanic et al., 2010). The polyclonal rabbit anti-Glycine antiserum (Millipore, AB139) has been used in different tissue preparations and species (Avendano et al., 2005; Fredrich et al., 2009; Kuo et al., 2009; Downie et al., 2010). Specificity testing by competition experiments using ELISA carried out by the manufacturer showed no cross-reactivity with GABA-G-BSA, Glu-G-BSA, taurine-G-BSA or aspartate-G-BSA. The specificity of this antiserum was also tested by western blotting in sea lamprey brain homogenates and showed high specificity for Gly-G-BSA (Villar-Cervino et al., 2006).

\section{Controls and specificity tests}

In the present study additional neurotransmitter antisera specificity tests were performed. First, omission controls in which the primary antibodies were replaced with incubating buffer eliminated immunoreactivity. Second, preabsorption of the primary antibodies with the immunizing peptide (purchased from Invitrogen or Vector Labs) significantly decreased the amount of immunoreactivity. Third, preabsorption of each primary antibody with the other two conjugated peptides did not reduce immunoreactivity (e.g. $5 \mu 1$ of anti-GABA-G-BSA preabsorbed with $1 \mu 1$ of glycine-G-BSA and $1 \mu 1$ of glutamate-G-BSA did not reduce immunoreactivity for GABA, the same was true for the other two conjugated antibodies). In addition, identical immunoreactivity was seen in experiments where the three preabsorbed primary antibodies were used sequentially compared to experiments where primary antibodies were used simultaneously. All control tests were carried out on the cerebellar cortex where the 
neurotransmitter profile of cells has been well-characterized and where granule cells are exclusively glutamatergic, stellate and basket cells are GABAergic, globular cells are glycinergic and Golgi cells are both GABAergic and glycinergic (Ottersen et al., 1988; Simat et al., 2007).

\section{Image acquisition}

Light microscopy. Images were acquired using an upright Olympus AX70 light microscope with a motorized stage and with $4 X$ (NA 0.15) or 10X (NA 0.4) objectives. Some pictures of different brain structures were acquired using the virtual slice module to produce high resolution images (WVU, Microscopic Imaging Facility).

Confocal microscopy. Neurotransmitter immunofluorescent reactivity of the DCN of rabbits previously injected with PRV (41/2 and 5 days) was visualized using a confocal laser scanning microscope (Zeiss LSM 710; Carl Zeiss International). Images were acquired using 405, 488, 514 , and 633nm lasers, sequential multichannel line scan, averaged twice, pinhole aperture of 1AU, 1024x1024 resolution and the filters were set manually to detect the spectral peak of each fluorophore. All coronal sections $(25 \mu \mathrm{m})$ of the DCN were visualized first using a 10X objective (NA 0.4) to locate all PRV-labeled neurons. Only PRV-labeled neurons with a visible nucleus were included in this study to standardize data acquisition and to avoid over counting. Classification of DCN cell neurotransmitter immunoreactivity was performed using a 63X oil immersion objective (NA 1.4). Images were exported to Adobe Photoshop 8.0 and only minor adjustments of brightness and contrast were made.

\section{Results}

The directional and temporal pattern of PRV retrograde spread through synaptically connected circuits can be used to identify specific pathways. In the present study, four different time points were used to identify each step in the rabbit $O O M$ premotor pathway. Based on pilot data, 4 time points were selected to best represent the progression of PRV labeling through each step in the pathway ( 3 days $n=4,4$ days $n=4,41 \frac{1}{2}$ days $n=4$ and 5 days $n=4$ ). A basic diagram of the PRV procedures is depicted in Figure 8. 


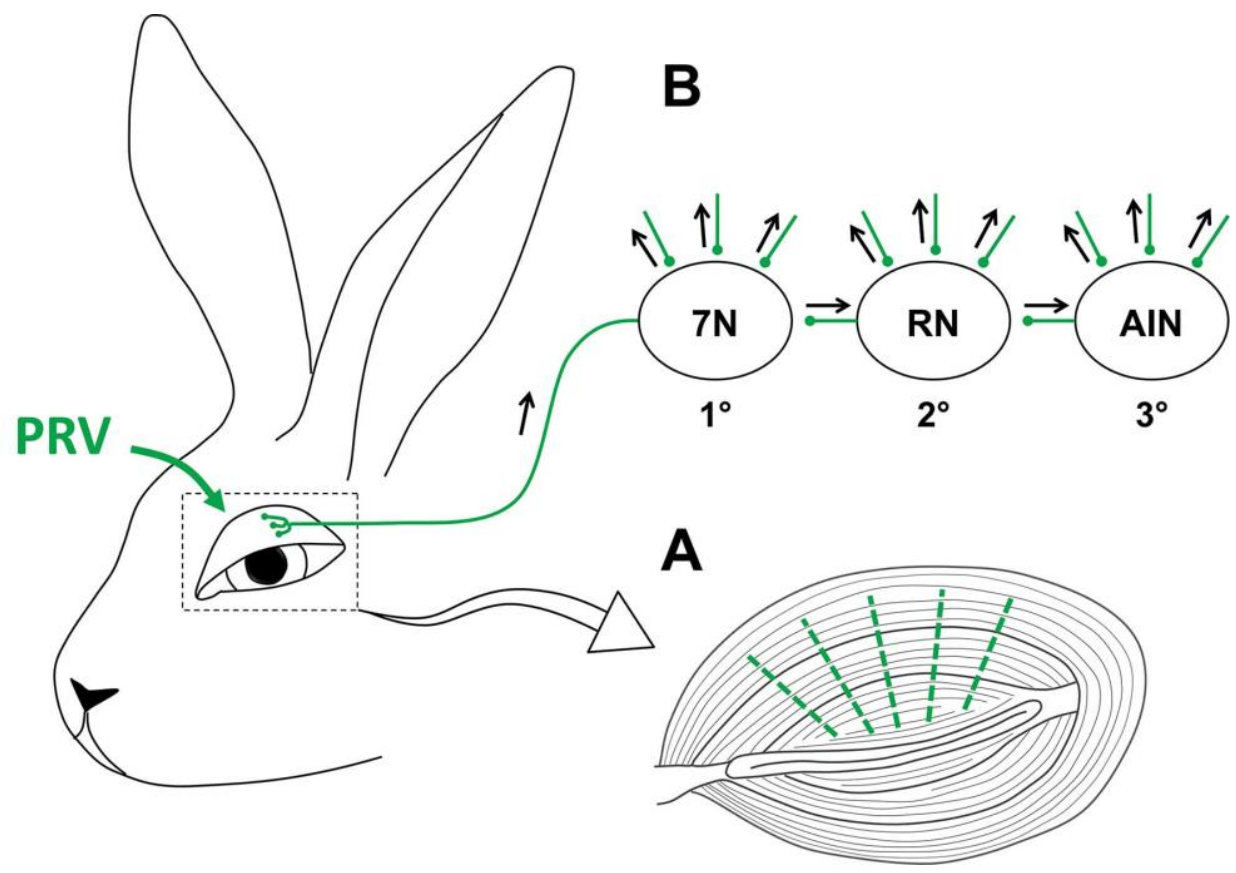

Figure 8. Schematic diagram of the eyeblink premotor pathway labeled with PRV. A shows the location of 5 PRV injections (green dashed lines) in OOM that include the orbital, preseptal, and pretarsal muscles. B shows the retrograde transsynaptic spread of PRV through the OOM premotor pathway. Directional and sequential spread of PRV through first-order $\left(1^{\circ}\right)$ motor neurons in the facial nucleus $(7 \mathrm{~N})$, second-order $\left(2^{\circ}\right)$ premotor neurons in the red nucleus $(\mathrm{RN})$ and other areas, and third-order $\left(3^{\circ}\right)$ premotor neurons in the AIN of the cerebellum and other areas.

\section{Mapping the OOM premotor pathway}

Three days after PRV injections in the OOM, first-order motoneurons labeled with PRV were seen in the ipsilateral facial nucleus (Figure 9 A). Labeled neurons were located in the dorsolateral and dorsal portions of the intermediate and lateral subdivisions of the nucleus forming a cap-like distribution in agreement with the location of motoneurons innervating the OOM found in previous studies (Radpour, 1977; Komiyama et al., 1984; Klein et al., 1990; VanderWerf et al., 1998; Horta-Junior et al., 2004; McNeal et al., 2008).

Importantly, the same region of the nucleus was labeled in all rabbits. In addition, neuronal degeneration was evident in this region at $4 \frac{1}{2}$ and 5 days after PRV injection. Exclusive PRV 
up-take by $O O M$ motoneurons was corroborated by examination of motor and preautonomic structures known to innervate other ocular muscles and glands. The abducens nucleus that innervates extraocular muscles was not labeled with PRV at any of the time points examined (Figure 9 B). In addition, the superior lacrimal and salivatory nucleus, which contains preganglionic autonomic efferents to the eyelid glands and smooth muscle, was also not labeled with PRV in any of the subjects used in this study at any of the time points (Figure 9 B). The facial nucleus and other structures labeled with PRV at 3, 4, 41/2, and 5 days after PRV injections are shown in Table 1.

Table 1. Labeled areas after PRV injection into the right orbicularis oculi muscle of the eyelid.

\begin{tabular}{|c|c|c|c|}
\hline \multicolumn{4}{|c|}{ Days after PRV injection } \\
\hline 3 days $(n=4)$ & 4 days $(n=4)$ & $4 \frac{1}{2}$ days $(n=4)$ & 5 days $(n=4)$ \\
\hline \multirow[t]{12}{*}{ Facial Nucleus ${ }^{(\mathrm{ppsi})}$} & All areas previously labeled + & All areas previously labeled + & All areas previously labeled + \\
\hline & Medullary reticular nuclei ${ }^{\left(\mathrm{Ipsi} \mathbf{i}^{+}+\mathrm{Cont}\right)}$ & Anterior Interpositus nucleus ${ }^{(\mathrm{ppsi})}$ & Fastigial nucleus ${ }^{(\mathrm{ppsi}+\mathrm{Cont})}$ \\
\hline & Spinal trigeminal nucleus ${ }^{\left(\mathrm{Ipsi} \mathrm{s}^{*}+\text { Cont }\right)}$ & Fastigial nucleus ${ }^{\text {(Ipsi) }}$ & Interpositus nucleus ${ }^{\left(\mathrm{Ipsi} \mathrm{s}^{*+} \text { Cont) }\right.}$ \\
\hline & Nucleus of the solitary tract ${ }^{(\mathrm{lpsi})}$ & Trigeminal motor nucleus ${ }^{(\mathrm{ppsi})}$ & Dentate nucleus ${ }^{\left(\mathrm{Ipsi} \mathbf{s i}^{*+} \text { Cont }\right)}$ \\
\hline & Periolivary nuclei ${ }^{(\mathrm{Ipsi})}$ & Lateral vestibular nucleus ${ }^{\left(\mathrm{lpsi} \mathbf{p}^{*}+\mathrm{Cont}\right)}$ & Cerebellar cortex ${ }^{(\mathrm{ppsi})}$ : lobule I and VI \\
\hline & Trapezoid body ${ }^{(\mathrm{ppsi})}$ & Lateral lemniscus ${ }^{(1 p s i *+C o n t)}$ & Cunate nucleus ${ }^{(\mathrm{ppsi}+\text { Cont })}$ \\
\hline & Cochlear nucleus ${ }^{(\mathrm{Ipsi})}$ & Oculomotor nucleus ${ }^{(\mathrm{ppsi}+\text { Cont) }}$ & Gracile nucleus ${ }^{(1 p s i *+C o n t)}$ \\
\hline & Kölliker-fuse nucleus ${ }^{(\mathrm{Ipsi})}$ & Periaqueductal gray ${ }^{(\mathrm{Ipsi}+\mathrm{Cont})}$ & Deep mesencephalic nucleus ${ }^{(\mathrm{Ipsi}+\mathrm{Cont})}$ \\
\hline & Parabrachial nuclei ${ }^{(\mathrm{lpsi})}$ & Interstitial nucleus of Cajal ${ }^{\text {(Ipsi+Cont) }}$ & Retrorubral field ${ }^{\text {(Ipsi+Cont) }}$ \\
\hline & Medial vestibular nucleus ${ }^{(\mathrm{Ipsi})}$ & Nucleus of Darkschewitsch ${ }^{(\text {Ipsi+Cont })}$ & Superior colliculus ${ }^{\text {(Cont) }}$ \\
\hline & Red nucleus ${ }^{(\text {Cont) }}$ : dorsolateral & & Substantia nigra ${ }^{\left(\mathrm{Ipsi}{ }^{*}+\mathrm{Cont}\right)}$ \\
\hline & Pararubral nucleus ${ }^{\text {(Cont) }}$ & & \\
\hline
\end{tabular}

Four days after PRV injection, premotor structures were labeled. A group composite of PRVlabeled neurons was plotted in a map of coronal sections (Figure 10) to best represent the distribution of labeled neurons at this time. PRV-labeled neurons were found in the dorsal, intermediate, ad ventral medullary reticular nucleus predominately ipsilaterally but some were seen on the contralateral side. 


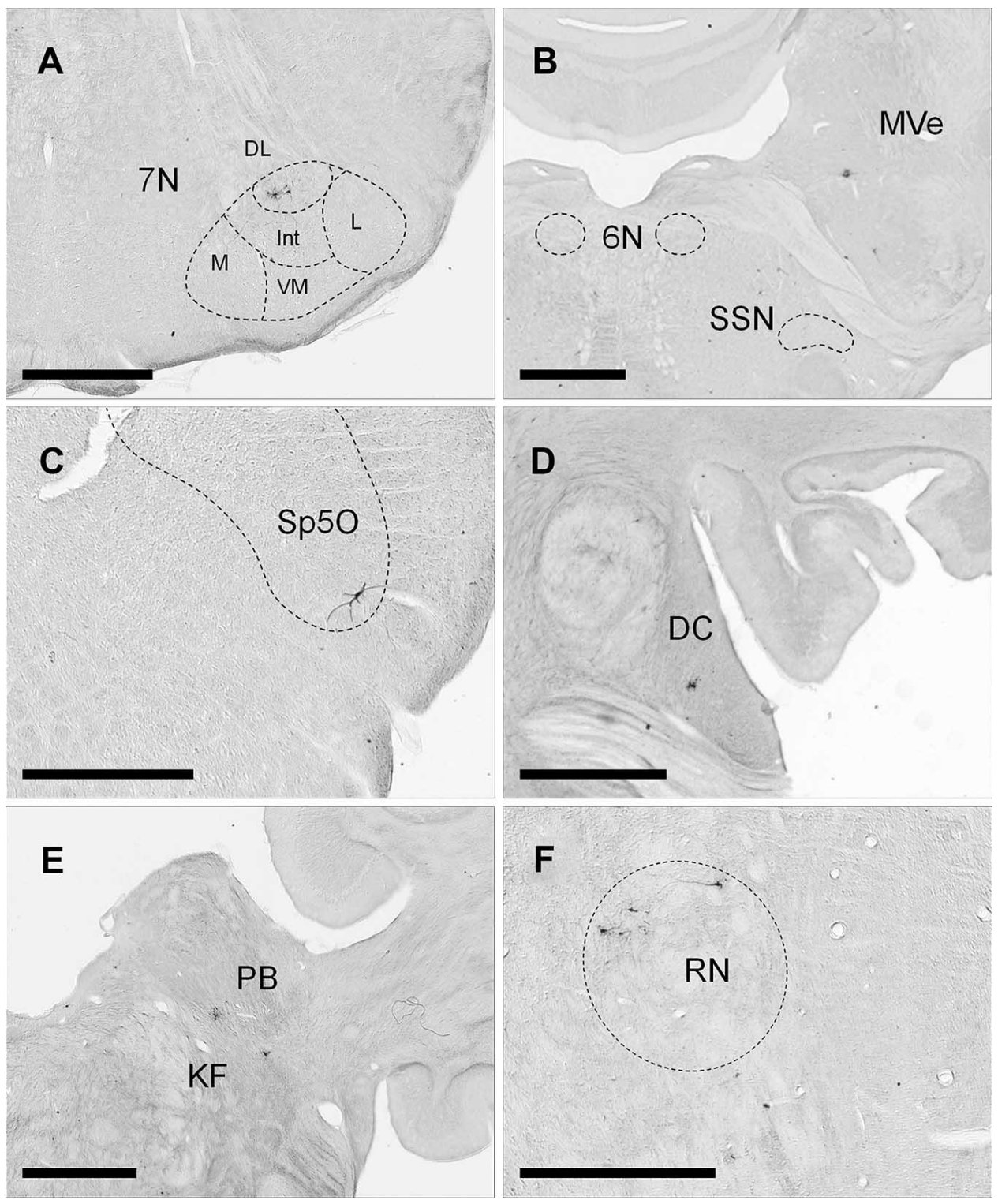

Figure 9. Examples of PRV-labeled neurons 3 and 4 days after PRV injection into the OOM.

A, PRV-labeled first-order motor neurons are located in the dorsolateral facial nucleus ipsilaterally $3 \mathrm{~d}$ after PRV injection into the right OOM. DL, Dorsolateral; L, lateral; Int, intermediate; M, medial; VM, ventromedial subnuclei. B-F, PRV-labeled neurons $4 \mathrm{~d}$ after PRV injection. B shows PRV-labeled neurons in the medial vestibular nucleus (MVe) ipsilaterally and absence of PRV labeling in the superior salivatory nucleus (SSN) and abducens nucleus (6N). C shows PRV labeling in the ventral spinal trigeminal nucleus, oral part (Sp5O) ipsilaterally. D, Discrete PRV labeling in the rostral dorsal cochlear nucleus (DC) ipsilaterally. E shows PRV-labeled neurons in the parabrachial nucleus (PB) and Kolliker-fuse nucleus (KF) ipsilaterally. F shows PRV-labeled neurons in the dorsolateral red nucleus (RN) contralaterally. Scale bars: $1 \mathrm{~mm}$. 
In addition, labeled neurons were found in the magnocellular, gigantocellular, lateral paragigantocellular, parvicellular, and lateral reticular nucleus mostly ipsilaterally. Vestibular second-order labeled neurons at this time were seen only in the ventral part of the medial vestibular nucleus ipsilaterally (Figure 9 B). In addition, labeled neurons were found in the medial and ventral portions of the caudal, interpolar, and oral spinal trigeminal nucleus and in the ventral part of the principal sensory trigeminal nucleus exclusively on the ipsilateral side, in agreement with the location of spinal and principal sensory trigeminal neurons that have been shown to make monosynaptic connections to the facial motor nucleus and generate reflex eyeblink responses (Hinrichsen and Watson, 1983; Yokota et al., 1991; van Ham and Yeo, 1996b, a; Henriquez and Evinger, 2007). Figure 9 C depicts a characteristic neuron located in the ventral spinal trigeminal nucleus (oral part). Second-order auditory areas including the nucleus of the trapezoid body, periolivary nuclei, and dorsal cochlear nucleus were labeled ipsilaterally at this time. The nucleus of the trapezoid body was labeled mostly on the dorsolateral side. A few labeled neurons were seen in the medial and lateral periolivary nuclei. The rostral portion of the dorsal cochlear nucleus was also labeled with PRV on the ipsilateral side at this time (Figure 9 D). A discrete group of labeled neurons were found in the Köllikerfuse nucleus and the ventral part of the medial parabrachial nucleus on the ipsilateral side (Figure 9 E). The Kölliker-fuse and the parabrachial nucleus are involved in the modulation of breathing and it may play a role in synchronizing OOM activity and respiration (Chamberlin and Saper, 1994; Bonis et al., 2010; Song et al., 2012). Finally at this time point, labeled neurons were seen in the dorsolateral RN on the contralateral side (Figure 9 F). These neurons were located in the caudal portion of the magnocellular RN, a region that has been shown to innervate the $O O M$ portion of the facial nucleus and can generate blink responses when stimulated (Takada et al., 1984; Daniel et al., 1987; Chapman et al., 1988; Holstege and Tan, 1988). In addition, a small number labeled neurons were found in the adjacent contralateral pararubral nucleus. 


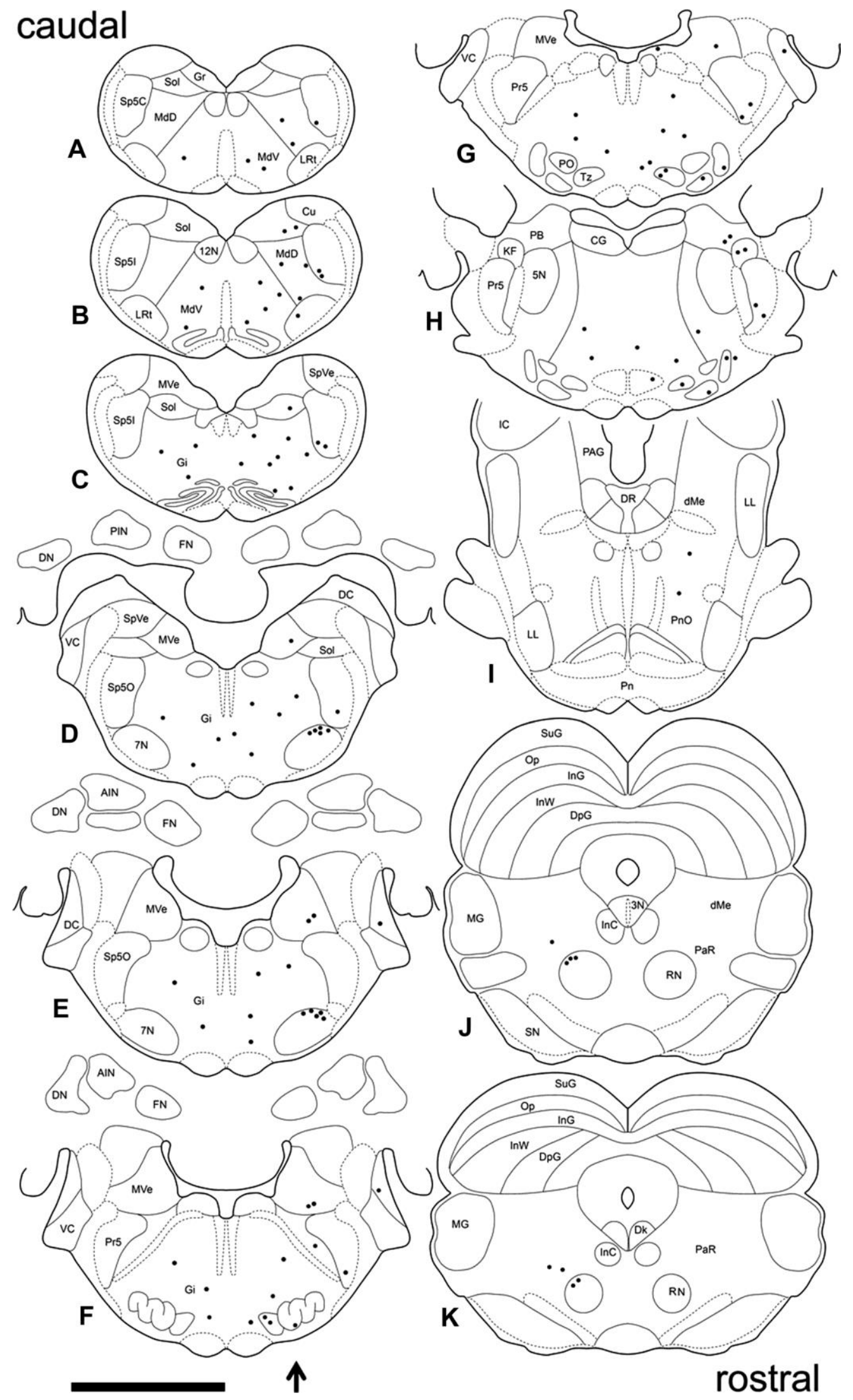


Figure 10. Distribution of PRV-labeled neurons 4 days after PRV injection into the OOM. A composite of the location of PRV-labeled neurons found across four animals plotted in sequential sections to best represent the pattern of PRV labeling. Sections are arranged caudorostrally (A-K ). The arrow indicates the side of PRV injection. Each dot represents one PRV-labeled neuron. 3N, Oculomotor nucleus; 4N, trochlear nucleus; $5 \mathrm{~N}$, motor trigeminal nucleus; 7N, motor facial nucleus; $12 \mathrm{~N}$, hypoglossal nucleus; AIN, anterior interpositus; CG, central gray; Cn, cuneiform nucleus; DC, dorsal cochlear; Dk, nucleus of Darkschewitsch; LL, lateral lemniscus; dMe, deep mesencephalic nucleus; DN, dentate nucleus; PAG, periaqueductal gray; DpG, deep layer of the superior colliculus; FN, fastigial nucleus; Gi, gigantocellular reticular nucleus; Gr, gracile nucleus; IC, inferior colliculus; InC, interstitial nucleus of Cajal; InG, intermediate gray layer of the superior colliculus; InW, intermediate white layer of the superior colliculus; KF, Ko"lliker-fuse nucleus; LRt, lateral reticular nucleus; MdD, dorsal medullary reticular nucleus; MdV, ventral medullary reticular nucleus; MG, medial geniculate nucleus; MVe, medial vestibular nucleus; Op, optic nerve layer of the superior colliculus; PaR, pararubral nucleus; PB, parabrachial nucleus; Pn, pontine nucleus; $\mathrm{PnO}$, pontine reticular nucleus, oral; $\mathrm{PO}$, periolivary nucleus; Pr5, principal sensory trigeminal nucleus; RN, red nucleus; RRF, retrorubral field; SN, substantial nigra; Sol, solitary nucleus; Sp5C, spinal trigeminal nucleus, caudal; Sp5I, spinal trigeminal nucleus, interpolar; $\mathrm{Sp5O}$, spinal trigeminal nucleus, oral; $\mathrm{SpVe}$, spinal vestibular nucleus; SuG, superficial gray layer of the superior colliculus; th, trigeminothalamic tract; Tz, nucleus of the trapezoid body; VC, ventral cochlear nucleus. Scale bar, $5 \mathrm{~mm}$.

An increase in labeling was seen at this time and higher-order structures that comprise thirdorder neurons were revealed. Labeled neurons in the medullary and reticular nuclei became more numerous bilaterally. The nucleus of the trapezoid body and the medial and lateral periolivary nuclei showed an increase in labeling and remained mostly ipsilateral. In addition, PRV-labeled neurons were seen in the medial ventral periolivary nucleus. The spinal and principal sensory trigeminal nucleus showed an increase in labeling and a few labeled neurons were seen on the contralateral side. A few labeled neurons were found in the ipsilateral motor trigeminal nucleus. The dorsal cochlear nucleus was labeled bilaterally and also some labeled neurons were seen in the ventral cochlear nucleus ipsilaterally. The parabrachial and Köllikerfuse nucleus showed a small increase in labeling and only a few neurons were seen on the contralateral side. Labeling in the medial vestibular nucleus became more dense and bilateral. In addition, PRV-labeled neurons were found in the superior and lateral vestibular nuclei bilaterally. PRV-labeled neurons became more numerous in the contralateral RN and the pararubral nucleus and a few labeled cells were seen on the ipsilateral side of these nuclei at this time (Figure $11 \mathrm{~A}$ ). Also, PRV-labeled neurons were found in the interstitial nucleus of Cajal (Figure 11 A) and the nucleus of Darkschewitsch bilaterally, with ipsilateral predominance. Labeled neurons were also found in the oculomotor nucleus, supraoculomotor nucleus (Figure 11 A) bilaterally and periaqueductal gray (Figure 11 B) mostly ipsilaterally. Some PRV-labeled 
neurons were also seen in the lateral lemniscus, mainly ipsilaterally (Figure 11 B). More importantly, the first labeled neurons in the DCN were seen at this time in the dorsolateral AIN and rostral fastigial nucleus ipsilaterally (Figure 11 C and D). Importantly, PRV-labeled neurons found in the AIN 41/2 days after PRV injection were located in a region that has been extensively implicated in EBC. In addition, electrical stimulation of dorsolateral AIN neurons can generate blink responses (Lavond et al., 1984; McCormick and Thompson, 1984a; Steinmetz et al., 1992). The fastigial nucleus projects primarily to vestibular and reticular nuclei and its rostral part is involved in vestibulospinal control, including regulation of muscle tone (Gardner and Fuchs, 1975; Buttner et al., 1991; Siebold et al., 1997). A group composite of PRV-labeled neurons 41/2 days after PRV injection is represented in Figure 12.

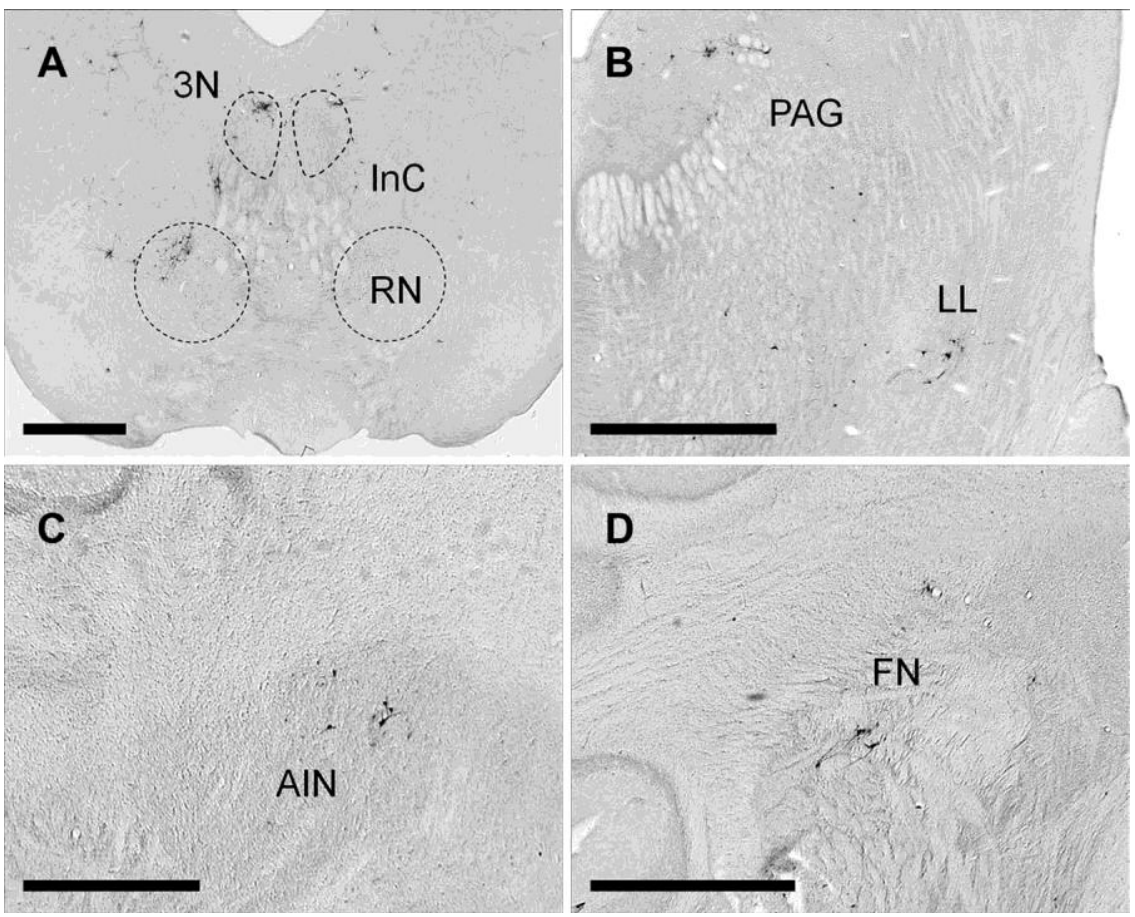

Figure 11. Examples of PRV-labeled premotor neurons $4 \frac{1}{2} 2$ days after PRV injection into the OOM. A-D. A shows PRV-labeled neurons in the dorsolateral red nucleus $(\mathrm{RN})$ and pararubral nucleus contralaterally. The oculomotor nucleus ( $3 \mathrm{~N})$, interstitial nucleus of Cajal ( $\mathrm{InC})$, and supraoculomotor periaqueductal gray are labeled bilaterally. B shows PRV-labeled neurons in the lateral lemniscus (LL) and periaqueductal gray (PAG) ipsilaterally. C shows a few PRV-labeled neurons in the dorsolateral AIN ipsilaterally. D shows PRV-labeled neurons in the rostral fastigial nucleus (FN) ipsilaterally. Scale bars: $1 \mathrm{~mm}$. 


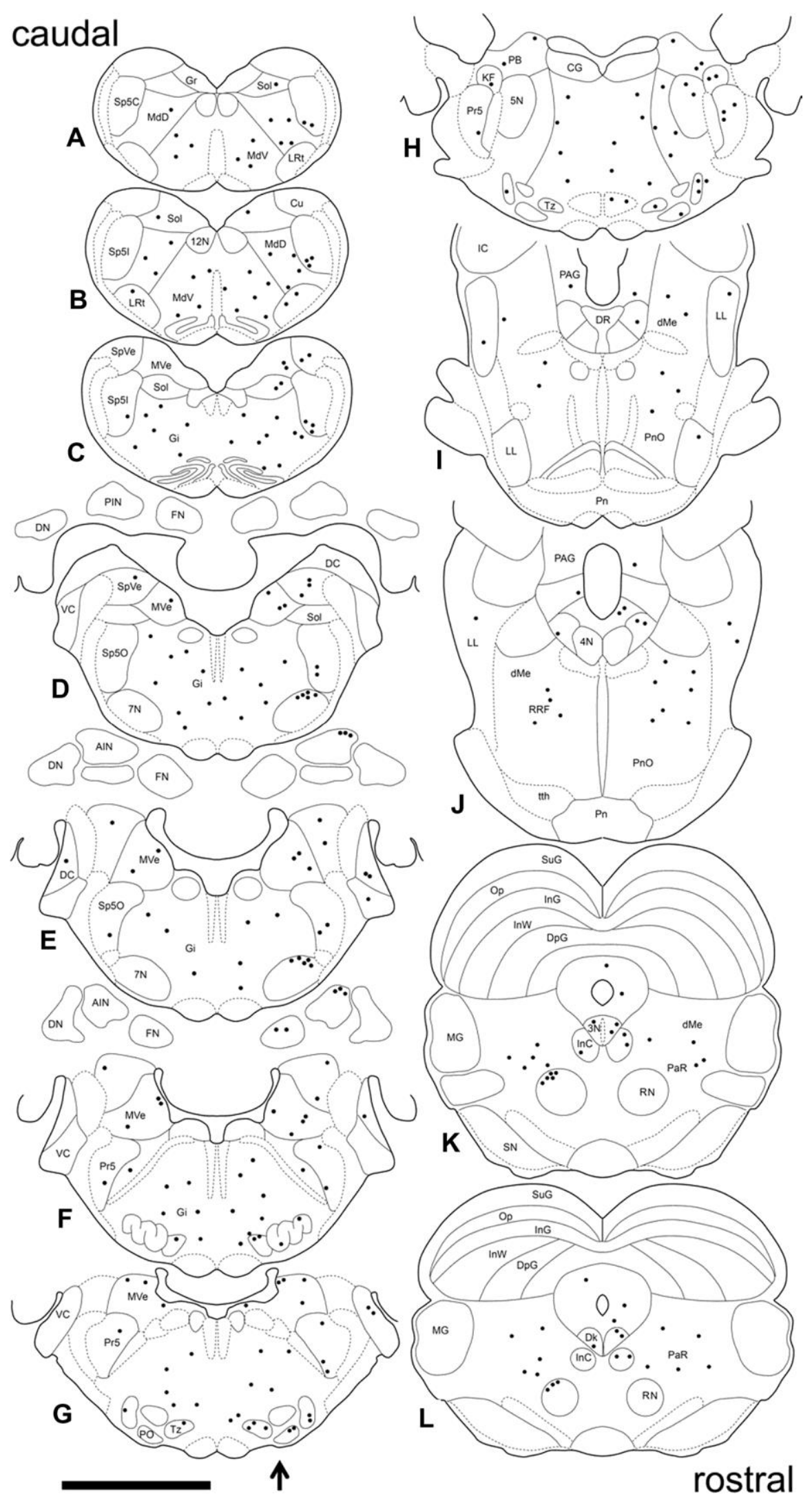


Figure 12. Distribution of PRV-labeled neurons 41/2 days after PRV injection into the OOM. A composite of the location of PRV-labeled neurons found across four animals plotted in sequential sections to best represent the pattern of PRV labeling. Sections are arranged caudorostrally (A-L). The arrow indicates the side of PRV injection. Each dot represents one PRV-labeled neuron. See Figure 3 for abbreviations. Scale bar, $5 \mathrm{~mm}$.

Five days after PRV injection, an increase in PRV-labeled neurons was seen throughout. In addition to previously labeled regions, labeled neurons were found in the cuneate (Figure 13 A), and gracile nucleus bilaterally. Labeled neurons were seen in the superficial gray and optic nerve layer of the contralateral superior colliculus (Figure 13 B) (McCormick et al., 1983; Halverson et al., 2009). An increase in labeling was seen in the pararubral nucleus, the deep mesencephalic nucleus and periaqueductal gray bilaterally (Figure $13 \mathrm{C}$ ). A few labeled neurons were seen in the substantia nigra, mainly ipsilaterally. At this time, an increase in labeling was seen in the DCN. Labeled neurons were found in specific regions of the fastigial, interpositus and dentate nuclei bilaterally with ipsilateral predominance.

Only two discrete regions in the ipsilateral cerebellar cortex showed PRV-labeled Purkinje cells at this time. The first region corresponds to lobule HVI in the longitudinal zone $\mathrm{C} 3$ (Figure 13 D and F). Interestingly, HVI has been extensively implicated in delay EBC (Yeo et al., 1985b; Gould and Steinmetz, 1996; Schreurs et al., 1997; Schreurs et al., 1998; Jirenhed et al., 2007; Kellett et al., 2010). Purkinje cells in HVI can generate a blink response and can be activated by periocular stimulation (Berthier and Moore, 1986; Hesslow, 1994a). In addition, these Purkinje cells receive inputs from climbing fibers that also send collaterals to the AIN (Yeo et al., 1985b; Pijpers et al., 2005; Sugihara and Shinoda, 2007). The location of PRV-labeled Purkinje cells was determined by careful analysis of their location using three different rabbit brain atlases (Girgis and Shih-Chang, 1981; McBride and Klemm, 1968; Shek et al., 1986) and publications by Ramnani et al. (1996), Kellet et al. (2010), and Vogel et al. (2009). PRV-labeled Purkinje cells were located in a small region of HVI. This region corresponded to the dorsolateral posterior portion of HVI, adjacent to the ventromedial region of the paramedian lobule. Labeled Purkinje cells were found in the same coronal plane as the anterior interpositus nucleus and located -3.0 through $-4.0 \mathrm{~mm}$ relative to lambda based on the co-ordinates used by (Ramnani and Yeo, 1996; Kellett et al., 2010), and $0.0 \mathrm{~mm}$ through $1.0 \mathrm{~mm}$ anterior to lambda based on the coordinates used by (Vogel et al., 2009). Based on their direct connection to the AIN and their 
topographical and anatomical location labeled Purkinje cells corresponded to longitudinal zone C3. Further comparison with the Sanchez et al. (2002) study of the parasagittal compartmentalization of the rabbit cerebellar cortex revealed by the expression of zebrin II +/stripes, shows that PRV-labeled Purkinje cells were located between zebrin II +/- stripes p4+ and p5+ of HVI, and most probably in p4- . The pattern of zebrin II +/- stripes expression is highly reproducible between individuals and conserved across species (Brochu et al., 1990; Sanchez et al., 2002; Sillitoe et al., 2005). Purkinje cells located in zebrin II +/- stripe p4- have been shown to project to the anterior interpositus in mice and rats (Sugihara and Quy, 2007; Sugihara and Shinoda, 2007; Sugihara, 2011) and in the rabbit it may correspond to p4b- (Sanchez et al., 2002). However in the present study, the correspondence of PRV-labeled Purkinje cells with specific zebrin II +/- stripe can only be inferred based on their anatomical location.

The second region corresponds to the rostral part of lobule I in the longitudinal zone A (Figure 13 E). Purkinje cells located in lobule I, longitudinal zone A have been shown to project to the fastigial nucleus (Armstrong and Schild, 1978). Labeled Purkinje cells in lobule I may be part of a cortico-nuclear-vestibular pathway involved in controlling the tone and position of the eyelid with respect to the eye-ball and may serve as a muscle position and motion detector to modulate the coordination of different muscles during eyelid movements (Shaikh et al., 2005; Brooks and Cullen, 2009).The distribution of labeled Purkinje cells seems to delineate two distinct cerebellar OOM premotor pathways. The first involves HVI, the AIN, the RN and the facial nucleus, whereas the second may involve lobule I, the fastigial nucleus, the vestibular nucleus and the facial nucleus. Taken together, these data show a comprehensive map of the premotor pathway of the $O O M$ and important structures involved in the eyeblink response. 

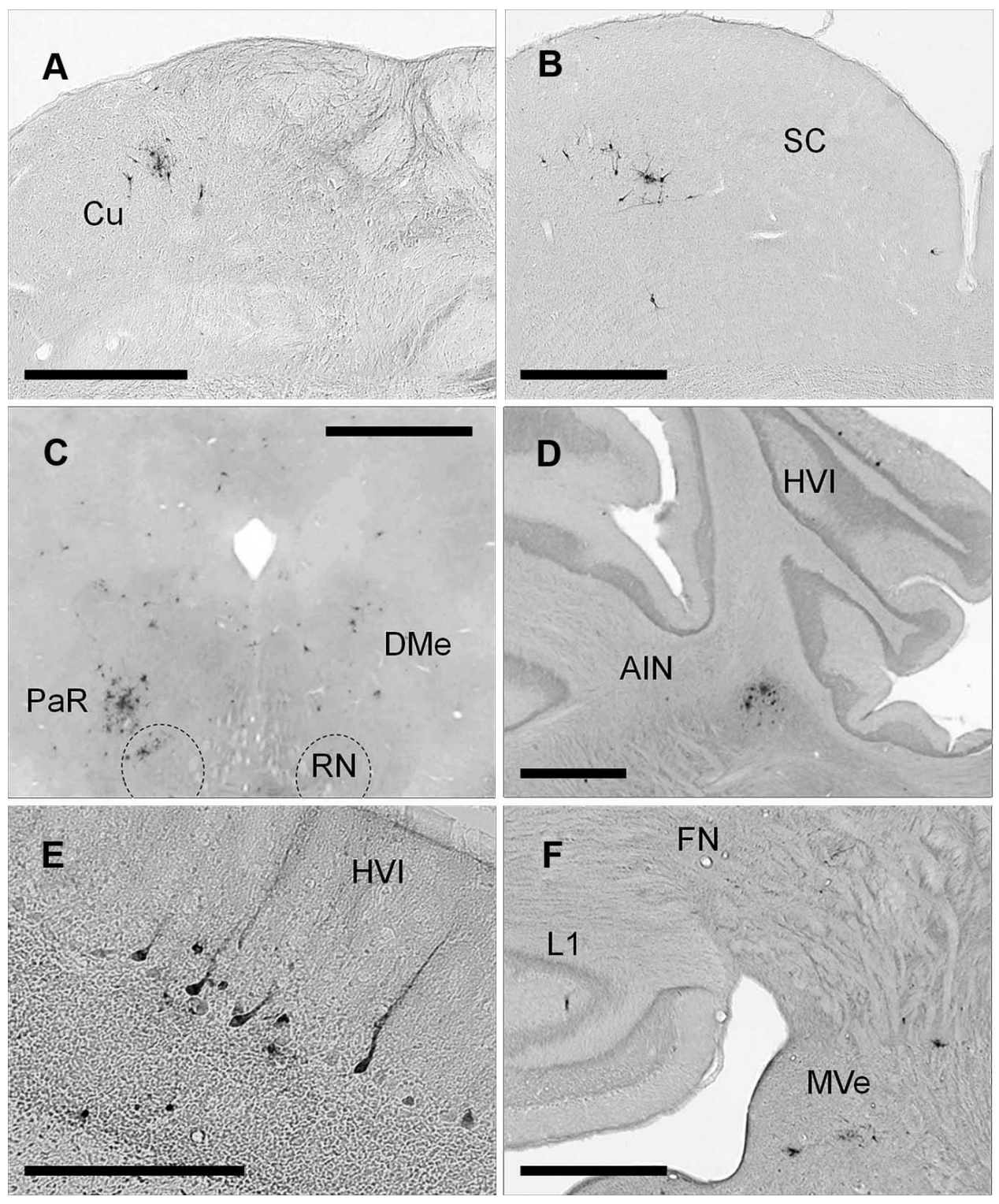

Figure 13. Examples of PRV-labeled eyeblink premotor neurons 5 days after PRV injection into OOM. A shows PRV-labeled neurons in the $\mathrm{Cu}$ (cuneate nucleus) ipsilaterally. B shows PRV-labeled neurons in the superior colliculus (SC) contralaterally. C shows PRV labeling in the red nucleus (RN) and pararubral nucleus (PaR) mainly contralaterally and deep mesencephalic nucleus (DMe) bilaterally. D shows PRV-labeled neurons in HVI longitudinal zone C-3 ipsilaterally; note AIN PRV-labeled neurons in the same section. E shows a group of PRV-labeled Purkinje cells in HVI zone C-3 ipsilaterally. F shows PRV-labeled cerebellar lobule I (L1) longitudinal zone A ipsilaterally, note PRV-labeling in the rostral fastigial nucleus (FN), lateral and medial vestibular nucleus (MVe) ipsilaterally. Scale bars: AD, F, 1 mm; E, 200um. 


\section{$P R V$ rate of spread}

We found that PRV consistently traced the same pathway in a time-dependent manner across all subjects. PRV axonal transport and the replication cycle seem to be dependent on viral titer, neuronal activity, strength of functional synapses and species. Axonal transport has been estimated to occur at rates of $8-12 \mathrm{~mm} / \mathrm{hr}$ in vivo (Tomishima et al., 2001) and on average, the virus's complete replication cycle in the nervous system is about 6-16 hrs to the point of egress (Whealy et al., 1988; Ugolini, 2011). It has been suggested that postsynaptic neurons that innervate the soma and make numerous synapses onto an infected cell may become infected sooner than those making distal and fewer contacts (Song et al., 2005). Likewise, neuronal activity seems to influence the rate of PRV labeling and this is supported by our data. Neurons in the RN that project to the motor facial nucleus are phasically active (Gibson et al., 1985) whereas DCN neurons are tonically active. PRV labeled the RN (second-order neurons) in 24 hrs and the DCN (third-order neurons) in $12 \mathrm{hrs,} \mathrm{even} \mathrm{though} \mathrm{PRV} \mathrm{had} \mathrm{to} \mathrm{travel} \mathrm{the} \mathrm{same} \mathrm{distance.}$ In addition, PRV labeled fourth-order Purkinje cells (that are also tonically active) in $12 \mathrm{hrs,}$ suggesting that PRV's time-dependent spread correlates with neuronal activity rather than distance traveled and it could be of particular importance for delineating functionally relevant pathways.

\section{Distribution of premotor neurons in the DCN}

To further characterize the distribution of DCN premotor neurons, the location of $4 \frac{1}{2}$ and 5 days PRV-labeled cells was plotted in a map of coronal sections. The first PRV-labeled neurons seen in the DCN at $4 \frac{1}{2}$ days were consistent with third-order premotor neurons and found in the dorsolateral AIN and rostral FN exclusively on the ipsilateral side at this time (Figure 14 A). These two distinct areas may hold particular importance in the cerebellar premotor pathway of the OOM. An increase in PRV labeling was seen bilaterally in the DCN 5 days after injection (Figure 14 B). At this time, groups of large and small PRV-labeled neurons were seen in all three nuclei consistent with third and fourth-order neurons. The distributions of these neurons could be of importance in elucidating the somatotopic organization of the OOM in all three nuclei. 


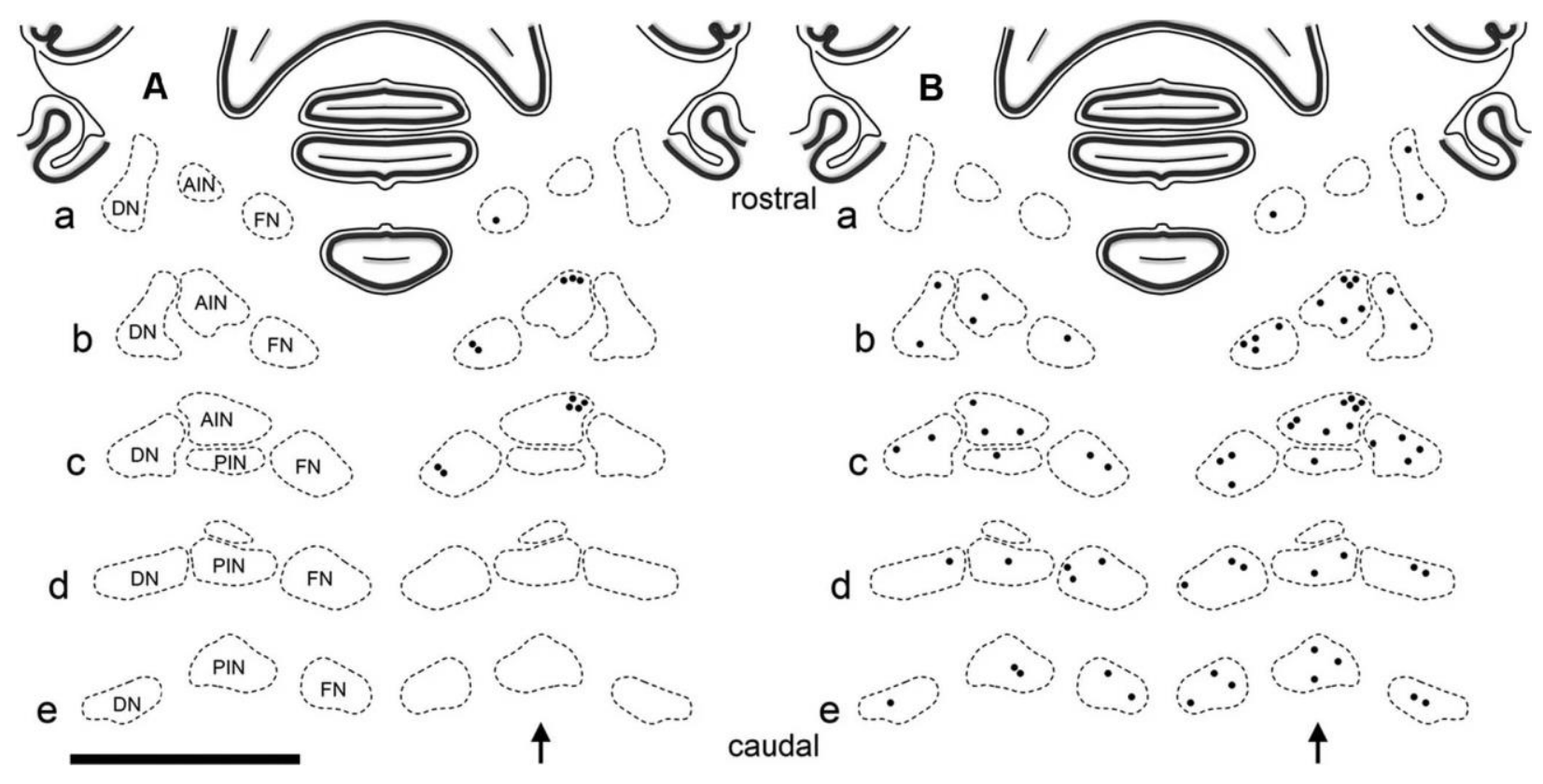

Figure 14. Distribution of PRV-labeled eyeblink premotor neurons in the deep cerebellar nuclei.

Distribution of PRV-labeled premotor neurons in the DCN 4.5 and 5d after PRV injection. Sections are arranged rostrocaudally (a-e). Note each dot represents a group of 2-5 PRV-labeled neurons of a typical subject. A shows the distribution of PRV-labeled neurons in the rostral fastigial nucleus (FN) and dorsolateral AIN that are found exclusively on the ipsilateral side 4.5d after PRV-injection. B shows the distribution of PRV-labeled neurons in the FN, AIN, posterior interpositus nucleus (PIN), and dentate nucleus (DN) that are found bilaterally with ipsilateral predominance 5d after PRV injection. Scale bar, $5 \mathrm{~mm}$.

\section{Neurotransmitter immunoreactivity in the AIN}

The large and small PRV-labeled neurons seen in the ipsilateral AIN 5 days after injection (Figure 14 B) were presumably third-order glutamatergic projection neurons and fourth-order interneurons. To further investigate this local network, we used glutamate, GABA and glycine immunoreactivity (ir) to label these neurons. All sections containing the ipsilateral DCN were immunolabeled and as a control, the cerebellar cortex was examined in each section to confirm the specificity of neurotransmitter-ir. Figure 15 shows glutamate-, GABA- and glycine-ir neurons in the cerebellar cortex. Granule cells and their numerous axon terminals extending through the molecular layer were immunoreactive for glutamate. Purkinje cells, small basket and stellate cells in the molecular layer, and Golgi cells in the granule cell layer were immunoreactive for GABA. Neurons immunoreactive for glycine include Lugaro cells in the 
Purkinje cell layer, and Golgi cells in the granule cell layer. Co-localization of GABA- and glycine-ir can be seen in some Golgi and Lugaro cells in the granule cell layer, known to be GABA/glycinergic neurons (Crook et al., 2006; Simat et al., 2007).

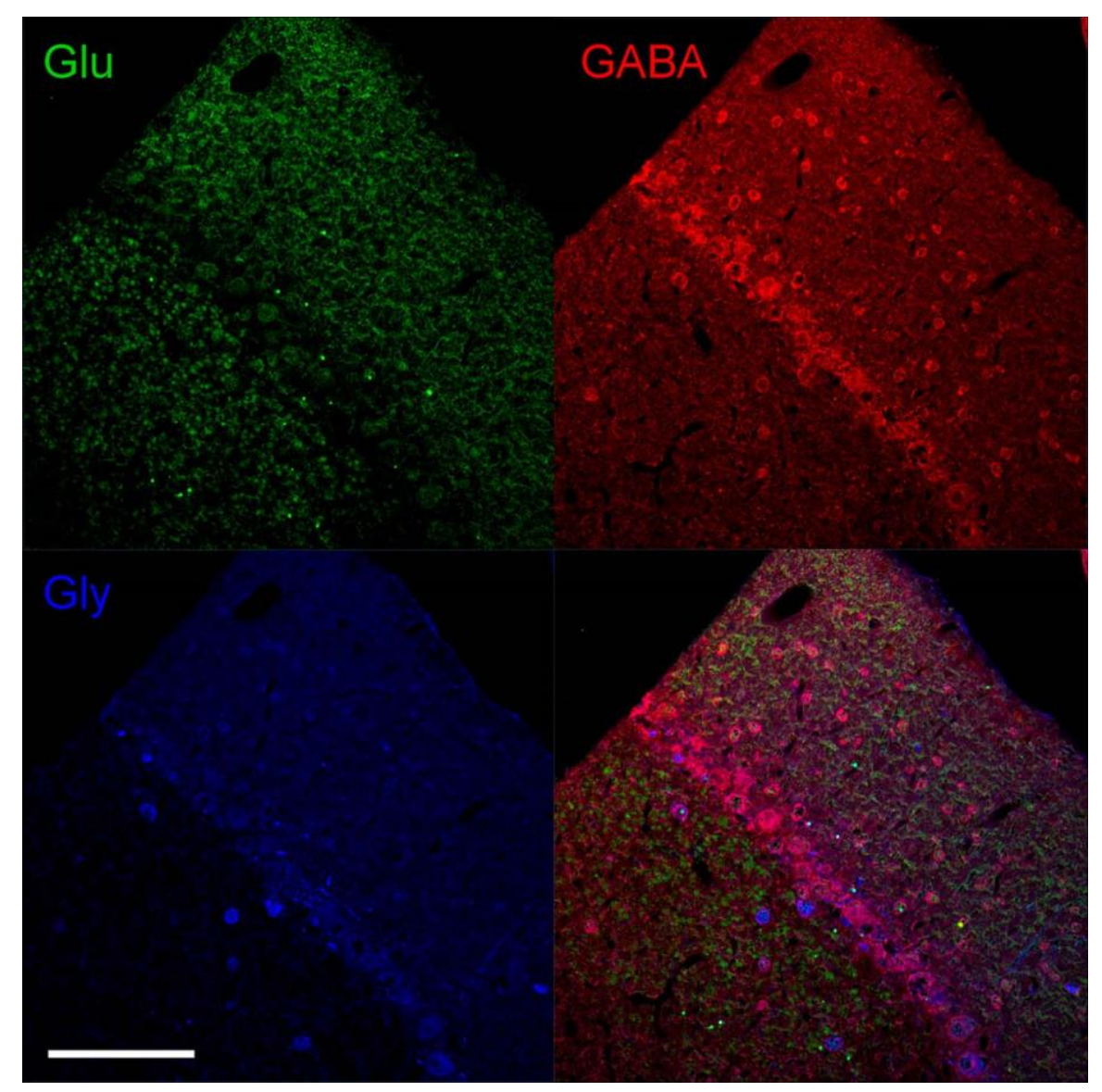

Figure 15. Glutamate, GABA, and glycine immunoreactivity in the cerebellar cortex. Glutamate-immunoreactive granule cells and their numerous axon terminals extending through the molecular layer are seen in the top left panel. GABA-immunoreactive neurons including Purkinje cells, basket, and stellate cells in the molecular layer, and Golgi cells in the granule cell layer are shown in the top right panel. Glycine-immunoreactive neurons in the cerebellar cortex including Lugaro cells in the Purkinje cell layer, and Golgi cells in the granule cell layer are shown in the bottom left panel. A merged image of glutamate-, GABA-, and glycine-immunoreactive neurons is shown in the bottom right panel. Colocalization of GABA and glycine-immunoreactive neurons can be seen in some Golgi and Lugaro cells. Glu, Glutamate; Gly, glycine. Scale bar, $200 \mu \mathrm{m}$. 
Glutamate-, GABA- and glycine-ir in the DCN including the AIN, DN, and granule cell layer of the cerebellar cortex is shown in Figure 16. Large neurons in the DCN and small granule cells in the cerebellar cortex are glutamate-ir. Small neurons and numerous terminals in the DCN are GABA-ir. In addition, several Golgi cells in the granule cell layer in the same section are also GABA-ir. Glycine-ir neurons are small and evenly distributed in the DCN.

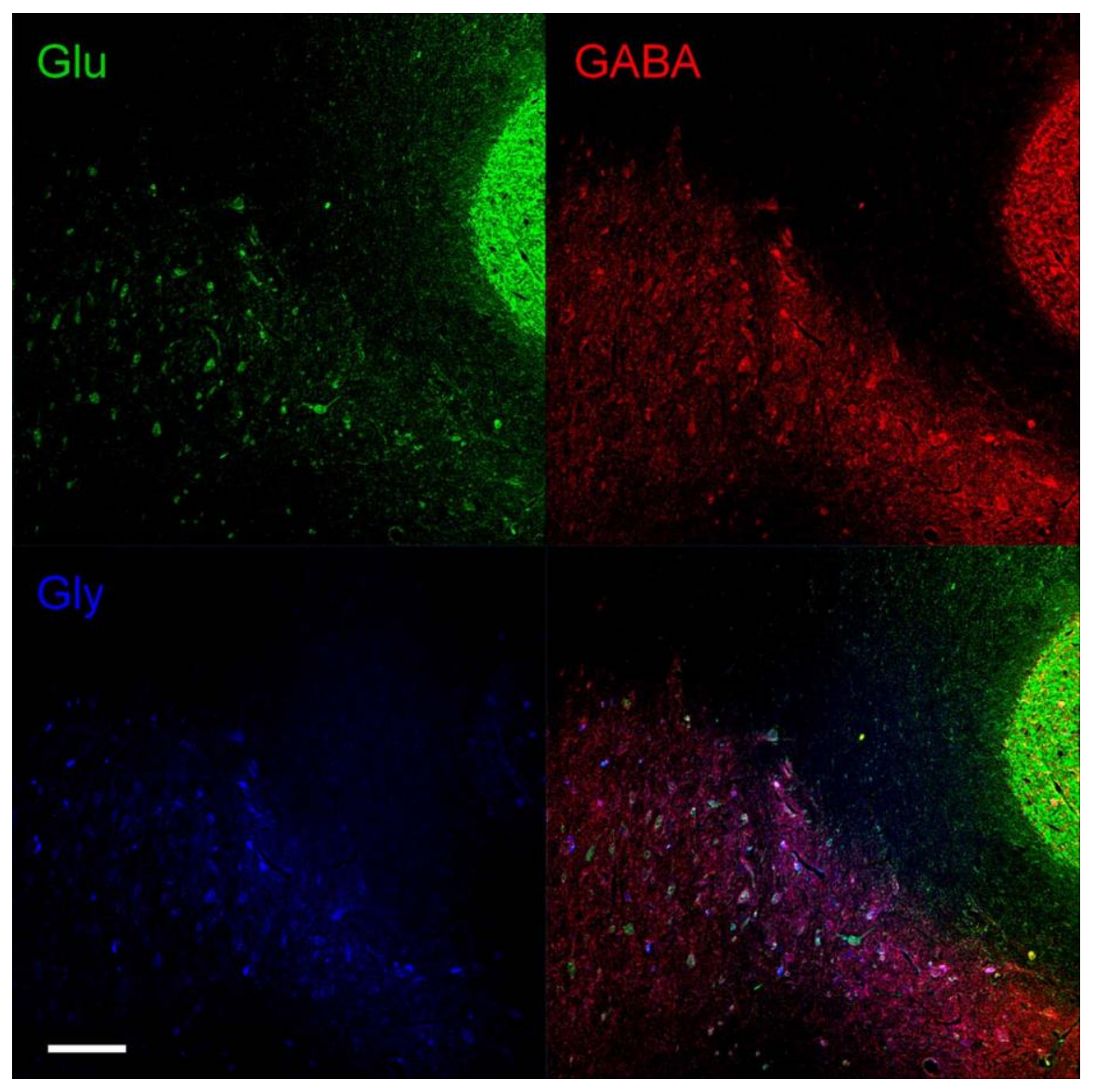

Figure 16. Glutamate, GABA, and glycine immunoreactivity in the deep cerebellar nuclei. Glutamate, GABA, and glycine immunoreactivity in the DCN and cerebellar cortex. A section that contains the dorsolateral AIN, dorsal dentate nucleus (DN), and the granule cell layer of the cerebellar cortex is shown. Glutamate-immunoreactive neurons are shown in the top left panel. Note large glutamate-immunoreactive neurons in the DCN and numerous granule cells in the cerebellar cortex. GABA-immunoreactive neurons are shown in the top right panel. Note smaller GABA-immunoreactive neurons and numerous terminals in the DCN and several Golgi cells in the granule cell layer. Glycine-immunoreactive neurons are shown in the bottom left panel. Note small distinctive glycine-immunoreactive neurons evenly distributed in the DCN. A merged image of glutamate, GABA, and glycine-immunoreactive neurons is shown in the bottom right panel. Glu, Glutamate; Gly, glycine. Scale bar, $400 \mu \mathrm{m}$. 


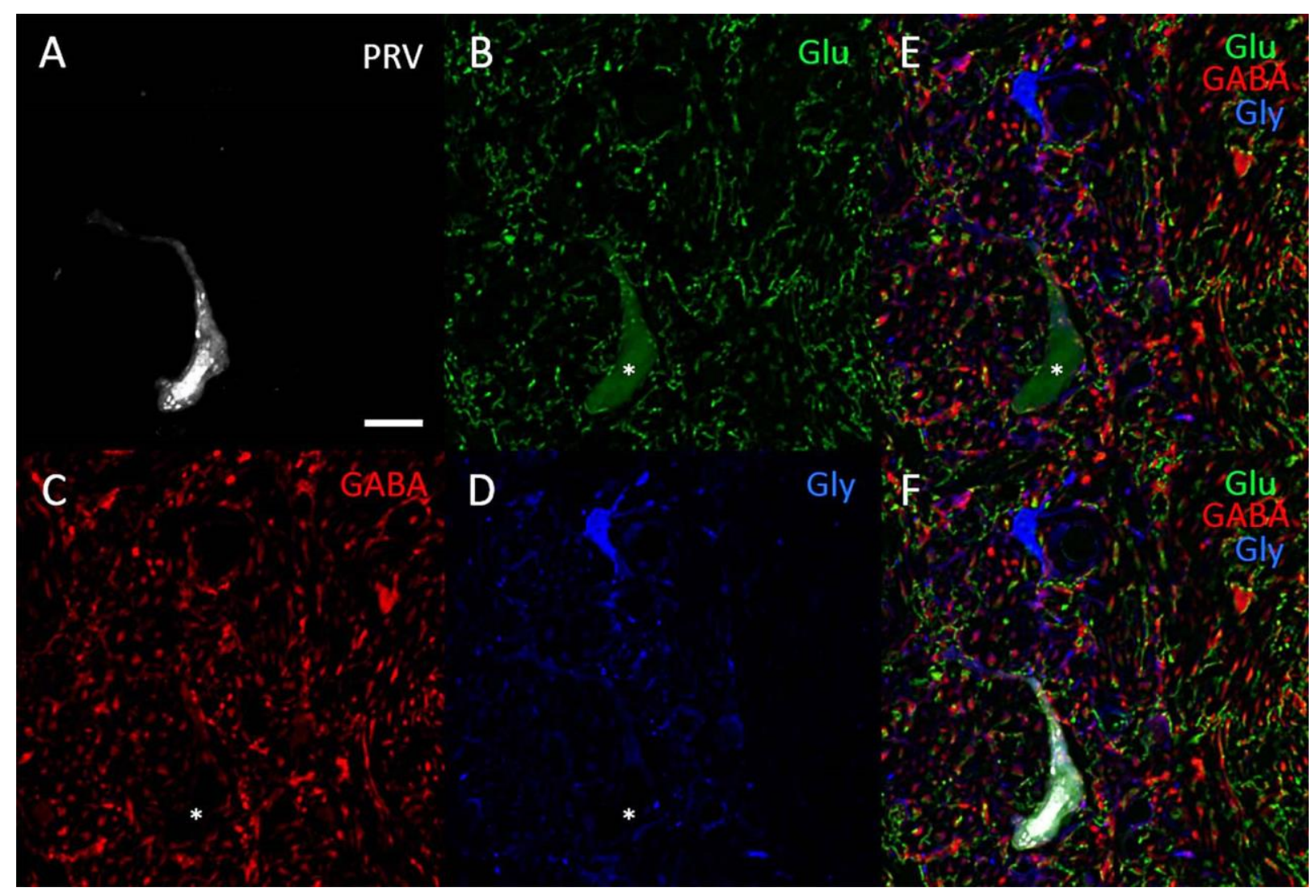

Figure 17. Eyeblink premotor neuron in the AIN immunoreactive for glutamate.

Glutamate-immunoreactive (ir) PRV-labeled neuron in the AIN $4.5 \mathrm{~d}$ after injection into the OOM. An eyeblink premotor AIN neuron labeled with PRV is shown in A. Glutamate-ir is shown in B. Note a large glutamate-ir neuron indicated by the asterisk. GABA-ir is shown in C. Numerous GABA-ir terminals are evident apposed to different neuronal processes and glutamate-ir soma, also note a small GABA-ir neuron (C). Glycine-ir is shown in D. Note a small interneuron that is exclusively glycine-ir. A few glycine-ir terminals apposing dendrites and somas of glutamate-ir and GABA-ir neurons are shown. A merged image of glutamate-, GABA-, and glycine immunoreactivity is shown in E. A merged image of D and PRV is shown in F. Note that the PRV-labeled neuron colocalizes with a large glutamate-immunoreactive neuron. Glu, Glutamate; Gly, glycine. Scale bar, $20 \mu \mathrm{m}$.

In addition, glutamate was found to co-localize with most GABA-ir neurons to various degrees.

Because glutamate is a precursor of GABA, it is present in GABAergic cells as a function of their metabolic state (Ottersen and Storm-Mathisen, 1984; Sultan et al., 2002). Also, glutamate labeling was never found in purely glycine-ir neurons. However, GABA/glycine-ir neurons showed glutamate labeling to various degrees. Consequently, glutamate-ir neurons were 
classified as glutamatergic only when they did not co-localize with other neurotransmitters. An example of a 41/2-day PRV-labeled neuron immunoreactive for glutamate is shown in Figure 17. PRV-labeled neurons immunoreactive for glutamate were large $(29 \mu \mathrm{m} \pm 8)$ and an average of $20 \pm 7(n=4)$ were found in the AIN 41/2 days after injection.

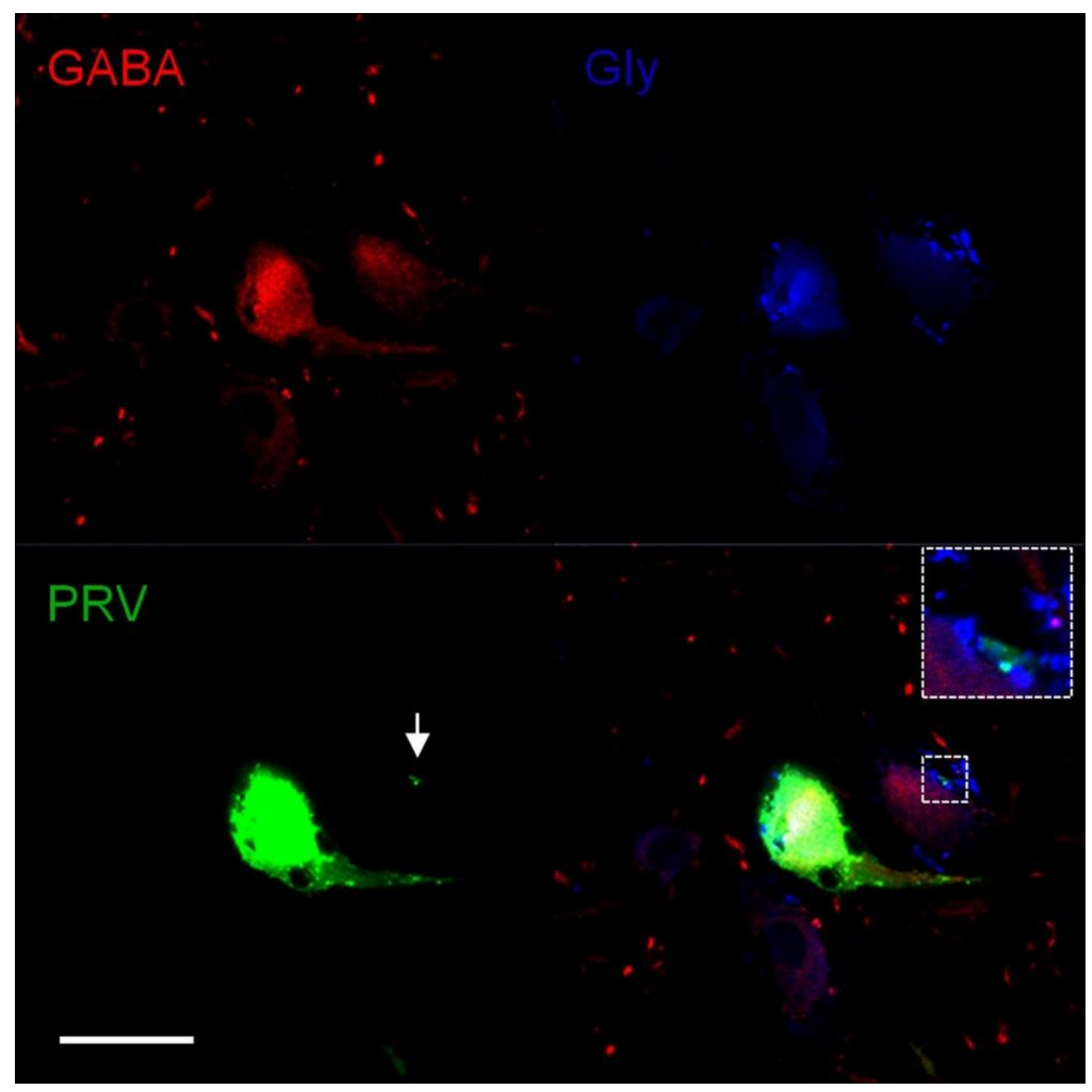

Figure 18. Anterior interpositus interneuron labeled with PRV.

GABA and glycine immunoreactive interneuron labeled with PRV. GABA immunoreactivity is shown in the top left panel. Note two GABA-immunoreactive interneurons and numerous GABA-immunoreactive terminals. Glycine immunoreactivity is shown in the top right panel. Note two Glycine-immunoreactive interneurons and a few and densely packed Glycineimmunoreactive terminals. PRV-labeled fourth-order interneuron and its PRV-labeled terminal are shown in the bottom left panel. A merged image of GABA immunoreactivity, glycine immunoreactivity, and PRV is shown in the bottom right panel. Note that the PRV-labeled interneuron is immunoreactive for both GABA and glycine and its PRV-labeled terminal is immunoreactive solely for glycine. Glu, Glutamate; Gly, glycine. Scale bar $20 \mu \mathrm{m}$. 
Next, characterization of PRV-labeled neurons 5 days after injection revealed an average of $34 \pm 9$ $(n=4)$ glutamate-ir neurons and an average of $66 \pm 12$ interneurons immunoreactive for GABA and/or glycine. The interneurons were immunoreactive for GABA (30\%), glycine (18\%), or for both GABA and glycine (52\%). The average soma diameter for PRV-labeled interneurons immunoreactive for GABA was $16 \mu \mathrm{m} \pm 4 \mu \mathrm{m}$, for glycine was $15 \mu \mathrm{m} \pm 4 \mu \mathrm{m}$, and for GABA/glycine was $17 \mu \mathrm{m} \pm 5 \mu \mathrm{m}$. An example of a PRV-labeled interneuron 5 days after injection is shown in Figure 18. In this case, a PRV-labeled interneuron is immunoreactive for both GABA and glycine. We were able to follow the PRV-labeled axon of this neuron and found its terminal apposed to an adjacent interneuron that was also GABA/glycine-ir.

Interestingly, the terminal of the PRV-labeled interneuron was glycine-ir only, suggesting that mixed GABA/glycinergic interneurons in the DCN can selectively release either neurotransmitter from their terminals. Differential neurotransmitter release from mix GABA/glycinergic neurons has been previously hypothesized (Dumoulin et al., 2001; Dugue et al., 2005; Dufour et al., 2010; Benarroch, 2011).

\section{Discussion}

The present study shows the premotor pathway of the rabbit $O O M$ using PRV as a retrograde transneuronal tracer. Relevant structures involved in the generation or modulation of the rabbit eyeblink response were identified and provide anatomical validation of a direct premotor network that involves the dorsolateral AIN and HVI of the cerebellar cortex, which have been extensively implicated in EBC. Of particular importance, classification of fourth-order premotor interneurons based on their neurotransmitter immunoreactivity revealed three different types of inhibitory interneurons that are part of the $O O M$ premotor pathway and form a functional network within the AIN for the modulation of motor commands. 


\section{Premotor pathway of the rabbit OOM}

PRV revealed $O O M$ premotor structures involved in spontaneous, reflex and conditioned eyeblink responses. The main function of the eyelid and the blink response is to protect the eye and this response can be elicited by different sensory modalities. Second- and third-order premotor neurons were found in different reticular nuclei, sensory trigeminal, auditory, vestibular and motor structures. Important for EBC are sites that integrate information from the conditioned and unconditioned stimulus (eg. tone and airpuff). The eyeblink premotor pathways revealed here constitute possible locations for integration and storage of conditioned responses. For a detail discussion on the OOM premotor pathways see (Morcuende et al., 2002; DelgadoGarcia et al., 2003).

\section{Cerebellar cortex}

There is an ongoing controversy about which lobule in the cerebellar cortex control conditioned eyeblinks. Some studies in the rabbit using permanent lesions, infusions of AMPA receptor antagonists or single and multi unit recordings identified HVI to be involved in EBC (Berthier and Moore, 1986; Gould and Steinmetz, 1996; Attwell et al., 2001). More recent studies using lesions, single unit recordings or infusions of lidocaine and picrotoxin identified the anterior lobule HIV and HV to be involved in EBC (Garcia et al., 1999; Green and Steinmetz, 2005; Kalmbach et al., 2010). However, the functional organization of the cerebellar cortex is consistent with longitudinal zones that span through multiple lobules, and the eyelid controlling region in the rabbit may not be confined to one lobule.

There are a number of regions in the cerebellar cortex that have been found to receive climbing fiber input from periocular stimulation, exhibit eyeblink related activity patterns and project to the AIN. In addition a new microzone has been identified in the rabbit located in the ventral portion of HVI (Mostofi et al., 2010). It is conceivable that different eyeblink microzones encode different types of information including time, space, intensity, and duration and that 
together these microzones convey this information to their target nuclear cells which in turn elicit a motor command.

To date, it is not clear how Purkinje cell activity controls eyelid movements. It has been hypothesized that a pause in inhibitory, tonically active Purkinje cells allows their target nuclear cells to produce the necessary firing activation required to generate a motor command. Another possibility is that a burst in Purkinje cell activity produces strong inhibition (hyperpolarization) of its target nuclear cell that is followed by rebound depolarization resulting in a transient increase in firing rate generating a motor command (Pugh and Raman, 2006; Alvina et al., 2008; Molineux et al., 2008). Interestingly, recordings from eyeblink microzones show that some Purkinje cells increase their firing rate while others decrease firing during performance of a conditioned eyeblink response.

\section{The microcircuit of the AIN}

All information processed in the cerebellum is integrated in the DCN where motor commands are generated and carried out by projection neurons. Despite its importance, little is known about the components that constitute this local network and their interconnections. The possible interactions among interneurons in the DCN and their role in learning are largely unknown. The AIN has been extensive implicated in EBC and many believe that synaptic changes that allow a conditioned response to emerge reside in this nucleus. Lesion and inactivation studies show that the AIN is critical for the formation of conditioned responses (Lavond et al., 1984; Steinmetz et al., 1992; Krupa and Thompson, 1997). Single unit recording studies in the AIN show that some neurons change their pattern of activation during learning. These neurons increase their firing rate during the execution of conditioned responses in a pattern that precedes and predicts the performance of a conditioned response (McCormick et al., 1983; McCormick and Thompson, 1984b; Berthier and Moore, 1990; Tracy et al., 2001). In vitro electrophysiological studies in the interpositus nucleus show that large neurons can undergo different types of synaptic plasticity including LTP and LTD (Pugh and Raman, 2006). 
Most of our understanding on the properties of nuclear cells derives from a single neuronal type, the large excitatory projection neuron, mainly because identification of different nuclear cells was based solely in soma size. Recently, the electrophysiological properties of DCN interneurons have been possible in transgenic mice expressing GAD-eGFP or GlyT2-eGFP (Uusisaari and Knopfel, 2011). However, little is known about the synaptic connections and interactions among the different neuronal types. Our results show three types of interneurons that are directly connected to large glutamatergic projection neurons and possibly involved in modulating their output. The DCN is a complex structure and its different components and interactions contribute to the integration of input from the cerebellar cortex and sensory information to generate a final cerebellar output.

Here we show a local network within the anterior interpositus consisting of a glutamatergic projection neuron and three types of inhibitory interneurons that are part of the $O O M$ premotor pathway. PRV-labeled fourth-order interneurons were classified as GABAergic, glycinergic or mixed GABA/glycinergic based on their neurotransmitter-ir. An example of such interconnectivity was found in a PRV-labeled mixed GABA/glycinergic interneuron in which we were able to follow the axon. The terminal of this interneuron was labeled with PRV and apposed the soma of another interneuron also immunoreactive for GABA and glycine that was not labeled with PRV, suggesting that these two interneurons contact different glutamatergic neurons. GABA and glycine are frequently co-expressed in the same cells and are co-released from the same terminals (Ottersen et al., 1988; Dumoulin et al., 2001; Dugue et al., 2005). It has been suggested that mixed GABA/glycinergic neurons can modulate the GABA/glycine ratio that is released from their terminals as these neurotransmitters have different effects on their postsynaptic targets (Benarroch, 2011). Interestingly, the synaptic terminal of the PRV-labeled mixed GABA/glycinergic interneuron was immunoreactive solely for glycine suggesting differential neurotransmitter release. Glycine has been implicated in different types of synaptic plasticity including long term potentiation (Martina et al., 2004) and is required for the activation of NMDA receptors (Johnson and Ascher, 1987) and suggests another possible mechanism of plasticity in the AIN. 


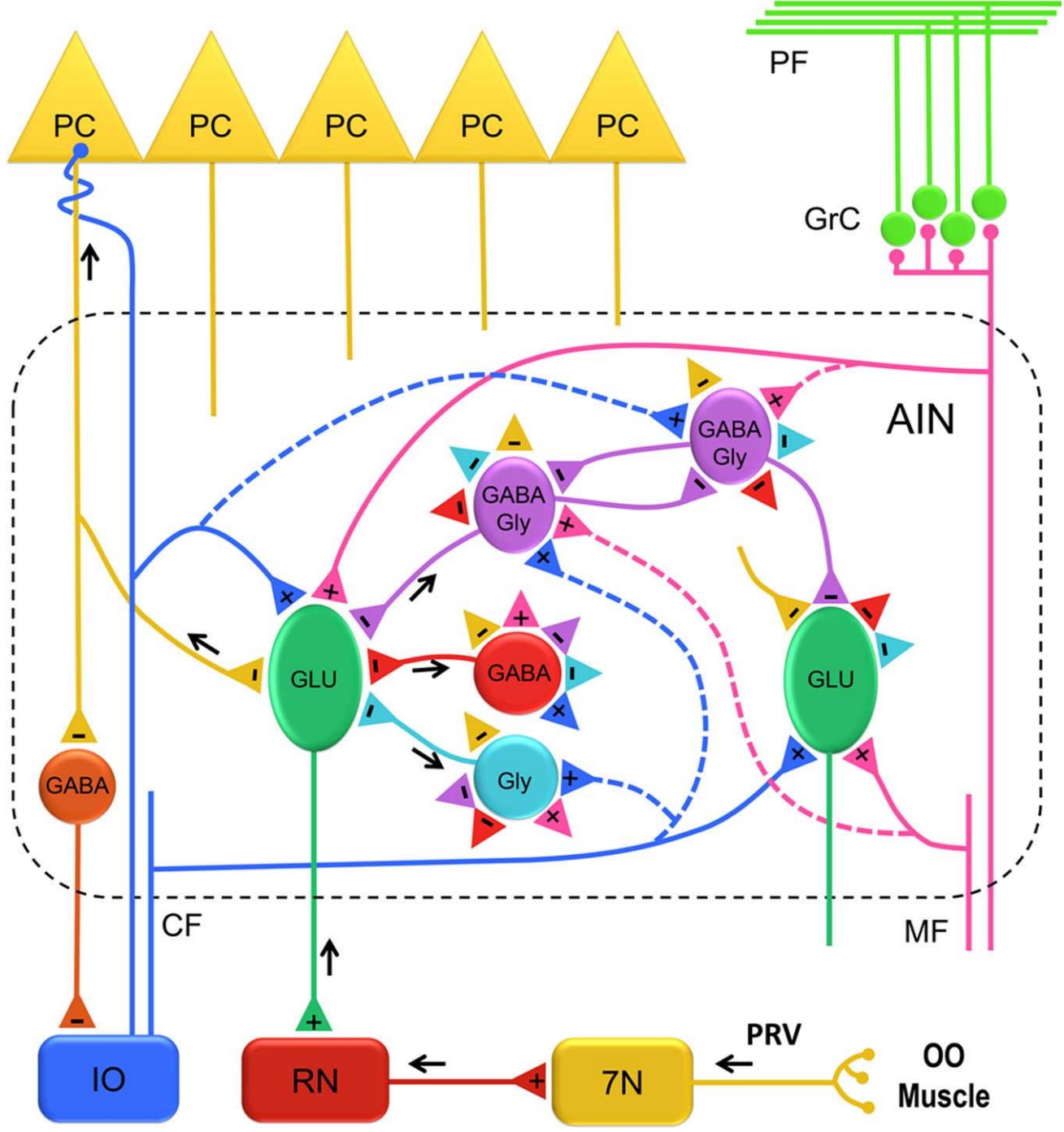

Figure 19. Organization of the anterior interpositus circuit.

A schematic representation of different neuronal populations and their connections in the AIN. The premotor pathway of the OOM is shown with black arrows that delineate the direction of PRV labeling. Two representative glutamatergic (GLU) projection neurons are shown. A PRVlabeled third-order GLU neuron projecting to the red nucleus $(\mathrm{RN})$ is depicted with a black arrow. Three types of PRV-labeled fourth-order interneurons are shown to make a direct connection onto the PRV-labeled GLU neuron. Possible connections among different AIN neurons are represented with excitatory $(+)$ and inhibitory (-) terminals (colored triangles). Excitatory input reaches the AIN by mossy fiber (MF) and climbing fiber (CF) collaterals that make connections on GLU neurons and possibly (dashed lines) on different interneurons. Inhibitory input to AIN neurons is provided by Purkinje cells (PC) and local interneurons. 7N, Facial nucleus; GrC, granule cell layer; IO, inferior olive; PF, parallel fibers. 
Based on the observed interconnectivity of interneurons described above and current knowledge of the AIN organization, a schematic diagram of possible interactions is depicted in Figure 19. The figure shows Purkinje cell inhibitory inputs as well as excitatory mossy fiber and climbing fiber collateral inputs arriving at large glutamatergic projection neurons. Also depicted are Purkinje cell inhibitory inputs and the presumed mossy fiber and climbing fiber collateral projections to interneurons that provide their only excitatory input and source of activation. Finally, the figure proposes inhibitory connections between interneurons. Examination of these connections allows one to speculate about the consequences of these possible interactions among and between interneurons and projection neurons. For example, although mossy fiber and climbing fiber collaterals activate a PRV-positive glutamatergic projection neuron, they also activate inhibitory interneurons that, in turn, inhibit adjacent PRV-negative projection neurons creating an inhibitory surround. In addition, the inhibitory interneuron contacted by these mossy

fiber and climbing fiber collaterals also synapses onto a second interneuron which, in turn, disinhibits the PRV-positive projection neuron. As a result of releasing inhibition, sensory inputs can increase the excitation of the PRV-positive projection neuron while indirectly inhibiting adjacent PRV-negative projection neurons. These interactions are modulated by Purkinje cell inhibition. One possible consequence of this combination of excitation and inhibition of projection neurons could be co-ordination of the OOM and levator palpebrae allowing closure of the eyelids (Sanchez-Campusano et al., 2012).

\section{Comparison with other transsynaptic studies of the OOM}

A study in mouse using PRV (Sun, 2012) and another in rat using rabies virus (Morcuende et al., 2002) have also attempted to delineate the eyeblink premotor circuit. Despite some similarities, there are significant differences between our results and those of Sun (2012). For example, although motoneurons were found in ipsilateral facial nucleus after PRV injection, Sun found labeling in ventral and dorsal RN bilaterally which is different from the ipsilateral labeling we found in the dorsolateral portion of the RN. Previous research has shown that only the contralateral RN projects directly to the facial nucleus (Takada et al., 1984; Holstege and Tan, 1988). This suggests Sun (2012) may have identified additional pathways not directly connected 
to the facial nucleus because she averaged across different time points and so, labeled cells representing different synaptic steps were combined. This seems to be true for the DCN and cerebellar cortex as well because Sun found extensive bilateral labeling of all three deep cerebellar nuclei as well as bilateral labeling throughout the cerebellar cortex. Thus, a direct premotor pathway from cerebellum to the facial nucleus may have been present in PRV-injected mice but was obscured by more indirect pathways.

An earlier study of the rat $O O M$ premotor pathway was performed using rabies virus (Morcuende et al., 2002) and correlates very closely with the rabbit $O O M$ premotor pathway identified using PRV, at least to the level of third-order premotor neurons. Morcuende et al. (2002) found rabies-labeled neurons in caudolateral AIN and dorsolateral hump of DCN, whereas in the rabbit, third-order neurons were located in dorsolateral AIN and the rostral fastigial nucleus. In addition, they found fourth-order labeled Purkinje cells in the vermis and paravermis zones $\mathrm{C} 1-\mathrm{C} 3$, whereas in the rabbit, fourth-order Purkinje cells were found exclusively in HVI zone C3 and Lobule I zone A. This difference may reflect anatomical or functional differences between species or variations in viral kinetics. 


\section{Chapter 4. Synaptic plasticity in eyeblink projection neurons of the AIN}

\section{Introduction}

The AIN is necessary for the formation and execution of CRs and evidence suggests that the learning engram of EBC may reside in this nucleus. Behavior is mediated by synaptically connected neuronal networks that are plastic and continuously changing in response to external and internal stimuli. However, the learning mechanism underlying the formation of CRs is largely unknown. Learning has been associated with a number of factors including intrinsic membrane excitability, synaptic plasticity, and changes in synaptic connectivity. Synapses are the functional unit of networks and can undergo different types of activity-dependent changes that can directly influence the transmission of signals and information. In addition, learning in the AIN has been associated with synapse formation. Indeed, inhibition of protein synthesis during EBC prevents the acquisition of CRs (Bracha et al., 1998). Protein synthesis is required for structural changes associated with synapse remodeling and synaptogenesis, suggesting that synapse formation may be necessary for the acquisition of CRs during EBC.

The soma of large nuclear neurons is covered by converging afferent inputs. The majority of somatic inputs originate from PCs, however they intermingle with other extracerebellar and intracerebellar afferent fibers that together enclose the soma of large nuclear neurons forming a dense pericellular plexus or nest (Matsushita and Iwahori, 1971a; Sotelo and Angaut, 1973). In addition, large nuclear neurons have numerous spines and protrusions that eradiate from the somatic membrane. Presynaptic terminals are in direct apposition with somatic spines, protrusions, and with the smooth surface of the somatic plasma membrane making numerous synapses (Eager, 1968; O'Leary et al., 1972; Angaut and Sotelo, 1973; Chan-Palay, 1973a; Sotelo and Angaut, 1973). Interestingly, the soma of small nuclear neurons receives only sparse somatic afferent inputs (O'Leary et al., 1972; Angaut and Sotelo, 1973; Chan-Palay, 1973e). 
This disparity in somatic afferent inputs may reveal the underlying functional difference between large projection neurons and small interneurons of the AIN.

The location of synaptic inputs on the postsynaptic neuron and the distance from the site of action potential initiation can also determine their impact on signal output. Distal synapses can be subject to signal attenuation, whereas somatic synapses can directly influence the activity of the postsynaptic neuron (Rall, 1967; Williams and Stuart, 2003). Because of the proximity to the site of initiation, somatic synapses have the capacity to generate or shunt action potentials. Thus, changes on the somatic afferent inputs of large projection neurons have the potential of influencing behavior directly. In addition, the structural features of synapses have been correlated with function. For example, the size of the postsynaptic density (PSD) is highly correlated with the size of the presynaptic active zone. In addition, the size of the active zone is also correlated with the number of docked synaptic vesicles (Schikorski and Stevens, 1997, 2001), and with the probability of vesicle release (Burrone and Murthy, 2001; Li and Murthy, 2001; Branco et al., 2010; Marra et al., 2012). Moreover, the size of the PSD is proportional to the number of its receptors (Kharazia et al., 1996; Kharazia and Weinberg, 1999; Masugi-Tokita et al., 2007; Fukazawa et al., 2008; Tarusawa et al., 2009) which, in turn, determines the amplitude of the postsynaptic potential (Nusser et al., 1997). Therefore, the size and number of somatic PSDs on eyeblink projection neurons can be a meaningful indicator of functional changes (Kubota and Kawaguchi, 2000; Arellano et al., 2007b).

Eyeblink projection neurons are the final output from the cerebellum and can elicit eyeblink responses by activation of contralateral $\mathrm{RN}$ neurons which in turn activate eyeblink motoneurons. For this reason, behavioral changes associated with learning may be mediated by plasticity of somatic synapses. In this study, the role of somatic synapses on eyeblink projection neurons of the AIN as a function of EBC was investigated. 


\section{Material and Methods}

\section{Animals}

Eight adult male New Zealand rabbits (Oryctolagus cuniculus), about 2.5 months of age, and weighing approximately $2.5 \mathrm{~kg}$ at the beginning of the experiment were supplied by Harlan. Rabbits were divided into three groups, one experimental and two control groups. Rabbits were housed in individual cages, given free access to food and water, and maintained on a 12-hr lightdark cycle, all in accordance with the National Institute of Health guidelines. All procedures were approved by the West Virginia University Animal Care and Use Committee.

\section{Procedure}

All rabbits received a 60 minutes adaptation session a day prior to behavioral procedures. The paired (PD) group received 6 paired daily delay EBC training sessions. Each training session consisted of 60 presentations of a $400 \mathrm{~ms}$ tone-CS $(1 \mathrm{KHz}, 82 \mathrm{~dB})$ that was paired with a $100 \mathrm{~ms}$ airpuff-US (4 psi). The onset of the tone-CS preceded the onset of the airpuff-US by $300 \mathrm{~ms}$, then the two stimuli overlapped for $100 \mathrm{~ms}$ and co-terminated. Paired trial presentations were delivered $\sim 60$ seconds apart. The unpaired (UP) group received the same number of tone-CS and airpuff-US presentations as the PD group but in an explicitly unpaired manner. Each training session consisted of 120 unpaired trials of either a 400ms tone-CS or a 100ms airpuffUS delivered $\sim 30$ seconds apart. The control (CT) group was exposed to the same handling and apparatus set up procedures as the PD and UP groups but during each daily session they sat in the training chamber without receiving any stimuli. Eyeblink responses were measured as movement of the NM. The NM was chosen to measure eyeblinks responses instead of $O O M$ activity to conserve the integrity of the eyelid and motor endplates in the eyelid muscle for subsequent transneuronal tracing of the eyeblink premotor pathway. A CR was defined as any extension of the NM exceeding $0.5 \mathrm{~mm}$ that was initiated after the tone onset but prior to the airpuff onset.

\section{Apparatus}

The apparatus and recording procedures used in this study were developed by Gormezano (Gormezano et al., 1962), and have been previously described (Schreurs et al., 2000; Schreurs 
and Alkon, 2001). In brief, each rabbit was restrained in a Plexiglas box and placed in a soundattenuating, ventilated chamber (Coulbourn Instruments, Allentown, PA; Model E10-20). A stimulus panel containing a speaker and a $10-\mathrm{W}$ house light was mounted at a $45^{\circ}$ angle, $15 \mathrm{~cm}$ anterior to, and $15 \mathrm{~cm}$ above the rabbit's head. Ambient noise $(65 \mathrm{~dB})$ was provided by an exhaust fan. Air puffs to the cornea of the right eye were delivered by a programmable pressure regulator (ER3000, Tescom, Elk River, Minnesota, USA) connected to a (1 mm internal diameter) tube positioned $5 \mathrm{~mm}$ from the center of the cornea. A $1 \mathrm{~mm}$ hook connected to an Lshaped lever containing a freely moving ball and socket joint was attached to a 6-0 nylon loop sutured into, but not through, the NM. The other end of the lever was attached to a potentiometer (Novotechnik US Inc., Southborough, MA; Model P2201) that, in turn, was connected to a 12-bit analog-to-digital converter ( $5 \mathrm{~ms}$ sampling rate; $0.05 \mathrm{~mm}$ resolution). Individual analog-to-digital outputs were stored on a trial-by-trial basis for subsequent analysis. The LabVIEW system (National Instruments, Austin, TX) was used for stimuli delivery, data collection, and data analysis.

\section{PRV Injections}

The injection protocol of PRV was the same as that described in Experiment 1. Following conditioning, twelve microliters of PRV $\left(2.3 \times 10^{8}\right.$ plaque-forming units per milliliter $)$ divided into 4 injections of $3 \mu \mathrm{l}$ each were injected into the right upper eyelid. Each injection was transverse to the muscle fibers and delivered at a constant rate through the orbital, preseptal, and pretarsal portions of the $O O M$ to ensure adequate viral distribution. After injection, the syringe was left in place for 10 minutes to achieve complete viral absorption and to prevent any leakage.

\section{Western Immunoblotting}

The specificity of PSD95, and gephyrin primary antibodies in rabbit tissue was tested by western immunoblotting. In brief, rabbit cerebellar tissue was treated with protein inhibitors and proteases, homogenized, sonicated, and boiled for $5 \mathrm{~min}$. After centrifugation, the supernate was collected and $5 \mu \mathrm{l}$ was loaded on the gel along with a standard molecular weight marker (Seeblue, Invitrogen). After transferring, the nitrocellulose membrane $(0.45 \mu \mathrm{m}$, Invitrogen $)$ was blocked with 5\% non-fat milk and incubated in one of the primary antibodies for $4 \mathrm{hrs}$ at room temperature. The primary antibodies used in this study were a mouse monoclonal anti-PSD95 
(1:3,000 MA1-046 -clone: 7E3-1B8, Thermo Scientific), and a goat polyclonal anti-gephyrin (1:2,000, sc-6411-clone R-20, Santa Cruz Biotechnology Inc). Blots were washed and incubated with the corresponding fluorescent secondary antibody for $2 \mathrm{hrs}$ (a donkey anti-mouse Alexa 647, 1:1,000; and a donkey anti-goat Alexa 546, 1:1,000; Invitrogen, Carlsbad, CA). Images of western blot membranes were acquired using a fluorescent scanner (FujiFilm Fla-9000). In addition, the same combination of primary and secondary antibodies was used in subsequent immunofluorescent experiments.

\section{Tissue Processing}

Animals were euthanized with $1.0 \mathrm{cc}$ Euthasol (sodium pentobarbital, $390 \mathrm{mg} / \mathrm{ml}$ ) at 41/2 days after PRV-injections and perfused transcardially with $1.5 \mathrm{~L}$ of $0.9 \%$ saline $(\mathrm{pH} 7.4$ at room temperature) followed by $1 \mathrm{~L}$ of $4 \%$ formaldehyde. Brains were collected and placed in fixative for $4 \mathrm{hrs}$ and transferred to $30 \%$ sucrose for cryoprotection until they sank. Coronal $50-\mu \mathrm{m}$ sections were cut on a freezing microtome. All sections were marked on the contralateral side of injection to distinguish laterality.

\section{Immunofluorescence Labeling}

Cerebellar $50-\mu \mathrm{m}$ free-floating sections were incubated in citric acid $\left(\mathrm{pH} 6.0\right.$ at $60^{\circ} \mathrm{C}$ ) for $40 \mathrm{~min}$ to break protein crosslinks after formaldehyde fixation and to enhance antigen binding to its epitope. Sections were then washed in 0.1M PBS-tween, $\mathrm{pH} 7.4$ for $1 \mathrm{hr}$ and blocked with 5\% normal rabbit and donkey serum for $4 \mathrm{hrs}$ at room temperature. Next, sections were incubated with the first set of primary antibodies, a mouse monoclonal anti-PSD95, and a goat polyclonal anti-gephyrin overnight at $4^{\circ} \mathrm{C}$ in a $0.1 \mathrm{M}$ PBS-tween incubating solution ( 1:1,000; MA1-046, clone: 7E3-1B8, Thermo Scientific; and sc-6411, clone: R-20, Santa Cruz Biotechnology Inc., respectively). After several washes sections were incubated with their corresponding secondary antibodies for $6 \mathrm{hrs}$ at room temperature in an incubating solution (a donkey anti-mouse Alexa 647, 1:500; and a donkey anti-goat Alexa 546, 1:500; Invitrogen, Carlsbad, CA). After several washes sections were incubated with a third primary antibody, a rabbit polyclonal anti-L7 overnight at $4^{\circ} \mathrm{C}(1: 1,000$, the anti-L7 antibody was generously provided by Dr. Berrebi and has been described in detail previously (Oberdick, J., 1988; Berrebi et al., 1991a; Berrebi and Mugnaini, 1992). After several washes, sections were incubated with a secondary antibody for 6 
hrs at room temperature (1:500 donkey anti-rabbit Alexa 405, Invitrogen, Carlsbad, CA). Sections were then washed and mounted in gelatin-covered mounting slides and cover slipped with Fluoromount-G (SouthernBiotech).

\section{Image acquisition}

Rabbit cerebellar sections were visualized using a confocal laser microscope (Zeiss LSM 710; Carl Zeiss International). Images of PRV-labeled neurons were acquired using 633, 543, 488, and $405 \mathrm{~nm}$ lasers. Confocal microscope settings were set to obtain images with optimum resolution $(\operatorname{Res}=0.51 \mathrm{x} \lambda / \mathrm{NA})$. Images were acquired using sequential multichannel frame scan, averaged twice (Nyquist sampling frequency of 2), with a pinhole aperture 1AU, scanner zoom of 1.4X; and 1024 x1024 pixel resolution. The filters were set manually to detect the spectral peak of each fluorophore and to avoid emission cross-talk. First, PRV-labeled neurons in the AIN were visualized using a 10X objective (NA 0.16). Next, three images through each PRVlabeled neuron were collected at different z-coordinates using a $63 \mathrm{X}$ oil-immersion objective (NA 1.4). These images were taken: (1) at the center of the neuron where the nucleus appeared the largest; (2) $\sim 2 \mu \mathrm{m}$ below the center; and (3) $\sim 2 \mu \mathrm{m}$ above the center of the PRV-labeled neuron. The distance between images ensured that each PSD was counted only once. In addition, only PRV-labeled neurons with a visible nucleus were included in this study to avoid neuron over counting. Images were exported as tiff files for data analysis.

\section{Image analysis}

Fluorescently labeled presynaptic and postsynaptic densities (PSD) were measured using the "Analyze Particles" module of ImageJ (National Institute of Health, USA) which has been used previously to measure the number and size of PSDs (Arellano et al., 2007a; Ito and Takeichi, 2009; Burli et al., 2010; Garcia-Junco-Clemente et al., 2010; Schmitz et al., 2011; Andlauer and Sigrist, 2012; Chemin et al., 2012). Tiff images were renamed and assigned a random number using "Bulk Rename Utility" software to remove any information from the subject and experimental group. All measurements were obtained blind to the experimental conditions. Merged tiff images were opened as RGB images and the contour of the soma membrane of each AIN PRV-labeled neuron was delineated. A $0.9 \mu \mathrm{m}$-wide band around the soma of each neuron that included presynaptic and postsynaptic densities was generated by ImageJ (make a band 
tool). Information contained within the band was selected and separated into three different 8-bit grey images (one for each RGB channel). The red channel contained information from gephyrin-immunolabeled densities, the green channel contained information from PSD95immunolabeled densities, and the blue channel contained information from L7-immunolabeled densities. Images were thresholded and the area, perimeter, and Feret's diameter of each PSD puncta was measured using the "Analyze Particle" module of ImageJ and exported to Excel for further analysis.

\section{Synapse estimations}

To estimate the number of excitatory and inhibitory somatic synapses on eyeblink premotor neurons of the AIN, we used a designed-based approach. It is important to note here that the focus of this experiment was to compare the effect of different behavioral procedures on the number of somatic synapses on a distinct and functionally homogeneous group of AIN neurons, eyeblink projection neurons. For this reason, stereological approaches commonly used to estimate the number of synapses within a brain volume or region that is comprised of multiple neuronal populations was not suited for this study. Instead, each eyeblink-labeled neuron was equally sampled and measurements were obtained systematically. Stereological techniques provide meaningful estimates of the geometry of 3D structures from measurements obtained on 2D images (Weibel 1979; Cruz-Orive 1997; DeHoff 2000). Using these techniques it is possible to reconstruct the size and distribution of synapses and to relate their size and number to an appropriate reference, such as the length or surface area of the somatic membrane of eyeblink projection neurons. Stereological principles and equations used here were derived from those described previously (Mayhew, 1979; Calhoun et al., 1996; West, 1999). The number (N) of PSDs in a given length $\left(\mathrm{N}_{\mathrm{L}}\right)$ was calculated by dividing the number of PSDs by the length (L) of the somatic membrane:

$$
\mathrm{N}_{\mathrm{L}}=\mathrm{N} / \mathrm{L}
$$

The number of PSDs in a given area $\left(\mathrm{N}_{\mathrm{A}}\right)$ depends not only on the number of PSDs in a length, $\mathrm{N}_{\mathrm{L}}$, but also on the size of the PSDs (Mayhew, 1979; West, 1999; Boyce et al., 2010). The size of PSDs was determined based on the assumption that PSDs are flat circular disc-like structures located on the surface membrane of the postsynaptic neuron. In a cross-sectional section, a 
PSDs appears as a line. The length of each line (d) was measured using the Feret's diameter of each PSD puncta (see Image Analysis). The length, $d$ is never larger than the diameter (D) of the PSD and has an $87 \%$ probability of having a length that falls between $\mathrm{D}$ and $1 / 2 \mathrm{D}$, whereas only a $13 \%$ probability of having a length that is less than $1 / 2 \mathrm{D}$. The mean length, $\overline{\mathrm{d}}$ corresponds to $\pi / 4$ of the true PSD diameter, D (Mayhew, 1979). Using the counterpart of this equation, the diameter of the different PSDs can be calculated by multiplying the mean PSD length, $\overline{\mathrm{d}}$ by $4 / \pi$ to obtain the estimated PSD mean diameter, $(\overline{\mathrm{D}})$ :

$$
\overline{\mathrm{D}}_{\mathrm{PSD}}=(4 / \pi) \cdot \overline{\mathrm{d}}_{\mathrm{PSD}}
$$

The diameter of presynaptic and postsynaptic densities was calculated for each eyeblink AIN neuron. Next, the number of PSDs in a given unit area $\left(\mathrm{N}_{\mathrm{A}}\right)$ was estimated by multiplying the $\mathrm{N}_{\mathrm{L}}$ by a unit area (A) and dividing it by $\overline{\mathrm{D}}$ :

$$
\mathrm{N}_{\mathrm{A}}=\left(\mathrm{N}_{\mathrm{L}} \cdot \mathrm{A}\right) / \overline{\mathrm{D}}
$$

Finally, the estimated number of PSDs per neuron $\left(\mathrm{N}_{\mathrm{S}}\right)$ was calculated by multiplying the number of PSDs per unit area, $\mathrm{N}_{\mathrm{A}}$ by the somatic surface area $(\mathrm{S})$ of the neuron:

$$
\mathrm{N}_{\mathrm{S}}=\mathrm{N}_{\mathrm{A}} \cdot \mathrm{S}
$$

It is important to note here, that a possible source of bias was introduced by the use of coronal sections. The use of sections with multiple orientations is desired for unbiased estimates in 3D structures. However, using multiple orientation planes would have introduced additional variables and any differences across groups would not longer be attributed exclusively to the experimental condition. For this reason, a single orientation plane was used. In addition, this possible source of bias is systematic and would affect all groups equally.

\section{Statistical analyses}

Behavioral data were analyzed using repeated measures Analysis of Variance (ANOVA) with the significance level set at $p<.05$ (SPSS 14.0, Chicago, Illinois). Presynaptic and postsynaptic densities data were analyzed using linear mixed effects models generated with SAS/STAT software with the significance level set at $p<.05$ (Version 9.3, SAS Institute Inc., Cary, NC) and 
with the assistance from the Statistics Department at WVU. These models used a random effect to control for the variability within a neuron and within a subject as multiple measurements were made on each, and provided more accurate estimates of the effect of the experimental or independent variable on the outcome of the dependent variables (PSD95, gephyrin, L7, and somatic area). Separate models were constructed for each outcome while controlling for the others.

\section{Results}

\section{Eyeblink conditioning}

In this study, the effect of EBC in the number and size of excitatory and inhibitory somatic synapses of eyeblink premotor neurons of the AIN was investigated. Rabbits were divided into 3 groups that underwent different behavioral procedures. The PD group $(n=3)$ received paired delay stimuli presentations of a $400 \mathrm{~ms}$ tone-CS followed by a $100 \mathrm{~ms}$ airpuff-US. These two stimuli briefly overlapped and co-terminated. The UP group $(n=3)$ was a control group and received the same number of stimuli as the PD group but in an explicitly unpaired manner (the stimuli were presented separately and they were never paired together). The CT group $(n=2)$ was an exposure control group and received the same routine and handling as the other two groups but sat in the training chamber without receiving any stimuli (neither tones nor airpuffs).

Figure 20 shows traces of individual eyeblink responses of three representative subjects from the PD, UP, and CT group, respectively. Eyeblink responses were measured by the movement of the $\mathrm{NM}$ and are shown as traces of every $10^{\text {th }}$ trial for each of the 6 days of training. The development of learning can be seen in a representative subject from the PD group as eyeblink CRs gradually developed during EBC (Figure 20 A). Initially, during paired tone-CS and airpuff-US presentations, the subject does not blink to the sound of the tone, whereas the airpuffUS consistently elicits an eyeblink UR (evident by the $10^{\text {th }}-40^{\text {th }}$ UR trace on day 1 ). After repeated paired stimulus presentations, the subject begins to blink to the tone-CS in anticipation to the airpuff-US. The emergence of the first eyeblink CRs can be seen on the $50^{\text {th }}$ paired trial on day 1 (Figure $20 \mathrm{~A}$ ). Notably, the peak of the CR gradually shifts during training to peak 
about the time when the airpuff-US occurs. On the other hand, explicitly unpaired presentations of the tone-CS and airpuff-US fail to produce CRs, shown by eyeblink traces of a representative subject from the UP group (Figure 20 B). In this case, the tone-CS does not elicit an eyeblink by itself and because it was never paired with the airpuff-US, an association was never formed. Traces of a representative subject of the CT group are shown in Figure 20 C. Note that subjects in the CT group did not receive any stimuli, however, spontaneous eyeblink responses were monitored during the interval over which CRs were measured in the paired group.

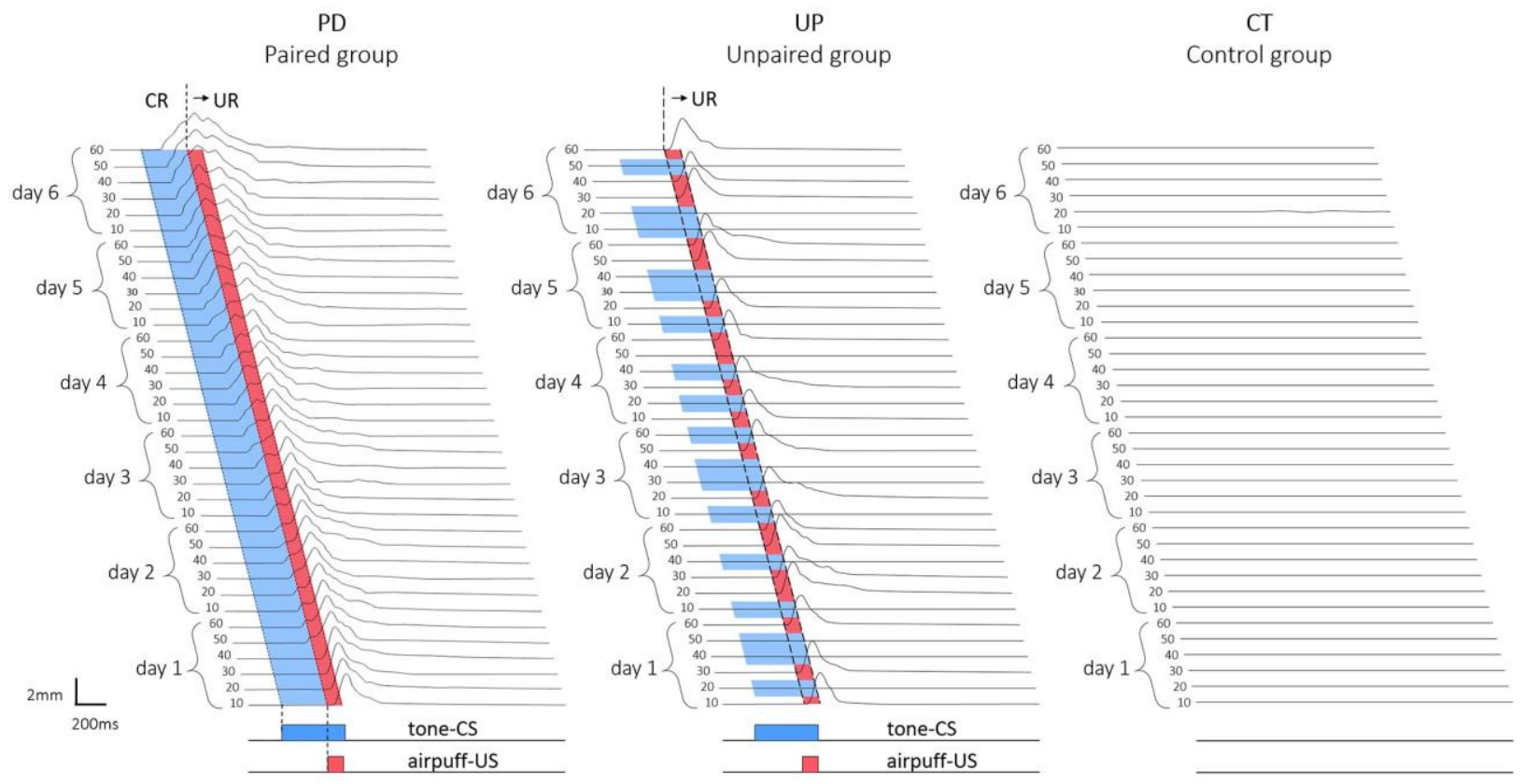

Figure 20. Development of conditioned responses during eyeblink conditioning.

Eyeblink responses were measured as movement of the nictitating membrane (NM) in the paired PD, unpaired UP and control CT groups during 6 days of behavioral procedures.

Figure 21 shows the percentage CRs for the PD, UP, and CT groups across the 6 days of training. The PD group acquired CRs rapidly showing over $70 \% \mathrm{CRs}$ on the second day and over $90 \%$ CRs on the third day of paired delay training. On the fifth day of training, the PD group reached asymptotic levels over $95 \% \mathrm{CRs}$ and these levels were maintained on the following sixth day of training. In contrast, the UP and CT subjects showed only marginal levels of responding of no more than $3 \%$ on any given day. Analysis of variance (ANOVA) confirmed 
these observations with significant effects of groups $[F(2,19)=1726.51, p<.0001]$, days $[F(5,20)=46.39, p<.0001]$, and groups $\mathrm{x}$ days $[F(10,20)=51.55, p<.0001]$.

\section{Eyeblink Conditioning}

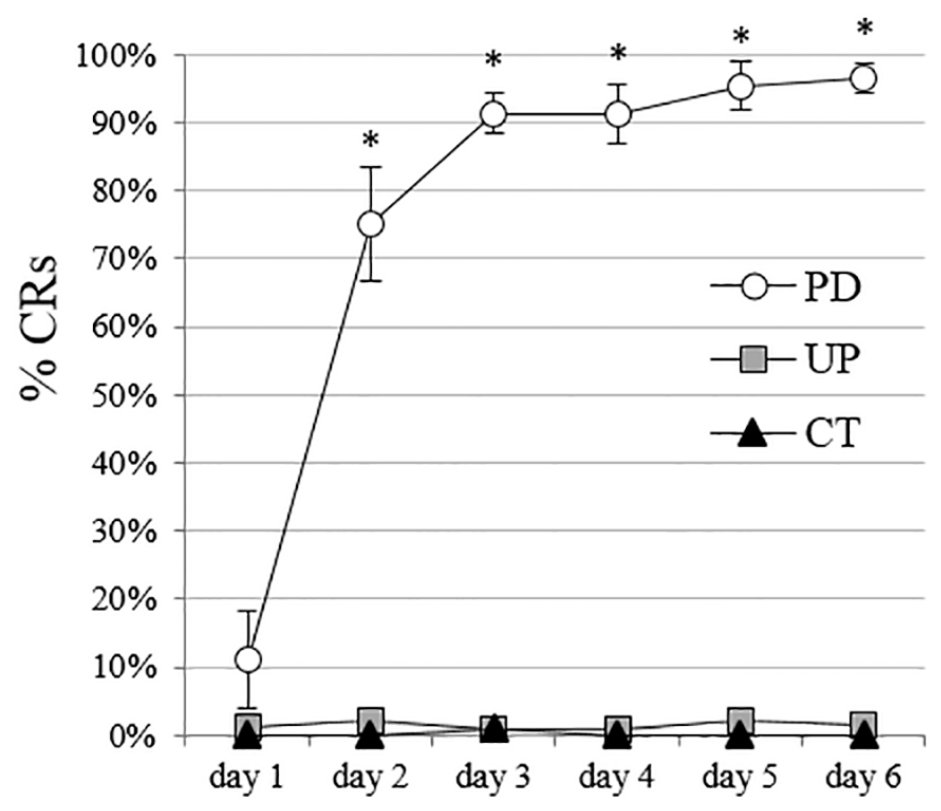

Figure 21. Mean percentage conditioned responses (CR).

The mean ( \pm SEM) \% CRs during six daily sessions in the paired (PD), unpaired (UP), and control (CT) groups. The PD group showed a significant increase in the \%CRs as a function of days and days $\mathrm{x}$ group $(* \mathrm{P}<0.001)$.

\section{Eyeblink projection neurons in the AIN}

In order to study learning-specific synaptic changes as a function of $\mathrm{EBC}$, the number of excitatory and inhibitory somatic synapses was measured on all eyeblink AIN neurons labeled with PRV. Figure 22 shows the experimental procedures. Figure 22 A shows a diagram of the pathways involved in EBC. Information about the tone-CS is conveyed to the AIN by MF collaterals and to PCs by way of GCs. Information about the airpuff-US is conveyed to the AIN by CF collaterals and to PCs. There are 3 basic types of AIN neurons. The first type is 
comprised of large glutamatergic neurons that project to the contralateral RN, shown in green. The second type is comprised of small GABAergic neurons that project to the contralateral IO and form the nucleo-olivary inhibitory feedback loop, shown in dark gray. The third type is comprised of small GABAergic and/or glycinergic interneurons that project locally, shown in purple. Glutamatergic projection neurons integrate excitatory inputs from MFs and CFs, and inhibitory inputs from PCs and local interneurons to form the final output from the cerebellum. In this manner, eyeblink projection neurons are the ultimate output from the cerebellum and responsible for the execution of the eyeblink CR.

After behavioral procedures, PRV was injected into the $O O M$ of the trained eye to label eyeblink projection neurons of the AIN neurons that are synaptically connected and part of the eyeblink premotor pathway. Figure $22 \mathrm{~B}$ shows the retrograde transsynaptic spread of PRV through the eyeblink premotor pathway. After injections, PRV is up taken by first-order $\left(1^{\circ}\right)$ motoneurons in the $7 \mathrm{~N}$ innervating the OOM. Next, PRV spreads to synaptically connected second-order $\left(2^{\circ}\right)$ premotor neurons in the contralateral RN. Finally, PRV spreads to synaptically connected thirdorder $\left(3^{\circ}\right)$ premotor neurons in the ipsilateral AIN (eyeblink projection neurons). The time period necessary for PRV to label $3^{\circ}$ glutamatergic projection neurons in the AIN was assessed in Chapter 3 and corresponded to 4 1/2 days after injections (Gonzalez-Joekes and Schreurs, 2012). Four and a half days after PRV injections, brains were collected, sectioned, and immunolabeled with specific synaptic markers. The location within the AIN where $3^{\circ}$ eyeblink projection neurons labeled with PRV were found is shown in Figure 22 C. Eyeblink projection neurons of the AIN were found in the dorsolateral AIN, ipsilateral to the trained eye, and consistent with the location of eyeblink AIN found in other studies (McCormick and Thompson, 1984a; Berthier and Moore, 1990; Morcuende et al., 2002; Gonzalez-Joekes and Schreurs, 2012). Figure 22 D shows a group of 3 PRV-labeled neurons found in the dorsolateral AIN of a rabbit in the in the CT group. These neurons were large and consistent with the size of glutamate-ir neurons found in Chapter 3 (Gonzalez-Joekes and Schreurs, 2012). 
A
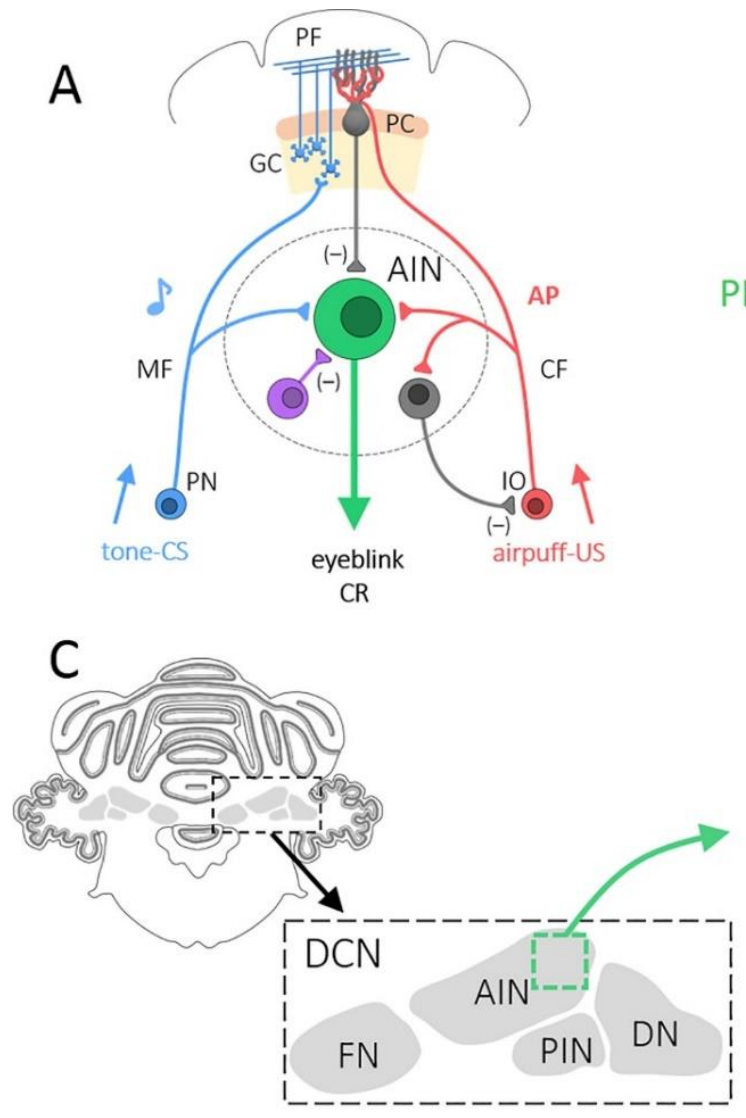

$E$

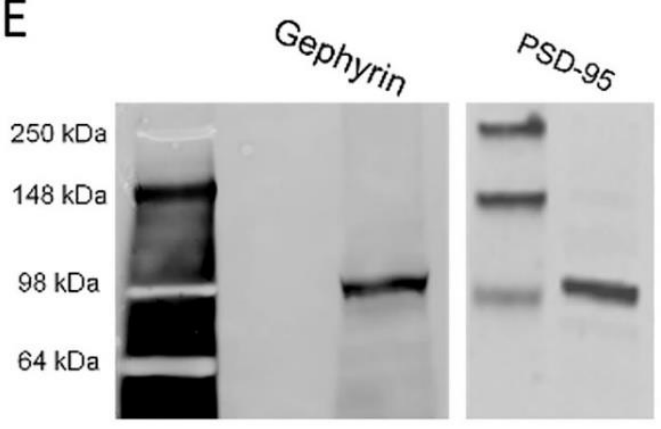

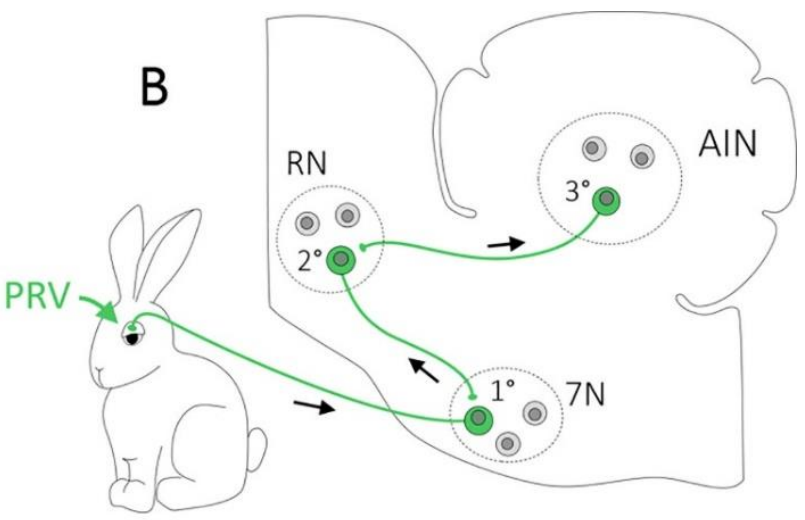

D

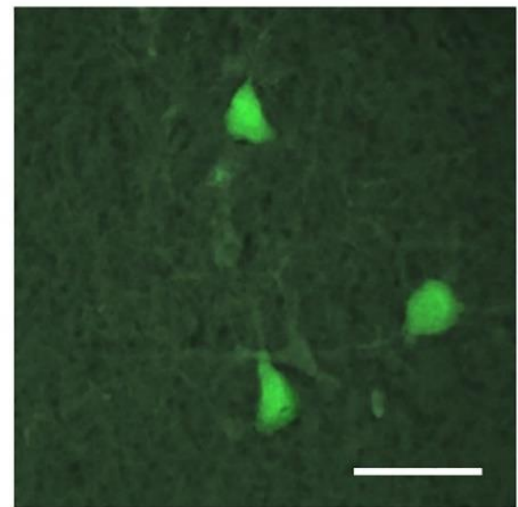

$\mathrm{F}$

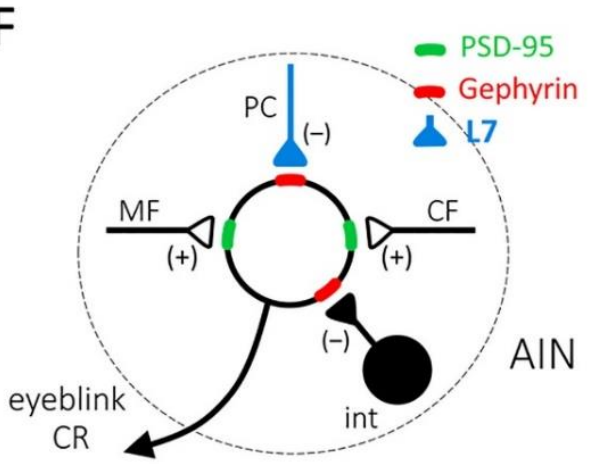

Figure 22. Somatic synapses on identified eyeblink projection neurons of the AIN.

$\mathbf{A}$, afferent inputs on AIN neurons form mossy fibers (MF), climbing fibers (CF), Purkinje cells (PC), and interneurons (purple). B, retrograde transsynaptic tracing of the eyeblink projection neurons of the AIN. C, coronal section showing the location of eyeblink projection neurons in the anterior interpositus nucleus (AIN) of the deep cerebellar nuclei (DCN). D, three PRV-labeled eyeblink projection neurons of the AIN. E, specificity of PSD95 and gephyrin antibodies in rabbit cerebellar tissue. F, schematic of excitatory and inhibitory synapses on AIN neurons. Excitatory somatic synapses from MFs and CFs are labeled with PSD95. Inhibitory synapses from PC and interneurons and labeled with gephyrin. PC terminals are labeled with L7. 
Specific markers were used to identify excitatory and inhibitory synapses. Synapses are a functional unit, comprised of two specialized structures in direct apposition, the presynaptic active zone and the postsynaptic density. PSD95 was used to identify excitatory synapses. PSD95 is a specific marker that labels the postsynaptic density of excitatory synapses. Gephyrin was used to identify inhibitory synapses. Gephyrin is a specific marker that labels the postsynaptic density of inhibitory GABAergic and glycinergic synapses (Fritschy et al., 2008). The specificity of the primary antisera against PSD95 and gephyrin was confirmed by western blot analysis on rabbit brain tissue, shown in Figure 22 E. Because a large proportion of inhibitory synapses originate from PCs, a PC specific marker, L7 was used to identify PC presynaptic terminals. Postsynaptic densities labeled with gephyrin were classified as inhibitory synapses and further divided into two groups. Inhibitory synapses that were in direct apposition with a PC presynaptic terminal (labeled with L7) were classified as PC synapses. Inhibitory synapses that were not in direct apposition with a PC terminal were classified as interneuron synapses. Figure 22 F shows a schematic diagram of the excitatory and inhibitory afferent inputs in the AIN. Excitatory synapses from MFs and CFs were labeled with PSD95, shown in

green. Inhibitory synapses from PCs and interneurons were labeled with gephyrin, shown in red. PC terminals were labeled with L7, shown in blue.

A total of 59 eyeblink AIN neurons were found in the PD group $(n=3), 76$ eyeblink AIN neurons were found in the UP group $(\mathrm{n}=3)$, and 17 eyeblink AIN neurons were found in the CT group (from a single subject). The average number $( \pm S E M)$ of eyeblink AIN neurons per subject was $21.7 \pm 2.5$ which is consistent with the average of 20 glutamate-ir neurons found in Chapter 3 at 4 1/2 days after PRV injections.

\section{Presynaptic and postsynaptic densities}

Presynaptic PC terminals, and postsynaptic excitatory and inhibitory densities on an eyeblink projection neuron of the AIN are shown in Figure 23. Figure $23 \mathrm{~A}$ shows a PRV-labeled 
eyeblink premotor neuron in the AIN. Excitatory postsynaptic densities labeled with PSD95 are shown in Figure 23 B. Purkinje cell terminals labeled with L7 are shown in Figure 23 C. inhibitory PSDs labeled with gephyrin are shown in Figure 23 D. Figure 23 E shows colocalization of excitatory (PSD95) and inhibitory (gephyrin) postsynaptic densities. Figure $23 \mathbf{~ F}$ shows co-localization of inhibitory (gephyrin) postsynaptic densities and PC terminals. A merged image showing the co-localization of excitatory (PSD95) and inhibitory (gephyrin) postsynaptic densities, and PC terminals (L7) is shown in Figure 23 G. A $0.9 \mu$ m-wide band drawn around the somatic membrane of the PRV-labeled eyeblink neuron that contains presynaptic and postsynaptic densities is shown in Figure $23 \mathbf{~ H}$. Information within the band was selected and analyzed separately for PSD95, gephyrin, and L7 using the "Analyze Particle" module of ImageJ (see Methods section for more details). Figure 23 I is a merged image showing excitatory synapses in green, inhibitory synapses in red, and Purkinje cell terminals in blue. The number, and size of somatic excitatory and inhibitory postsynaptic densities, and PC terminals were analyzed on three optical sections for each eyeblink AIN neuron and averaged to obtain groups values.

\section{Number of somatic synapses}

The number of PSDs in a given length of somatic membrane was calculated by dividing the number of PSDs by the length of the somatic membrane to obtain the number of somatic synapses per $\mu \mathrm{m}$. Next, the size of each PSD was calculated to obtain the number of somatic synapses per unit area $\mu \mathrm{m}^{2}$ (see Methods section). Finally, the number of somatic synapses per neuron was estimated by multiplying the number of PSDs per $\mu \mathrm{m}^{2}$ by the somatic surface area of the neuron. The results obtained for the number of somatic synapses per $\mu \mathrm{m}$, per $\mu \mathrm{m}^{2}$, and per neuron are shown in Table 2. 

Table 2. Somatic synapses on eyeblink projection neurons of the AIN.

\begin{tabular}{|c|c|c|c|c|}
\hline & & $\begin{array}{c}\text { PD } \\
\text { Paired Group } \\
\end{array}$ & $\begin{array}{c}\text { UP } \\
\text { Unpaired Group }\end{array}$ & $\begin{array}{c}\text { CT } \\
\text { Control Group } \\
\end{array}$ \\
\hline Subjects & & 3 & 3 & 1 \\
\hline Eyeblink AIN neurons & & 59 & 76 & 17 \\
\hline Soma area $\left(\mu \mathrm{m}^{2}\right)$ & & $475 \pm 11^{* *}$ & $436 \pm 9 *$ & $395 \pm 16$ \\
\hline Number of synapses pe & $\mathrm{PC}$ terminal & $3.27 \pm 0.12$ & $3.15 \pm 0.10$ & $3.22 \pm 0.19$ \\
\hline $\begin{array}{l}\text { Mean Diameter } \overline{\mathrm{D}} \text {, of } \\
\text { somatic densities }(\mu \mathrm{m})\end{array}$ & $\begin{array}{r}\text { PSD95 } \\
\text { Gephyrin } \\
\text { L7 }\end{array}$ & $\begin{array}{l}0.413 \pm 0.019 \\
0.392 \pm 0.016 \\
1.202 \pm 0.048\end{array}$ & $\begin{array}{l}0.441 \pm 0.017 \\
0.406 \pm 0.015 \\
1.093 \pm 0.039\end{array}$ & $\begin{array}{l}0.429 \pm 0.031 \\
0.411 \pm 0.033 \\
1.153 \pm 0.070\end{array}$ \\
\hline $\begin{array}{l}\text { Number of somatic } \\
\text { densities / } \mu \mathrm{m}\end{array}$ & $\begin{array}{r}\text { PSD95 } \\
\text { Gephyrin } \\
\text { L7 } \\
\text { Total }\end{array}$ & $\begin{array}{l}0.105 \pm 0.004^{* *} \\
0.283 \pm 0.009 \\
0.213 \pm 0.007 \\
0.388 \pm 0.014\end{array}$ & $\begin{array}{l}0.086 \pm 0.003 \\
0.295 \pm 0.008 \\
0.194 \pm 0.006 \\
0.381 \pm 0.013\end{array}$ & $\begin{array}{l}0.092 \pm 0.006 \\
0.292 \pm 0.016 \\
0.203 \pm 0.013 \\
0.384 \pm 0.024\end{array}$ \\
\hline $\begin{array}{l}\text { Number of somatic } \\
\text { densities } / \mu \mathrm{m}^{2}\end{array}$ & $\begin{array}{r}\text { PSD95 } \\
\text { Gephyrin } \\
\text { L7 } \\
\text { Total }\end{array}$ & $\begin{array}{l}0.254 \pm 0.010^{* *} \\
0.721 \pm 0.024 \\
0.177 \pm 0.007 \\
0.975 \pm 0.035\end{array}$ & $\begin{array}{l}0.195 \pm 0.008 \\
0.726 \pm 0.022 \\
0.168 \pm 0.005 \\
0.921 \pm 0.030\end{array}$ & $\begin{array}{l}0.215 \pm 0.013 \\
0.711 \pm 0.041 \\
0.171 \pm 0.012 \\
0.925 \pm 0.055\end{array}$ \\
\hline $\begin{array}{l}\text { Number of somatic } \\
\text { densities per neuron }\end{array}$ & $\begin{array}{r}\text { PSD95 } \\
\text { Gephyrin } \\
\text { L7 } \\
\text { Total }\end{array}$ & $\begin{aligned} 483 & \pm 19^{* * *} \\
1371 & \pm 37^{* *} \\
337 & \pm 15^{*} \\
1854 & \pm 70\end{aligned}$ & $\begin{aligned} 337 & \pm 13 \\
1270 & \pm 34^{*} \\
294 & \pm 11 \\
1610 & \pm 59\end{aligned}$ & $\begin{aligned} 345 & \pm 22 \\
1123 & \pm 51 \\
270 & \pm 22 \\
1462 & \pm 106\end{aligned}$ \\
\hline $\begin{array}{l}\text { Number of somatic } \\
\text { synapses } / \mu \mathrm{m}\end{array}$ & $\begin{array}{r}\text { Excitatory } \\
\text { Inhibitory } \\
\text { Purkinje Cell } \\
\text { Interneuron } \\
\text { Total }\end{array}$ & $\begin{array}{l}0.105 \pm 0.004 * * * \\
0.283 \pm 0.009 \\
0.227 \pm 0.007 \\
0.055 \pm 0.003 \\
0.388 \pm 0.014\end{array}$ & $\begin{array}{l}0.086 \pm 0.003 \\
0.295 \pm 0.008 \\
0.215 \pm 0.006 \\
0.080 \pm 0.003 * * \\
0.381 \pm 0.013\end{array}$ & $\begin{array}{l}0.092 \pm 0.006 \\
0.292 \pm 0.016 \\
0.226 \pm 0.011 \\
0.066 \pm 0.005 \\
0.384 \pm 0.024\end{array}$ \\
\hline $\begin{array}{l}\text { Number of somatic } \\
\text { synapsess } / \mu \mathrm{m}^{2}\end{array}$ & $\begin{array}{r}\text { Excitatory } \\
\text { Inhibitory } \\
\text { Purkinje Cell } \\
\text { Interneuron } \\
\text { Total }\end{array}$ & $\begin{array}{l}0.254 \pm 0.010^{* * *} \\
0.721 \pm 0.024 \\
0.580 \pm 0.022 \\
0.141 \pm 0.006 \\
0.975 \pm 0.035\end{array}$ & $\begin{array}{l}0.195 \pm 0.008 \\
0.726 \pm 0.022 \\
0.530 \pm 0.018 \\
0.196 \pm 0.008^{* *} \\
0.921 \pm 0.030\end{array}$ & $\begin{array}{l}0.215 \pm 0.013 \\
0.711 \pm 0.041 \\
0.549 \pm 0.037 \\
0.162 \pm 0.010 \\
0.925 \pm 0.055\end{array}$ \\
\hline $\begin{array}{l}\text { Number of somatic } \\
\text { synapses per neuron }\end{array}$ & $\begin{array}{r}\text { Excitatory } \\
\text { Inhibitory } \\
\text { Purkinje Cell } \\
\text { Interneuron } \\
\text { Total }\end{array}$ & $\begin{aligned} 483 & \pm 19 * * * \\
1371 & \pm 37 * * \\
1102 & \pm 33 * * * \\
269 & \pm 15 \\
1854 & \pm 70^{* *}\end{aligned}$ & $\begin{aligned} 336 & \pm 13 \\
1270 & \pm 32 * \\
927 & \pm 28 \\
343 & \pm 17 * * \\
1610 & \pm 59\end{aligned}$ & $\begin{aligned} 345 & \pm 22 \\
1123 & \pm 49 \\
867 & \pm 40 \\
256 & \pm 22 \\
1462 & \pm 106\end{aligned}$ \\
\hline $\begin{array}{l}\text { Percentage of somatic } \\
\text { synapses }(\%)\end{array}$ & $\begin{array}{r}\text { Excitatory } \\
\text { Inhibitory } \\
\text { Purkinje Cell } \\
\text { Interneuron }\end{array}$ & $\begin{array}{l}26.07 \pm 1.04 \\
73.93 \pm 2.01 \\
59.42 \pm 1.79 \\
14.51 \pm 0.79\end{array}$ & $\begin{array}{l}20.89 \pm 0.82 \\
78.85 \pm 1.97 \\
57.56 \pm 1.73 \\
21.29 \pm 1.03\end{array}$ & $\begin{array}{l}23.61 \pm 1.58 \\
76.80 \pm 3.33 \\
59.33 \pm 2.76 \\
17.47 \pm 1.52\end{array}$ \\
\hline
\end{tabular}


The number of somatic synapses (excitatory and inhibitory) on each eyeblink AIN neuron was calculated and compared across groups. Figure 24 shows the average number of somatic synapses per neuron in the PD, UP, and CT group. The average number of somatic synapses was $1,854 \pm 70$ in the PD group, 1,610 \pm 59 in the UP group, and 1,462 \pm 106 in the CT group. The number of somatic synapses in the PD group was significantly larger than the number found in the UP $[F(1,133)=7.27, p<.008]$ and CT $[F(1,74)=7.82, p<.007]$ groups. This significant difference in the number of somatic synapses found in the PD group represents a $17 \%$ increase compared to the UP group, and a $27 \%$ increase compared to the CT group. The number of somatic synapses found in the UP group was $10 \%$ larger than the number found in the CT group, but this difference was not significant $[F(1,91)=1.22, p<.273]$.

\section{Total Somatic Synapses}

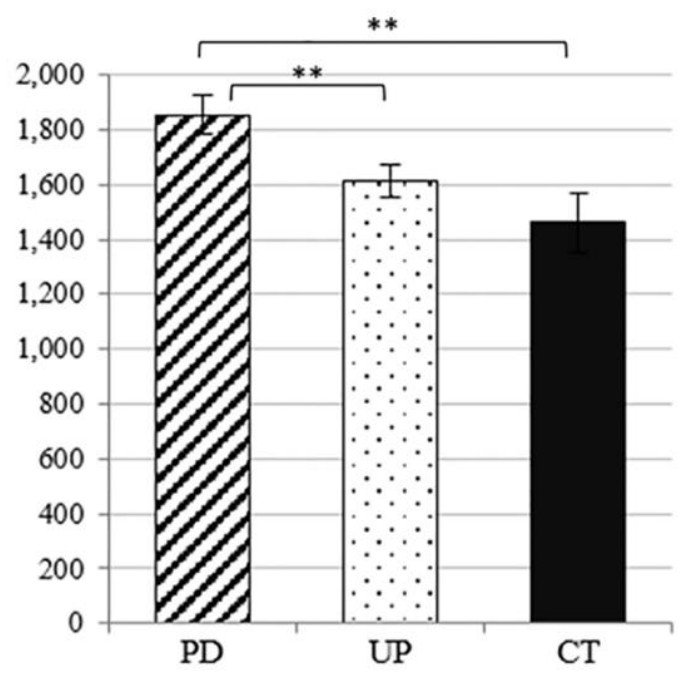

Figure 24. Average number of somatic synapses on eyeblink projection neurons of the AIN.

The mean $( \pm$ SEM) number of excitatory and inhibitory postsynaptic densities on the soma of eyeblink projection neurons of the AIN in the paired (PD), unpaired (UP), and control (CT) groups. The number of somatic synapses in the PD group was significantly larger than those in the UP and CT groups $(* * \mathrm{P}<0.01)$. 


\section{Excitatory somatic synapses}

Figure 25 shows the average number of excitatory PSDs per neuron in the PD, UP, and CT groups. The number of excitatory somatic synapses was $483 \pm 19$ in the PD group, $336 \pm 13$ in the UP group, and $345 \pm 22$ in the CT group. The number of excitatory synapses in the PD group was significantly larger than both the UP $[F(1,133)=42.52, p<.0001]$ and CT $[F(1,74)=$ $13.48, p<.0001]$ group. This significant difference in the number of excitatory synapses found in the PD group represents a $42 \%$ increase compared to both the UP and CT group. On the other hand, excitatory synapses on the UP and CT group were similar and not significantly different from each other.

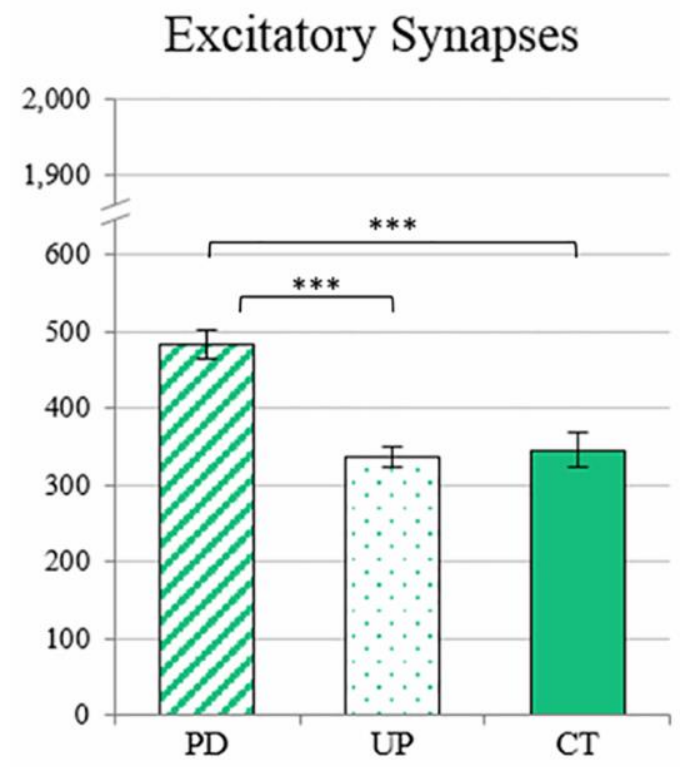

Figure 25. Average number of excitatory somatic synapses on eyeblink projection neurons of the AIN.

The mean $( \pm$ SEM) number of excitatory postsynaptic densities on the soma of eyeblink projection neurons of the AIN in the paired (PD), unpaired (UP), and control (CT) groups. The number of excitatory somatic synapses in the PD group was significantly larger those in the UP and CT groups ( $* * * \mathrm{P}<0.001)$. 


\section{Inhibitory somatic synapses}

Figure 26 shows the average number of inhibitory PSDs per neuron in the PD, UP, and CT groups. The number of inhibitory somatic synapses was 1,371 \pm 37 in the PD group, 1,270 \pm 32 in the UP group, and 1,123 \pm 49 in the CT group. The number of inhibitory synapses in the PD group was significantly larger than both the UP $[F(1,133)=4.35, p<.039]$ and CT $[F(1,74)=$ $11.36, p<.001]$ group. In addition, The number of inhibitory PSDs in the UP group was significantly larger than the CT group $[F(1,91)=4.34, p<.040]$. This significant difference in the number of inhibitory synapses found in the PD group represents a $10 \%$ increase compared to the UP group, and a $22 \%$ increase compared to the CT group. The number of inhibitory synapses in the UP group represents a $12 \%$ increase compared to the CT group and it was also significant.

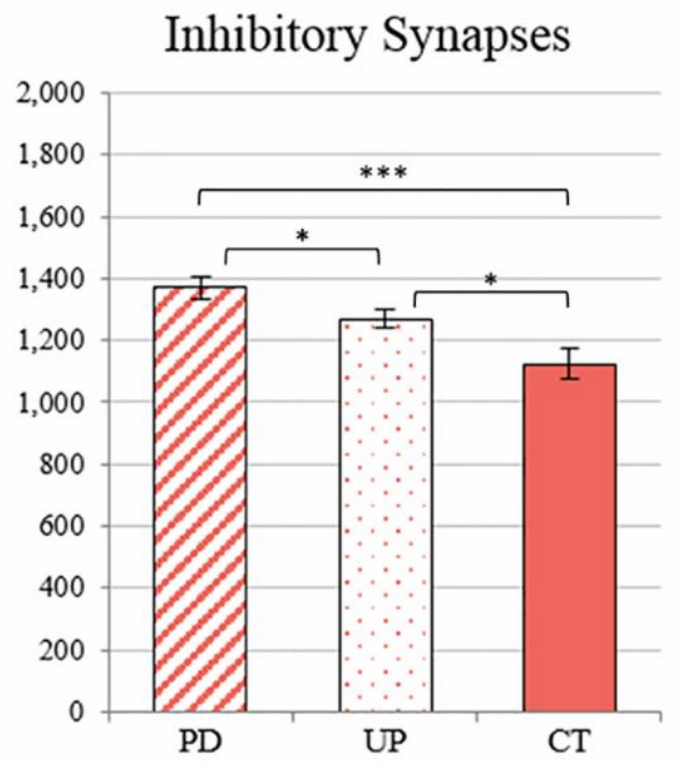

Figure 26. Number of inhibitory somatic synapses on eyeblink projection neurons of the AIN.

The mean $( \pm$ SEM) number of inhibitory postsynaptic densities on the soma of eyeblink projection neurons of the AIN in the paired (PD), unpaired (UP), and control (CT) groups. The number of inhibitory somatic synapses in the PD group was significantly larger those in the UP and CT groups, whereas the UP group was significantly larger than the CT group $(* \mathrm{P}<0.05$, *** $\mathrm{P}<0.001)$. 


\section{Purkinje cell terminals}

Figure 27 shows the average number of PC terminals per neuron in the PD, UP, and CT groups. The number of PC terminals was $337 \pm 15$ in the PD group, $294 \pm 11$ in the UP group, and $270 \pm$ 22 in the CT group. The number of PC terminals in the PD group was significantly larger than the UP $[F(1,133)=5.49, p<.021]$ and CT $[F(1,74)=4.95, p<.030]$ group. This significant difference in the number of PC terminals in the PD group represents a $14 \%$ increase compared to the UP group and a $25 \%$ increase compared to the CT group. The number of PC terminals in the UP group was $10 \%$ larger than the number found in the CT group but this difference was not significant.

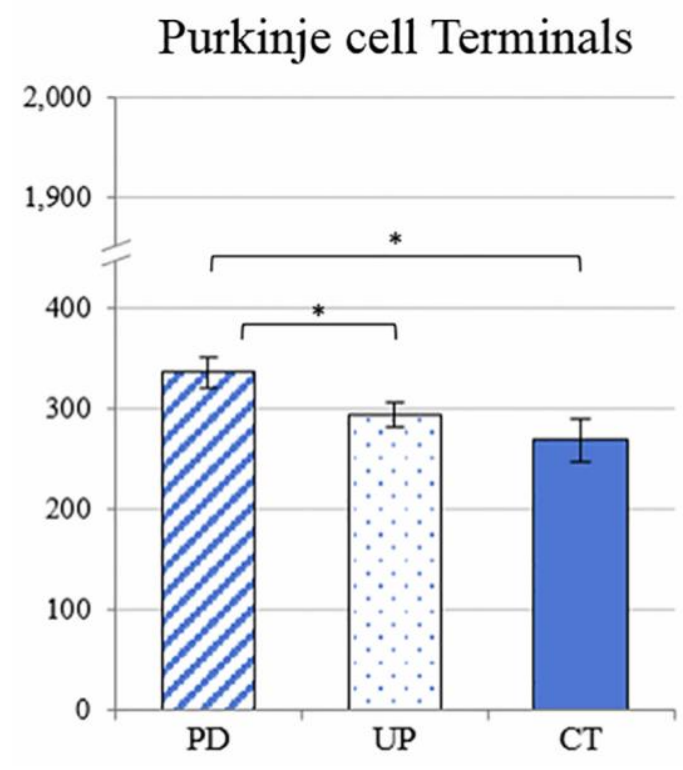

Figure 27. Number of Purkinje cell terminals apposing the soma of eyeblink projection neurons of the AIN.

The mean $( \pm \mathrm{SEM})$ number of Purkinje cell $(\mathrm{PC})$ terminals on the soma of eyeblink projection neurons of the AIN in the paired (PD), unpaired (UP), and control (CT) groups. The number of PC terminals in the PD group was significantly larger those in the UP and CT groups $(* \mathrm{P}<0.05)$. 


\section{Purkinje cell terminals make multiple synapses}

In order to establish the source of inhibitory synapses on eyeblink AIN neurons, the number of inhibitory PSDs that were apposed to PC terminals was measured as well as those that were not.

Inhibitory PSDs apposed to PC terminals were classified as PC synapses whereas those that were not were classified as interneuron synapses. We found that a single PC terminal apposed an average $3.21 \pm 0.13$ inhibitory synapses. There was a slightly larger number of synapses per PC terminal in the PD group compared to the other two groups but this difference was not significant. The average number of synapses per PC terminal was $3.27 \pm 0.11$ in the PD group, $3.15 \pm 0.10 \mu \mathrm{m}$ in the UP group, and $3.22 \pm 0.19 \mu \mathrm{m}$ in the CT group.

\section{Purkinje cell synapses}

Figure 28 shows the average number of PC synapses onto the somas of eyeblink AIN neurons in the PD, UP, and CT group respectively. The number of PC synapses was 1,102 \pm 33 in the PD group, $927 \pm 28$ in the UP group, and $867 \pm 40$ in the CT group. The number of PC synapses in the PD group was significantly larger than the UP $[F(1,133)=16.69, p<.0001]$ and CT $[F(1,74)$ $=13.05, p<.0001]$ group. This significant difference in the number of PC synapses found in the PD group represents a $20 \%$ increase compared to the UP group and a $27 \%$ increase compared to the CT group. The number of PC synapses in the UP group was not different from those found in the CT group. 


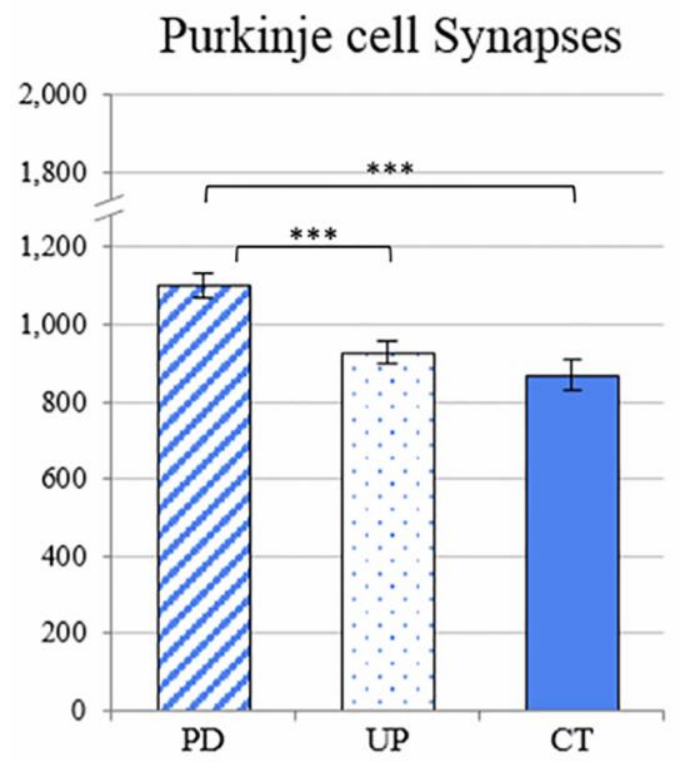

Figure 28. Number of Purkinje cell somatic synapses on eyeblink projection neurons of the AIN.

The mean $( \pm$ SEM) number of Purkinje cell $(\mathrm{PC})$ synapses on the soma of eyeblink projection neurons of the AIN in the paired (PD), unpaired (UP), and control (CT) groups. The number of PC somatic synapses in the PD group was significantly larger those in the UP and CT groups $(* * * \mathrm{P}<0.001)$.

\section{Interneuron synapses}

The average number of interneuron synapses onto the somas of eyeblink AIN neurons in the PD, UP, and CT group is shown in Figure 29. The number of interneuron synapses was $269 \pm 15$ in the PD group, $343 \pm 17$ in the UP group, and $256 \pm 22$ in the CT group. The number of interneuron synapses in the UP group was significantly larger than the $\operatorname{PD}[F(1,133)=10.76, p<$ $.001]$ and CT $[F(1,91)=5.76, p<.018]$ group. This significant difference in the number of interneuron synapses found in the UP group represents a $34 \%$ increase compared to the CT group and a $29 \%$ increase compared to the PD group. The number of interneuron synapses in the PD was not different from that found in the CT group. 


\section{Interneuron Synapses}

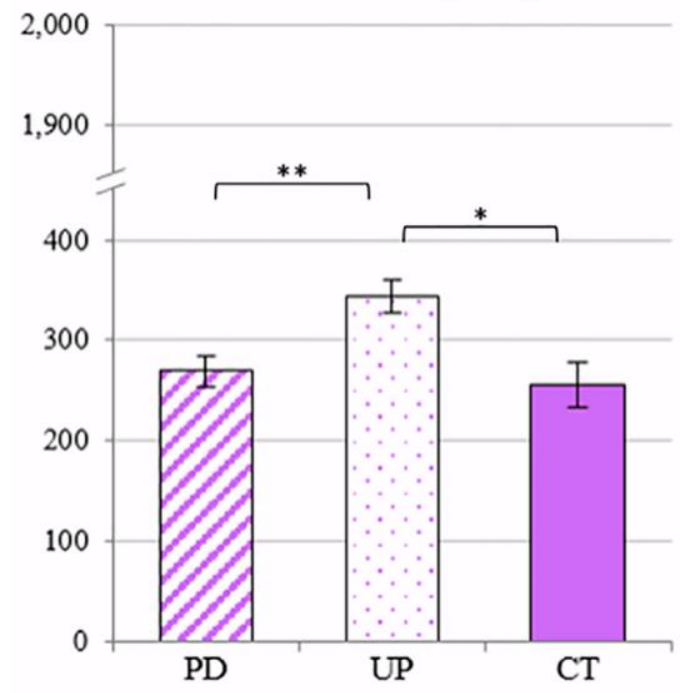

Figure 29. Number of interneuron somatic synapses on eyeblink projection neurons of the AIN.

The mean $( \pm$ SEM) number of interneuron synapses on the soma of eyeblink projection neurons of the AIN in the paired (PD), unpaired (UP), and control (CT) groups. The number of interneuron somatic synapses in the UP group was significantly larger than the PD and CT groups $(* \mathrm{P}<0.05, * * \mathrm{P}<0.01)$.

\section{Somatic area}

Figure 30 shows the average somatic area of eyeblink AIN neurons in the PD, UP and CT group. The average somatic area of eyeblink AIN neurons was $475 \pm 11 \mu \mathrm{m}^{2}$ in the PD group, $437 \pm 9 \mu \mathrm{m}^{2}$ in the UP group, and $395 \pm 11 \mu \mathrm{m}^{2}$ in the CT group. The somatic area in the PD group was significantly larger than the CT $[F(1,74)=12.43, p<.001]$ and UP $[F(1,133)=7.21$, $p<.008$ ] groups. The somatic area of neurons in the PD group was $20 \%$ larger than the CT group, and 9\% larger than the UP group. In addition, the somatic area of neurons in the UP group was $11 \%$ and significantly larger than the CT group $[F(1,91)=4.21, p<.043]$. 


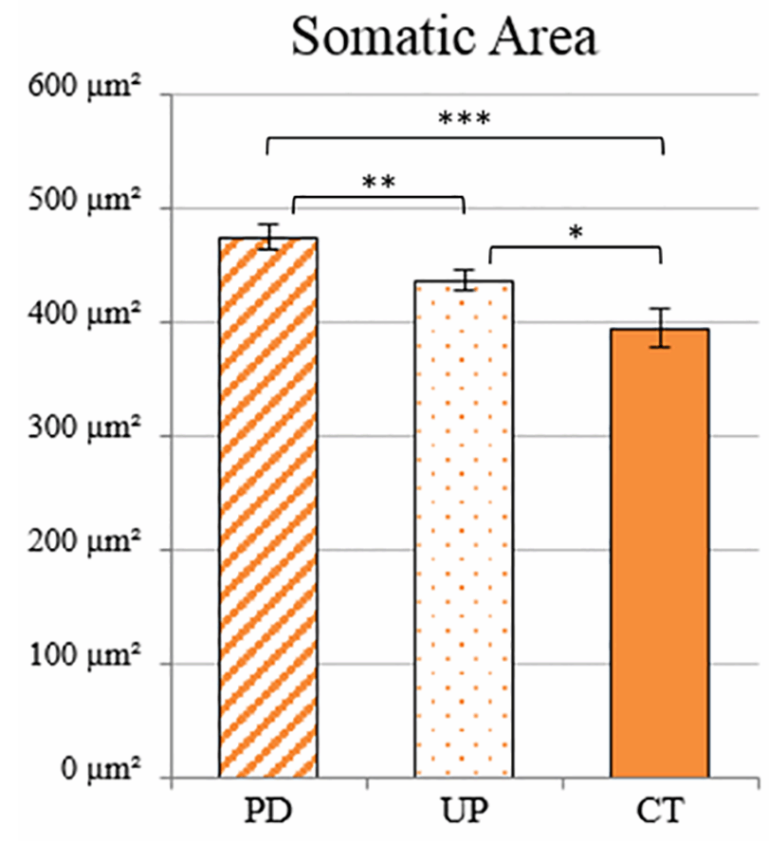

Figure 30. Somatic area $\left(\mu \mathrm{m}^{2}\right)$ of eyeblink projection neurons of the AIN. The mean $( \pm$ SEM) somatic area of neurons in the paired (PD), unpaired (UP), and control (CT) groups are shown. The somatic area in the PD group was significantly larger than the somatic area in the UP and CT groups $(* \mathrm{P}<0.05, * * \mathrm{P}<0.01, * * * \mathrm{P}<0.001)$.

\section{Size of presynaptic and postsynaptic densities}

The average diameter of excitatory and inhibitory PSDs and the average diameter of PC terminals in the PD, UP, and CT group was calculated. Initial analysis revealed that excitatory and inhibitory PSDs labeled with PSD95 and gephyrin respectively, were similar in size. The average diameter of excitatory PSDs across groups was $0.428 \pm 0.029 \mu \mathrm{m}$, and the average diameter of inhibitory PSDs across groups was $0.403 \pm 0.027 \mu \mathrm{m}$. The average diameter of excitatory PSDs was $0.413 \pm 0.019 \mu \mathrm{m}$ in the PD group, $0.441 \pm 0.017 \mu \mathrm{m}$ in the UP group, and $0.429 \pm 0.031 \mu \mathrm{m}$ in the CT group. The average diameter of inhibitory PSDs was $0.392 \pm 0.016$ $\mu \mathrm{m}$ in the PD group, $0.406 \pm 0.015 \mu \mathrm{m}$ in the UP group, and $0.411 \pm 0.026 \mu \mathrm{m}$ in the CT group. There were no significant differences in the diameter of PSDs across groups. 
The average diameter of PC terminals across groups was $1.153 \pm 0.152 \mu \mathrm{m}$. It is important to note here, that this value represents the diameter of the PC terminal that was apposing the somatic membrane and it is not reflective of the diameter of the PC bouton, which may be larger than the diameter apposing the soma. The average diameter of PC terminals was $1.202 \pm 0.046$ $\mu \mathrm{m}$ in the PD group, $1.153 \pm 0.037 \mu \mathrm{m}$ in the UP group, and $1.193 \pm 0.065 \mu \mathrm{m}$ in the CT group. There were not significant changes in diameter of PC terminals across groups.

\section{Discussion}

There were four major findings in this experiment. First, a significant increase in the number of somatic excitatory synapses was found on eyeblink AIN neurons of the PD group as a result of EBC. Second, a significant increase in the number of somatic inhibitory synapses originating from PCs was also found in the PD group. Third, a significant increase in the number of somatic inhibitory synapses originating from interneurons was found on eyeblink AIN neurons of the UP group after unpaired stimulus presentations. Fourth, the somatic surface area of the eyeblink AIN neurons was significantly correlated with the increase in the number of somatic synapses. Among these four major findings, the principal result was a coordinated increase in the number of somatic excitatory (presumably MFs) and inhibitory PC synapses on eyeblink premotor neurons of the AIN. To our knowledge, this is the first time that learning-specific synaptic changes have been observed in identified eyeblink premotor neurons of the AIN.

\section{Excitatory synapses}

We found an almost forty percent increase in the number of excitatory somatic synapses in eyeblink projection neurons in the PD group. This learning-specific increase in excitatory synapses probably originates from MF inputs that convey information about the tone-CS. 
However, there are three sources of excitatory inputs in the AIN: MFs, CFs, and recurrent collaterals. The source of excitatory inputs cannot be determined from our data and even though unlikely, we cannot rule out a possible contribution from CFs or recurrent collaterals to the increase in excitatory synapses found after EBC. This significant increase in excitatory synapses probably reflects structural changes taking place after prolonged tone-CS and airpuff-US paired training during the consolidation of CRs.

Evidence to support MF synaptogenesis after EBC comes from unit recordings, tract tracing, and neuroanatomical studies (McCormick and Thompson, 1984a; Berthier and Moore, 1990; Yang and Weisz, 1992; Kleim et al., 2002; Weeks et al., 2007; Boele et al., 2013). Single unit recordings from AIN neurons during (tone-CS and airpuff-US) paired training showed the response to the tone-CS gradually increased as training progressed. Eventually, the tone-CS evoked a large response in nuclear neurons that preceded the airpuff-US and that coincided with the execution of a CR (McCormick and Thompson, 1984a; Berthier and Moore, 1986). This evidence suggests that the strength of MF synapses that are activated by the tone-CS increase during EBC (Yang and Weisz, 1992).

Direct evidence of MF synaptogenesis during EBC comes from a recent study in adult mice (Boele et al., 2013). In this study Boele and colleagues injected an anterograde tract tracer, biotinylated dextran-amine, into the PN of three groups of mice that underwent different conditioning procedures. They found a significant increase in the number of MF terminals in the EBC group. This increase was the result of paired training because the group that received unpaired presentations of the same number of tone-CSs and airpuff-USs did not show an increase in number of MF varicosities. This study shows that MF axons can undergo substantial sprouting during EBC that results in a significant increase in the number of excitatory synapses in the AIN and other DCN (Boele et al., 2013).

Anatomical observations from EM studies suggest that excitatory synapses undergo a sequence of distinct structural changes during the early and late phases of EBC (Kleim et al., 2002; Weeks et al., 2007). The early phase refers to the period where the first CRs appear and the late phase refers to the period where CRs have been consolidated. An increase in the size of excitatory synapses in the rabbit AIN was seen during the early phase of EBC (Weeks et al., 2007). 
Whereas, a significant increase in the number of excitatory synapses was found in the rat AIN during the late phase of EBC (Kleim et al., 2002). The larger size of excitatory synapses observed during the early phase is probably caused by the potentiation of MF synapses and the insertion of additional AMPARs into the PSDwhereas the increase in number observed during the late phase probably reflects synaptogenesis of MF synapses caused by the division of larger synapses and axonal sprouting. These data support a learning process that may involve potentiation of excitatory synapses during the early phase that is followed by synaptogenesis during the late phase of EBC (Kleim et al., 2002; Weeks et al., 2007; Boele et al., 2013).

Support for the potentiation of MF synapses during the early phase of EBC comes from in vitro electrophysiology studies in the AIN. Unlike other CNS synapses, nuclear synapses do not potentiate by coincidence of presynaptic activation and postsynaptic depolarization. Instead, presynaptic activation must be followed by a period of postsynaptic hyperpolarization and a subsequent rebound burst of action potentials. In fact, potentiation of MF synapses is achieved using an induction protocol that resembles the sequence of tone-activated MFs and airpuffactivated PCs that occur during EBC (Pugh and Raman, 2006, 2008).

Induction of LTP at excitatory MF synapses requires a precisely timed sequence of MF and PC activation. Optimal LTP induction is achieved when MF stimulation precedes PC stimulation by 250-400 ms (Pugh and Raman, 2008). As MF stimulation moves away from this optimal 250400 ms window, LTP becomes weaker and eventually reverses to LTD (Pugh and Raman, 2008; Zheng and Raman, 2010). This optimal window becomes particularly relevant in EBC because importantly, 250-400 ms is the optimal interval between the tone-CS and airpuff-US for CR acquisition (Smith et al., 1969). This evidence suggests that nuclear neurons are particularly tuned to sort afferent inputs and selectively enhance those that fall in this precise time window.

Selective potentiation of MF inputs is achieved by the activation of NMDARs and Ca2+ influx that act as a priming mechanism to identify recently activated synapses (Person and Raman, 2010). Potentiation of primed synapses is accomplished by the action of the airpuff-activated PCs that produce an hyperpolarization and rebound bursts of action potentials on the postsynaptic neuron. In particular, the drop and rise in intracellular $\mathrm{Ca} 2+$ triggers a signal cascade responsible for the potentiation of recently activated synapses (Pugh and Raman, 2008). 
In this manner, airpuff-activated PCs selectively regulate plasticity of MF afferent inputs on eyeblink projection neurons.

Support for NMDAR-dependent potentiation of MF synapses during the early phase of EBC comes from an in vivo study in rabbits. Infusion of an NMDAR antagonist in the AIN impaired the formation of CRs, but had no effect when the antagonist was infused after the CRs were acquired (Chen and Steinmetz, 2000). In addition, support for synaptogenesis during the late phase of EBC comes from another in vivo study in rabbits. Infusion of anisomycin, a protein synthesis inhibitor required for synaptogenesis, blocked the formation of CRs but had no effect when the inhibitor was infused after the CRs were acquired (Bracha et al., 1998).

Our findings provide evidence of an increase in the number of excitatory somatic synapses as a result of learning that support synaptogenesis (presumably from MF) during the late phase of EBC. Most importantly, synaptic plasticity was observed on eyeblink premotor neurons of the AIN that constitute a functional distinct group of neurons. These eyeblink projection neurons can regulate eyeblink responses by direct activation of the $\mathrm{RN}$ which in turn controls motoneurons innervating the OOM. To our knowledge, this is the first time that learningspecific changes can be linked to AIN neurons controlling the execution of the CR. Our data along with the evidence presented above support a learning process in the AIN that involves potentiation of MF synapses during the early phase that is followed by synaptogenesis during the late phase of EBC. This process appears to be regulated by the action of PCs that are activated by the airpuff-US that selectively potentiates preceding MF signals that can be used as predictors. 


\section{Purkinje cell synapses}

In addition to an increase in the number of excitatory synapses, we found a $\sim 25 \%$ increase in the number of somatic PC synapses on eyeblink AIN neurons in the PD group. This learningspecific increase in the number of PC synapses was unexpected and to our knowledge, unprecedented. This finding was unexpected because the prevailing cerebellar models of motor learning predict a decrease rather than an increase in PC inputs (Albus, 1971; Eccles, 1973; Ito, 2006). It is intriguing that others studies of the AIN found changes in excitatory but not inhibitory synapses after EBC (Kleim et al., 2002; Weeks et al., 2007). A possible reason for this lack of evidence is that unlike MF synapses, PC synapses are located preferentially on the soma and proximal dendrites of nuclear neurons and detection of these changes may be sensitive the experimental design. Another reason is that MF sprouting may be extensive and distributed (Boele et al., 2013), whereas PC synaptogenesis may be restricted and confined to specific neurons. Perhaps more probable is the fact that synaptic plasticity during EBC takes place in a discrete number of neurons and can be difficult to detect when examining a large region such as the AIN comprised of multiple types of neurons performing different functions.

Purkinje cell synapses are highly plastic and can undergo different types of activity-dependent changes such as LTP and LTD (Sastry et al., 1997; Aizenman et al., 1998; Aizenman et al., 2000; Telgkamp and Raman, 2002). Purkinje cell LTP can be induced by high frequency stimulation that successfully hyperpolarizes and, once it is relieved, triggers a rebound burst of action potentials on its target nuclear cell. This type of LTP is also Ca2+dependent and the number of action potentials elicited after the rebound greatly influences the magnitude of the potentiation (Sastry et al., 1997; Aizenman et al., 2000). On the other hand, low frequency stimulation that is insufficient to produce rebound bursts of action potentials and their associated increase in $\mathrm{Ca} 2+$ induces LTD. In short, PC synapses that are active before the period that successfully triggers a rebound burst of action potentials on the postsynaptic nuclear neuron will undergo LTP and those that are not will undergo LTD (Morishita and Sastry, 1993; Sastry et al., 1997; Aizenman et al., 2000; Ouardouz and Sastry, 2000). In this manner, a rebound burst generated by the airpuff-activated PCs may be capable of triggering LTP at the weaker toneactivated PC synapses. 
Nuclear cells receive the majority of their synaptic inputs from dozens of inhibitory PCs. However, most of these inputs originate from only a few PCs. In fact, many investigators have described that a single PC axon can innervate a large number of neurons but strategically surround the soma of specific nuclear cells (Chan-Palay, 1973c, b; Palkovits et al., 1977; Sugihara et al., 2009). During EBC, the tone-CS and airpuff-US activate specific PCs that produce an inhibitory response on their target nuclear neurons that is proportional to their synaptic weight (Bengtsson et al., 2011). For this reason, the strength of the hyperpolarization and subsequent rebound burst depends on which PCs are activated. Let's think for a minute about the functional relevance of this disparity in PC innervation and its possible role in motor control. A PC receives inputs from only one CF, however, a single CF innervates $\sim 7$ PCs (in the rat) (Schild, 1970; Delhaye-Bouchaud et al., 1985; Sugihara et al., 2001). Consequently, stimulation of a single CF can potentially activate 7 PCs in different eyeblink microzones that converge on the same nuclear neuron (Blenkinsop and Lang, 2011). In addition to CFs, MF signals are conveyed to each of these PCs by thousands of PF synapses from granule cells. Therefore, information about the tone-CS is conveyed by PF synapses that probably contact only a subset of PCs located in different eyeblink microzones. Consequently, PCs that share the same postsynaptic nuclear neuron may receive inputs from the tone-CS or the airpuff-US exclusively, or from both. The particular synaptic weight of each of these PCs on the postsynaptic neuron may be dependent on their past synaptic history. During paired delay EBC, the tone-CS (by way of PFs) activates PCs that probably produce a weak hyperpolarization on the postsynaptic neuron that fails to trigger a rebound burst. Whereas the airpuff-US (by way of CFs) probably activates dozens of PCs in multiple eyeblink microzones that together produce a strong hyperpolarization and rebound burst of action potentials on the postsynaptic neuron. Therefore, the hyperpolarization and rebound burst elicited by the airpuff-US acts as an instructor signal on the postsynaptic neuron that triggers the potentiation of those preceding PC synapses that were activated by the tone-CS. In turn, the synaptic weight of individual PCs can be modulated to enhance synapses from those PCs that are activated by the tone-CS that can be used as predictors of the airpuff-US.

This appears to be the case during EBC, where PCs that are activated by the tone-CS evoke weak responses on their target nuclear neurons initially but become stronger as paired training 
progresses and CRs are acquired (Rasmussen et al., 2008). These results provide evidence of a gradual increase in PC synaptic input during EBC that supports the significant increase in the number of PC synapses observed in our study. In addition, PC responses to the tone-CS gradually shifts during EBC. Initially, PF activated by the tone-CS produce small and irregular simple spike responses on their postsynaptic PCs. As training progresses, simple spike responses gradually shift to occur at the time of the tone onset (Jirenhed et al., 2007; Rasmussen et al., 2008; Svensson et al., 2010; Hesslow et al., 2013). Eventually, PF can elicit a synchronized burst of simple spikes that is followed by a pause in PC firing, probably mediated by inhibitory interneurons of the cerebellar cortex (stellate and basket cells). Our data, along with this evidence from the literature support a learning mechanism that involves PC synaptic plasticity on nuclear neurons and also on the cerebellar cortex.

\section{Interneuron synapses}

We found a $\sim 30 \%$ increase in the number of interneuron somatic synapses on eyeblink AIN neurons in the UP group compared to the CT and PD groups. This finding was unexpected and provides evidence of synaptic plasticity after UP stimulus presentations mediated by local inhibitory interneurons. The behavioral effects of UP training has long been recognized (Baker and Mackintosh, 1977; Allen et al., 2002; Linden et al., 1997; Baker et al., 2003; Bonardi and Ong, 2003). Subjects preexposed to UP presentations show a significant delay or retardation in the rate of subsequent CR acquisition also known as "learned irrelevance" (Bennett et al., 1995; Rush et al., 2001; Allen et al., 2002). The retardation effect on CR acquisition suggests that UP presentations produce activity-dependent changes that interfere with subsequent learning.

The role and function of AIN interneurons during EBC is largely unknown. In Chapter 3, we described 3 types of local interneurons that make direct connections with excitatory eyeblink projection neurons. In turn, these interneurons receive recurrent collaterals from projection neurons forming an inhibitory feedback loop (Matsushita and Iwahori, 1971b). Feedback inhibition in the AIN has not been studied, however, evidence from early in vivo single-unit 
recordings suggests that it may attenuate or even shunt ongoing activation on the postsynaptic neuron (Tarnecki and Zurawska, 1989).

Support for an increase in feedback inhibition during UP training comes from in vivo unit recordings in rabbits and rats (Berthier and Moore, 1990; Campolattaro et al., 2011). Recordings from AIN neurons that are activated by the US and can elicit an eyeblink when stimulated showed a significant increase in excitability during UP training. Particularly, the activity rate and sensitivity to the CS increased when the US was introduced. However, over time and after numerous unpaired presentations, the firing rate of these neurons returned to basal levels. These findings suggest that an inhibitory feedback mechanism is probably in place to counteract the increased excitability produced by the US. In this manner, activation of eyeblink projection neurons can activate local interneurons which by inhibitory feedback can decrease excitation.

Indeed, an increase in interneurons synapses can explain the normalization in the firing rate of nuclear neurons and also the retardation effect on subsequent learning. Increased feedback inhibition would interrupt rebound bursts elicited by the airpuff-US and consequently attenuate $\mathrm{Ca} 2+$ fluctuations. In fact, fluctuations in $\mathrm{Ca} 2+$ trigger signal cascades that mediate neuronal excitability and also synaptic plasticity. An increase in feedback inhibition will interfere with subsequent learning by attenuating or shortening the rebound burst and its associated $\mathrm{Ca} 2+$ fluctuations necessary for the induction of MFs and PCs LTP. Consequently, an increased inhibitory feedback inputs may delay the formation of CRs during EBC.

Increased excitability in response to the US can be highly adaptive and probably mediates synaptic plasticity of MF and PC synapses during paired training in EBC. In fact, a learningspecific increase in neuronal excitability has been reported in numerous behavioral models (Schreurs et al., 1991; Schreurs et al., 1997; Schreurs et al., 1998; Wang and Schreurs, 2006; Belmeguenai et al., 2010; Mozzachiodi and Byrne, 2010; Wang and Schreurs, 2010). In particular, increased excitability of nuclear neurons has been reported after different stimulation protocols (Aizenman and Linden, 2000; Zhang et al., 2004; Molineux et al., 2008; Tadayonnejad et al., 2010). Evidence suggests that increased excitability is permissive and probably necessary for synaptic plasticity. In addition, neuronal excitability can be global or confined to a specific region of the postsynaptic membrane to act selectively on local inputs. However, this increase in 
excitability could become maladaptive if sustained for too long. Feedback inhibition probably regulates the period for which a nuclear neuron is allow to sustain increased excitability. By limiting the duration, feedback inhibition confines activity-dependent plasticity to a specific critical period before the neuron returns to its homeostatic state.

\section{Somatic surface area}

The fourth major finding in this study was an increase in somatic surface area of eyeblink AIN neurons that was significantly correlated with the number of somatic synapses. This finding suggests that synaptogenesis can also induce proportional changes in the surface area of the postsynaptic neuron. Another possibility is that the increase in somatic surface area was caused by the transneuronal viral tracer, PRV. However, this is unlikely because careful analysis of the electrophysiological and synaptic properties of neurons that were infected with PRV for a longer period than the neurons studied here showed that they were healthy and undistinguishable from uninfected control neurons (Smith et al., 2000; McCarthy et al., 2009). In addition, if viral infection was responsible for the increase in somatic surface area, it would have affected all neurons. However, the somatic surface area had a significant effect of group, suggesting that this change was associated with the experimental condition rather than the viral tracer or any other variable.

Even though surprising, activity-dependent changes in the somatic area of neurons is not uncommon and it has been reported in a number of different systems. Experimental procedures such as treadmill running, stress, lactation, captivity or even aging can produce a significant increase or decrease in the somatic surface area of motor, hypothalamic, hippocampal, and cerebral neurons (Salm et al., 1988; Modney and Hatton, 1989; Murphy et al., 1990; Miyata et al., 1994; El Majdoubi et al., 1997; Nakano et al., 1997; Soghomonian et al., 2010; Ilha et al., 2011; Stigger et al., 2011; Freas et al., 2013). The change in the somatic area of these neurons was accompanied by a corresponding change in the number of synapses. In addition, these changes in somatic area and number of synapses were the opposite when another experimental condition was used. 
This evidence together with our results suggests that structural changes associated with synapse remodeling are not unilateral. To the contrary, the postsynaptic neuron is the target of thousands of inputs that are plastic, and as such it is required to undergo comparable structural alterations to accommodate them. In this manner, the surface area of the postsynaptic neuron is continuously adjusting to its synaptic inputs. Inevitably these changes would be more profound in neurons that are involved in learning.

\section{Purkinje cell terminals}

Another important finding was that a single PC terminal made on average 3.21 synapses. Purkinje cell terminals making multiple synapses have been previously reported in various species (Chan-Palay, 1971, 1973c; Telgkamp et al., 2004; Hoebeek et al., 2010). However, it was not clear to what extend and how common this feature was.

An EM study analyzing the ultrastructure of 10 PC boutons in young mice reported $\sim 9$ synapses per terminal (Telgkamp et al., 2004). This value probably does not reflect the population average because of the small number of boutons examined, however, it suggests that multiple synapses are a common feature of PC terminals. In addition, it may provide some evidence of developmental changes in the ultrastructure of PC terminals leading to refinement and a decrease in the number of synapses per bouton.

Additional support for PC terminals making multiple synapses comes from a study using a combination of EM and light microscopy in the cat (Palkovits et al., 1977). Based on EM observations, a single PC was estimated to make $\sim 474$ synapses. However, observations of Golgi stained PCs under the light microscope revealed that a single PC axon gave rise to $~ 100$ 200 swellings. Based on this findings, a single PC bouton was estimated to make on average $\sim 2.7$ synapses. These results are strikingly similar to the $\sim 3.21$ PC synapses per bouton found in our study. Additional support comes from a study in rats that also reconstructed the entire trajectory of individual PC axons and found that a single PC gave rise to $~ 120$ swellings, consistent with those found in the cat (Sugihara et al., 2009). Taken together this evidence 
suggests that multiple synapses are a common feature of PC boutons and conserved across species (Palkovits et al., 1977; Telgkamp et al., 2004; Pugh and Raman, 2005; Hoebeek et al., 2008).

\section{Limitations}

\section{Viability of PRV infected AIN neurons}

An important point to consider is how PRV infection could affect eyeblink AIN neurons. The viral properties that make PRV a valuable tool for transsynaptic tracing also can lead to apoptosis and neuronal death. However, a neuron appears to be healthy and asymptomatic for a window of at least 16 hours after PRV infection (McCarthy et al., 2009). In addition, the viability and synaptic integrity of PRV-labeled neurons was shown in an electrophysiology study (Smith et al., 2000). Whole-cell patch-clamp recordings 5 days (120 hours) after PRV injections showed that the resting membrane potential, the input resistance, and the spontaneous firing frequency of PRV-labeled neurons were undistinguishable from uninfected control neurons. In addition, the properties of evoked inhibitory and excitatory postsynaptic currents revealed that the synaptic inputs in PRV-labeled neurons remained intact (Smith et al., 2000). This evidence suggests that even though PRV infection would eventually cause neuronal deterioration and death, at the time studied here ( $4 \frac{1}{2}$ days), the synaptic and electrophysiological properties of eyeblink AIN neurons would presumably be comparable to the properties of uninfected AIN neurons. 


\section{Number of subjects}

It is important to note that one subject in the CT group was excluded from the synaptic data analysis because it showed early symptoms of infection and it was sacrificed 4 days after PRV injection (Table 3-1). Even though PRV infection was pronounced in the brain stem of this subject, there were not PRV-labeled neurons in the AIN. Synaptic data for the CT group derives from only one subject, and this should be taken into consideration when interpreting these data. This could present a problem if there were differences across subjects that are independent of the behavioral conditions. However, statistical analysis suggests that this is not the case. Statistical analysis revealed that there was no random effect introduced by the subject, suggesting that any differences can be attributed to the experimental conditions. More importantly, analysis of the differences found here as a function of the number of subjects in the PD and UP groups, rather than as a function of the number of neurons, still showed them to be statistically significant.

\section{Coronal sections and directionality bias}

A possible source of bias in this study was the use of coronal sections to estimate the number of somatic synapses. Stereology provides meaningful quantitative descriptions of the geometry of 3D structures from measurements that are made on 2D images (Mayhew, 1979; West, 1999). Using stereology techniques it is possible to reconstruct the size and distribution of synapses and to relate their size and number to an appropriate reference, such as a surface area. Biological structures with no preferred orientation in 3D space are isotropic, whereas structures that have a preferred orientation are anisotropic (Russ and Dehoff, 2000; West, 2012). Measures of length and surface of anisotropic structures are sensitive to the orientation plane, whereas counts or numerical measures are not. The use of sections with multiple orientation planes is desired for unbiased estimates of structures that have a preferred orientation. In this study, estimates of the size of PSDs and the surface area of eyeblink AIN could have been sensitive to the orientation plane if these structures are anisotropic. For this reason, a possible source of bias was introduced by the use of coronal sections. However, the source of bias would be systematic and affect all neurons and in all groups equally. 
It is important to note here that the main goal of this study was to make sensitive comparisons across groups examining the effect of EBC on somatic synapses of eyeblink AIN neurons. In order to determine the effect of the experimental variable, all other parameters were kept identical. Using multiple orientation planes would have introduced additional variables that could have confound the results. Consequently, the use of a single orientation plane allowed for sensitive comparisons across subjects and groups but could have introduced a systematic bias in synaptic estimates that may deviate from the population average. 


\section{Chapter 5. General Discussion}

\section{Main findings}

The main goal of this dissertation was to identify eyeblink projection neurons of the AIN and to examine learning-specific changes in excitatory and inhibitory somatic synapses after EBC. In order to identify the specific subset of AIN neurons involved in EBC, a retrograde transneuronal viral tracer, PRV was injected into the $O O M$ of the rabbit to reveal hierarchical chains of synaptically connected neurons involved in the eyeblink response.

The first experiment was designed to provide a complete delineation of the rabbit eyeblink premotor pathway and to characterize the multiple neuronal types in the AIN that are involved in EBC and their possible interactions (Chapter 3). Relevant structures involved in the generation or modulation of the rabbit eyeblink response were identified and possible sites of plasticity were revealed. Eyeblink premotor neurons of the AIN were further characterized based on their neurotransmitter immunoreactivity and showed that glutamatergic eyeblink projection neurons are modulated by three different types of inhibitory interneurons that form a functional eyeblink microcomplex.

In Chapter 4 the effect of EBC in the number of excitatory and inhibitory somatic synapses on eyeblink projection neurons of the AIN was examined. A coordinated increase in the number of excitatory and inhibitory PC somatic synapses was observed in subjects that acquired eyeblink conditioned responses. These results support a parallel and correlated mechanism of cerebellar learning mediated by excitatory (presumably from MFs) and inhibitory PCs. In contrast, control subjects that received unpaired stimulus presentations showed an increase in the number of inhibitory somatic synapses from local interneurons, suggesting a possible role of feedback inhibition that may also explain the retardation effect characteristic of unpaired stimulus preexposure. Another important finding was that the somatic surface area of eyeblink projection 
neurons was highly correlated with the number of somatic synapses; suggesting that synaptic remodeling is a bilateral process that entails proportional structural alterations on the postsynaptic neuron. To our knowledge, this is the first time that the identity and function of specific nuclear neurons and their associated synaptic changes during learning can be linked to a behavior.

\section{Eyeblink premotor neurons of the anterior interpositus nucleus}

The role of eyeblink premotor neurons of the AIN is to execute adaptive eyeblinks in response to somatosensory and nociceptor afferent inputs. Results from Experiment 1 and $\mathbf{2}$ (presented in Chapter 3 and 4, respectively) revealed $\sim 20-30$ eyeblink projection neurons in the AIN, immunoreactive for glutamate that project to the RN. This number was estimated by the maximum number of third order eyeblink AIN neurons found at $4 \frac{1}{2} 2$ days that were immunoreactive for glutamate. Our data shows that these $\sim 20-30$ eyeblink projection neurons are directly modulated by 3 types of local interneurons (GABAergic, glycinergic, and mixed GABA/glycinergic). Evidence from other studies suggests that mixed GABA/glycinergic interneurons can adjust the neurotransmitter ratio in their terminals and consequently modify the response kinetics on the postsynaptic neuron (Crook et al., 2006; Seal and Edwards, 2006; Dresbach et al., 2008). It is not clear if the interneurons identified in our study correspond to 3 functional distinct types that perform different tasks or if they represent the spectrum of a single class. However, interneurons appear to play an important role in the modulation of the output from the AIN. In this regard, eyeblink projection neurons execute precisely timed eyeblink CRs by integrating excitatory input from MF and $\mathrm{CF}$, and inhibitory inputs from PCs and local interneurons.

The number of somatic synapses found in our study was 1,460 in the CT group (Experiment 2) which is in good agreement with the $\sim 1,800$ somatic synapses estimated on large AIN neurons of the cat (Palkovits et al., 1977). About $\sim 22 \%$ of somatic synapses were excitatory and $\sim 78 \%$ were inhibitory corresponding to $\sim 60 \%$ PC somatic synapses and $\sim 18 \%$ interneuron somatic synapses. 
These results are consistent with the percentage of PC and interneuron synapses found in other studies (Wassef et al., 1986; Chen and Hillman, 1993; De Zeeuw and Berrebi, 1995; Baurle and Grusser-Cornehls, 1997; Sultan et al., 2002). However, even though in our study excitatory synapses were a small percentage of somatic synapses, they were clearly a substantial source of somatic inputs and more abundant than previously reported (Chan-Palay, 1973c; De Zeeuw and Berrebi, 1995).

Most of our knowledge about the synaptic inputs on nuclear neurons derives from EM studies that classified excitatory and inhibitory synapses based on the morphological features of presynaptic vesicles (Uchizono, 1965) and on the aggregation of electro-dense material on the postsynaptic membrane, PSD (Gray, 1959, 1969). However, a large proportion of synapses cannot be classified with certainty based on these parameters. In addition, it has been reported that these morphological features do not always align with synaptic function such as the neurotransmitter content of presynaptic terminals and the type of receptors expressed on the PSD (Clements et al., 1990; van den Pol, 1991; Klemann and Roubos, 2011). Evidence from Golgi stain and tracing studies indicate that MFs and CFs do indeed contact the soma of nuclear neurons (Matsushita and Iwahori, 1971a; Ikeda and Matsushita, 1973; Matsushita and Ikeda, 1976; Shinoda et al., 2000). The fact that only sparse excitatory synapses were observed in some EM studies suggests that somatic excitatory synapses may exhibit the morphological features associated with inhibitory synapses.

Indeed, silent synapses can show the same morphological features as inhibitory synapses. Because silent synapses are dormant and express only NMDARs, the aggregation of electrodense material in the PSD is light and appears symmetric under the EM. Even though they are dormant, silent synapses can undergo activity-dependent potentiation by insertion AMPARs into the PSD. In addition, this process is dynamic and mature synapses can also become silent. Silent synapses are not uncommon; they are most frequent during development but found regularly in the adult brain. Indeed, silent synapses constitute over $60 \%$ of the synapses in the cerebellar cortex (PF-PC synapses) (Wang et al., 2000; Isope and Barbour, 2002). Our results suggest that somatic excitatory synapses are not as sparse as previously thought (Chan-Palay, 1973c; De Zeeuw and Berrebi, 1995). In addition, these results suggest that excitatory inputs on 
AIN projection neurons may be particularly malleable. Excitatory inputs appear to be subject to profound and bidirectional changes. Importantly, our data suggests that plasticity of excitatory synapses is one of the neuronal mechanisms underlying learning in the AIN.

\section{Significance}

Eyeblink projection neurons of the AIN are glutamate-ir (Chapter 3) and have been shown to activate contralateral $\mathrm{RN}$ neurons that control motoneurons in the $7 \mathrm{~N}$ and other brainstem nuclei to elicit an eyeblink response. The experimental design used in this Dissertation allowed for direct identification of AIN eyeblink projection neurons. These PRV-labeled neurons are synaptically connected to the pathway involved in the eyeblink response. In addition, the timedependent properties of PRV spread in combination with neurotransmitter immunolabeling allowed us to identify glutamatergic eyeblink projection neurons of the AIN and to study learning-specific changes on those neurons. The fact that only eyeblink projection neurons were analyzed in this study provides a level of significance that is unprecedented. Unlike other studies that measured the effect of EBC in the number or size of excitatory and inhibitory synapses in the AIN, here the effect of EBC was studied on eyeblink projection neurons exclusively. The identity of the postsynaptic AIN neurons is an important factor in assessing learning-specific changes as a function of EBC for two main reasons. First, only a small fraction of AIN neurons are involved in EBC. The AIN is a large nuclei containing over 5,000 neurons involved in the control and modulation of over 300 skeletal muscles. For this reason, most of the synaptic measurements would originate from AIN neurons that are not involved in the eyeblink response and consequently, the results derived from these measurements would be in its majority, unrelated to learning. Second, because there are 3 main types of nuclear neurons, the identity of AIN neurons is also important. It is possible that glutamatergic projection neurons, small GABAergic neurons that project to the IO, and local interneuron may all be involved in EBC. However, because they perform different functions, synaptic changes on different AIN neurons are probably type-specific. The power of the results presented in this Dissertation derives from the fact that the specific AIN neurons involved in the eyeblink premotor pathway were directly 
identified. Most importantly, because eyeblink premotor neurons were identified, we were able to determine the effect of $\mathrm{EBC}$ in the number of somatic synapses.

\section{The role of the anterior interpositus in eyeblink conditioning}

In the early 1980s a series of ground breaking studies identified the ipsilateral cerebellum as the engram of learning in EBC (Lincoln et al., 1982; McCormick et al., 1982b; Lavond et al., 1984; McCormick and Thompson, 1984a). However, there is an ongoing controversy about where in the cerebellum learning takes place, the AIN or the cerebellar cortex. The DCN and in the case of EBC, the $\mathrm{AIN}$ is the last processing station; it integrates extracerebellar and intracerebellar signals to execute the only and final cerebellar output. In this regard, large glutamatergic projection neurons constitute the last processing station of the AIN; they integrate excitatory and inhibitory inputs to produce the ultimate cerebellar output. Even more, cerebellar commands are carried out by glutamatergic projection neurons and cerebellar-dependent eyeblink responses and eyeblink CRs are executed by glutamatergic eyeblink projection neurons of the AIN.

In Experiment 1, glutamatergic eyeblink projection neurons of the AIN were identified using a retrograde transsynaptic tracer in (Gonzalez-Joekes and Schreurs, 2012). In Experiment 2, the effect of EBC on the number of excitatory and inhibitory somatic synapses was studied on identified AIN neurons which are responsible for the execution of cerebellar eyeblink responses and eyeblink CRs. The results in Experiment 2 revealed several significant findings and some were unexpected. Most importantly, some of our findings were unprecedented and provide new insight on the possible mechanisms of learning.

A summery of the major findings in Experiment 2 is illustrated in Figure 31. The neuronal pathways involved in EBC and the effect of paired and unpaired training on the number of 
somatic synapses on eyeblink projection neurons of the AIN in the CT, PD, and UP groups is shown in Figure 31 A, B, and $\mathbf{C}$ respectively.

The afferent pathway that conveys information about the tone-CS to the cerebellum is depicted in blue and the afferent pathway that conveys information about the airpuff-US is depicted in red. Information about the tone-CS and airpuff-US is integrated on eyeblink projection neurons (shown in green) that form the final output from the cerebellum. Eyeblink projection neurons of the AIN integrate extracerebellar and intracerebellar signal to generate precisely timed commands. The basal state of afferent inputs in the AIN is represented by the CT group in Figure 31 A. Subjects in the CT group were exposed to the same routine and handling as the PD and UP group, however the CT group did not receive any tone-CS or airpuff-US.

The major finding in this study was a coordinated increase in the number of excitatory (presumably MFs) and inhibitory PC somatic synapses in the PD group as a result of EBC.

Figure 31 B illustrates the synaptic changes taking place on eyeblink projection neurons (green) as a result of paired delay training in the PD group. An increase in the number of excitatory synapses was not surprising and evidence suggests that this learning mechanism is mediated by synaptogenesis of MFs that convey information about the tone-CS (Kleim et al., 2002; Boele et al., 2013). On the other hand, an increase in the number of PC somatic synapses was novel and unexpected. The role of PCs in EBC and the possible synaptic mechanisms underlying learning are discussed in the next section.

Another important finding in this study was an increase in the number of interneuron somatic synapses as a result of unpaired training. Figure $31 \mathrm{C}$ illustrates the synaptic changes taking place on eyeblink projection neurons (green) as a result of unpaired training in the UP group. An increase in the number of interneuron synapses was surprising. However, the detrimental effect of unpaired exposure on subsequent learning is well-known. Our findings provide evidence of long-lasting synaptic changes in the AIN as a result of unpaired training. In addition, an increase in the number of interneuron synapses may also explain the neuronal mechanism underlying a common phenomenon known as "learned irrelevance" (see the discussion section in Chapter 4 for more details). 

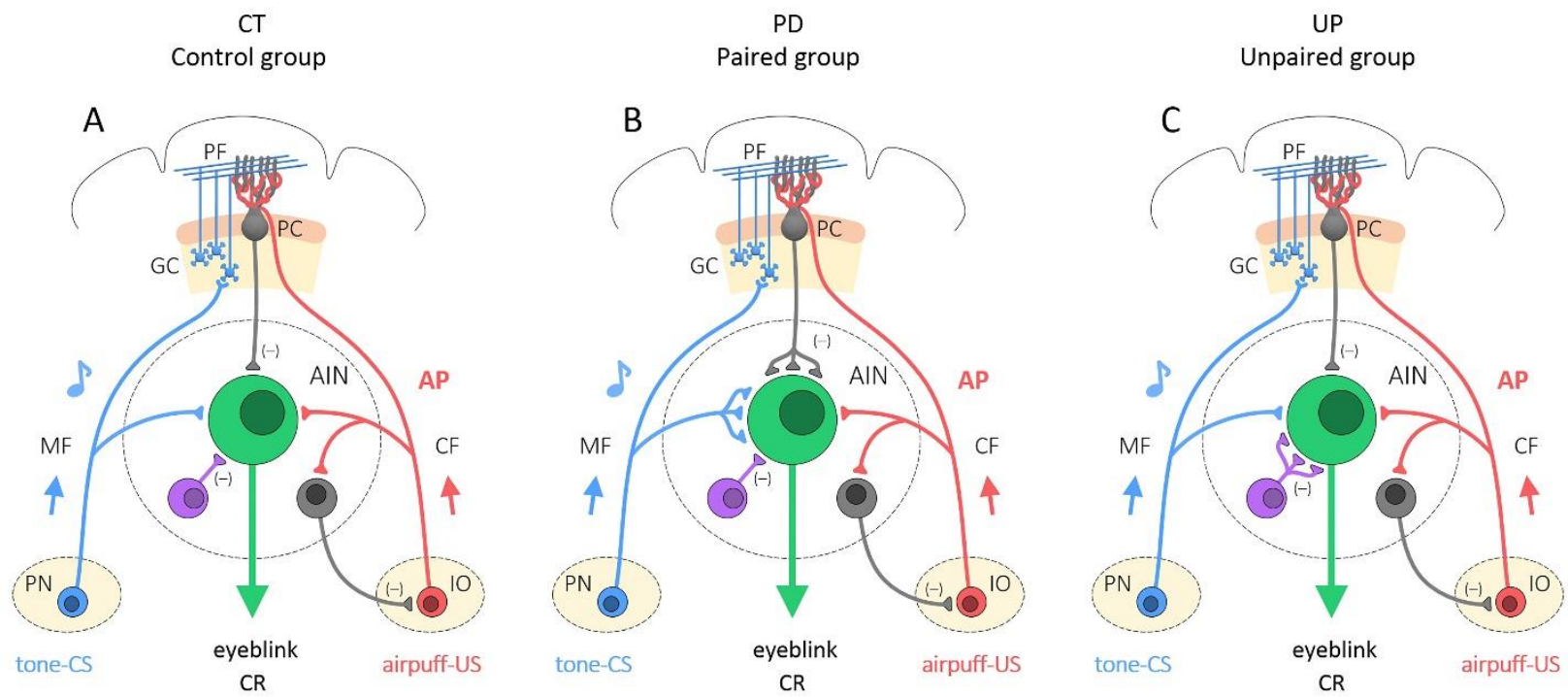

Figure 31. Synaptic plasticity on anterior interpositus eyeblink projection neurons.

The effect of paired and unpaired training on the number of somatic synapses in: A, control (CT); $\mathbf{B}$, paired (PD); and $\mathbf{C}$, unpaired (UP) groups respectively.

\section{Learning Mechanisms in the Cerebellum}

Data presented in this Dissertation provide direct evidence of synaptic plasticity on eyeblink projection neurons of the AIN as a result of EBC and UP training. In addition, these results provide valuable information about the neuronal mechanisms underlying learning.

Based on the results presented here and the current state of knowledge about cerebellar learning a possible model of cerebellar learning was formulated. Figure 32 shows a possible mechanism of learning in the cerebellum. Figure 32 shows a circuit diagram of the essential cerebellar pathways involved in EBC and the possible synaptic changes underlying learning. Figure $32 \mathrm{~A}$ shows a circuit diagram at the beginning of paired training during EBC and before an association between the tone-CS and the airpuff-US is formed. Figure 32 B shows the early phase of EBC where the first CRs appear. 
The formation of the first CRs is probably mediated by LTP of MF synapses that convey information about the tone-CS. In addition, in our model of cerebellar learning, PC synapses that convey information about the tone-CS are also potentiated, shown in Figure 32 B. Figure 32 C shows the late phase of EBC where CRs are consolidated by prolonged paired training. The consolidation of CRs is mediated by synapse formation of previously potentiated synapses. Previously potentiated excitatory MF synapses divide and form new synapses. In the same manner, previously potentiated PC synapses undergoe synaptogenesis (Figure 32 C).

The first step of learning takes place at the beginning of paired delay training before an association between the stimuli is formed (Figure 32 A). The first step of learning is probably accomplished by an increase in membrane excitability of eyeblink AIN neurons. This increase in membrane excitability is elicited by the action of PCs that are activated by the airpuff-US. In this manner, activation of CFs by the airpuff-US produces a strong and synchronized activation of many PCs distributed across the cerebellar cortex in multiple eyeblink microzones. These airpuff-activated PCs converge (roughly) on the same postsynaptic nuclear neurons. For this

reason, the airpuff-US produces a strong hyperpolarization on the postsynaptic AIN neuron, which is followed by a rebound burst of action potentials. A series of airpuff-US presentations (hyperpolarization followed by rebound depolarization) appears to be sufficient to elicit an increase in membrane excitability on eyeblink AIN neurons (Aizenman and Linden, 2000; Zhang et al., 2004; Molineux et al., 2008; Tadayonnejad et al., 2010).

This process is mediated by $\mathrm{Ca} 2+$ signals leading to the insertion of new voltage-gated ion channels. Even more, an increase in membrane excitability appears to confine the neuron to a permissive state that is necessary for the induction of synaptic plasticity during learning (Schreurs et al., 1991; Schreurs et al., 1997; Schreurs et al., 1998; Wang and Schreurs, 2006; Belmeguenai et al., 2010; Mozzachiodi and Byrne, 2010; Wang and Schreurs, 2010). 


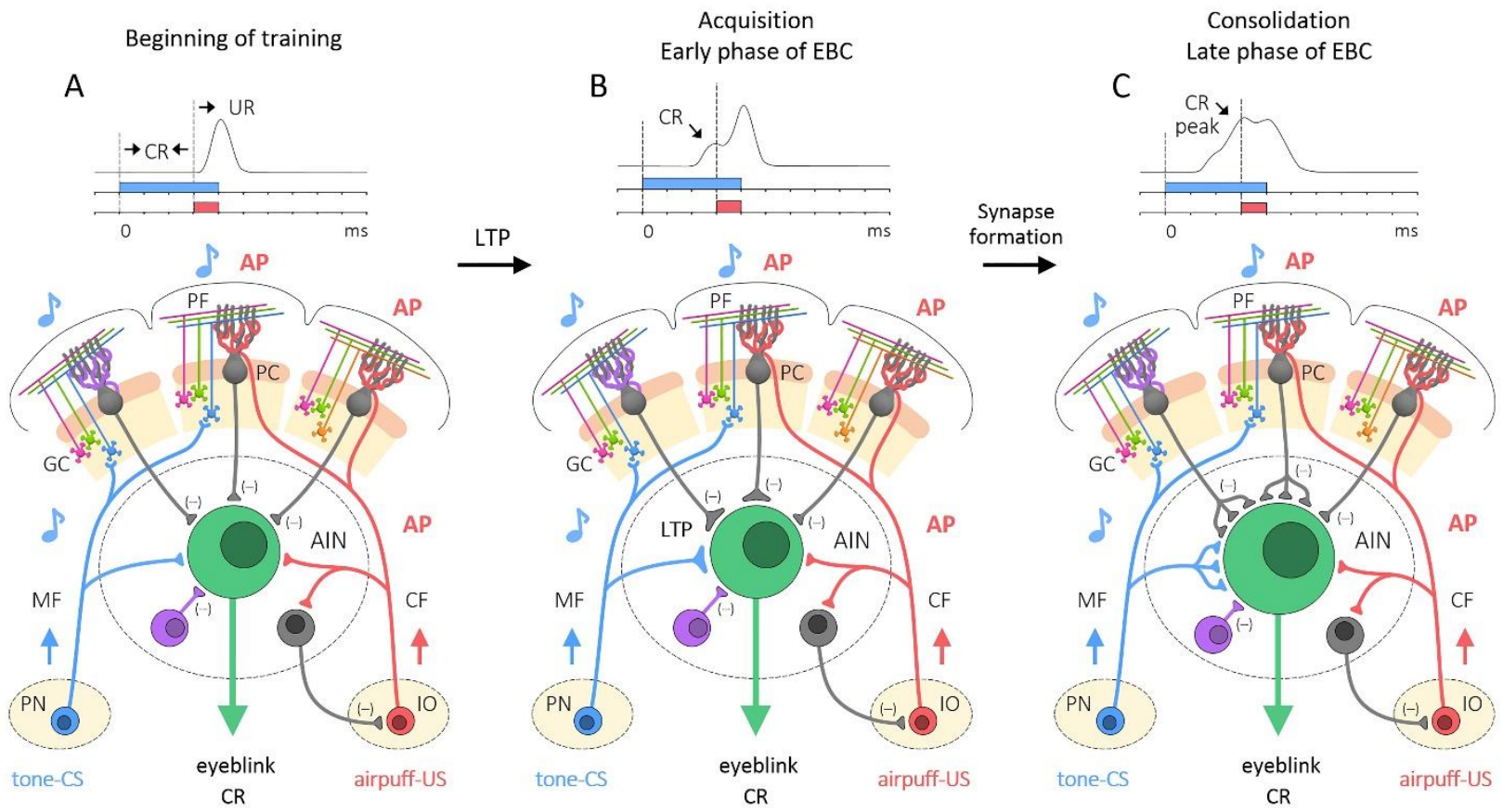

Figure 32. Synaptic mechanisms underlying learning in eyeblink conditioning. $\mathrm{A}$, the beginning of paired delay training during eyeblink conditioning $(\mathrm{EBC})$. $\mathrm{B}$, the early phase of EBC during the acquisition of the first CRs. C, the late phase of EBC during the consolidation of CRs.

After increased excitability, the second step of learning takes place as an association between the stimuli is formed and the first CRs emerge (Figure 32 B). An increase in membrane excitability facilitates the subsequent potentiation of MF and PC synapses that convey information about the tone-CS to the postsynaptic eyeblink AIN neurons. In our proposed model of learning, potentiation of both, MF and PC synapses that are activated by the tone-CS is necessary for the formation of the first CRs. 
Potentiation of MF and PC synapses is regulated by PC that are activated by the airpuff-US. Selective potentiation of specific MF synapses is mediated by a two-step process: a priming step that is followed by a potentiation step. The tone-CS activates NMDARs in specific MF synapses. Ca2+ influx through NMDARs serves as a tagging mechanism that primes recently activated MF synapses. The hyperpolarization-rebound elicited by the airpuff-US triggers the potentiation of recently activated and primed MF synapses.

On the other hand, potentiation of tone-activated PC synapses is mediated by airpuff-activated PCs. The airpuff-US activates many eyeblink PCs that in turn produce a strong hyperpolarization on the postsynaptic neuron that is followed by a rebound burst of action potentials. In short, PC synapses that are active before the period that successfully triggers a rebound burst of action potentials on the postsynaptic nuclear neuron undergo LTP (Morishita and Sastry, 1993; Sastry et al., 1997; Aizenman et al., 2000; Ouardouz and Sastry, 2000). This type of LTP is also Ca2+ dependent and the number of action potentials elicited after the rebound greatly influences the magnitude of the potentiation (Sastry et al., 1997; Aizenman et al., 2000).

In this manner, a rebound burst generated by the airpuff-activated PCs triggers LTP at the weaker tone-activated PC synapses. Potentiation is mediated by increasing the number of receptors in the PSD that leads to an increase in the size and efficacy of tone-activated MF and PC synapses. The increase in synaptic size and efficacy allows the tone-CS to activate eyeblink AIN neurons and trigger the first eyeblink CRs. Consequently, repeated tone-CS and airpuff-US during paired training produce a simultaneous and parallel increase in MF and PC synapses that together may provide the precise sequence of signals to produce the necessary response on the postsynaptic eyeblink AIN to convey timely and meaningful commands to the RN.

The third and last step of learning involves synaptogenesis of previously potentiated synapses as CRs are consolidated during the late phase of EBC (Figure 32 C). This process is mediated by the division of previously potentiated synapses and the formation of new ones. Moreover, learning in the AIN requires complementary synaptic plasticity in the cerebellar cortex as PCs shift and synchronize their response to occur shortly after the tone onset that is followed by a pause. 
Consequently, plasticity in the cerebellar cortex allows PCs to deliver a brief and synchronized burst of inhibitory action potentials immediately after the tone onset and produce a large hyperpolarization on the postsynaptic neuron that is followed by a rebound burst. In this manner eyeblink projection neurons can execute precise CRs in response to the tone-CS when MF excitation is immediately followed by a brief and synchronized burst of PC inputs. Together this evidence supports a learning mechanism in the cerebellum that is mediated by complementary changes in the cerebellar cortex and AIN that allow predictive signals to coincide in eyeblink projection neurons and elicit a precisely timed CR. 


\section{REFERENCES}

Aizenman CD, Linden DJ (1999) Regulation of the rebound depolarization and spontaneous firing patterns of deep nuclear neurons in slices of rat cerebellum. J Neurophysiol 82:1697-1709.

Aizenman CD, Linden DJ (2000) Rapid, synaptically driven increases in the intrinsic excitability of cerebellar deep nuclear neurons. Nat Neurosci 3:109-111.

Aizenman CD, Manis PB, Linden DJ (1998) Polarity of long-term synaptic gain change is related to postsynaptic spike firing at a cerebellar inhibitory synapse. Neuron 21:827-835.

Aizenman CD, Huang EJ, Linden DJ (2003) Morphological correlates of intrinsic electrical excitability in neurons of the deep cerebellar nuclei. J Neurophysiol 89:1738-1747.

Aizenman CD, Huang EJ, Manis PB, Linden DJ (2000) Use-dependent changes in synaptic strength at the Purkinje cell to deep nuclear synapse. Prog Brain Res 124:257-273.

Akaike T (1992) The tectorecipient zone in the inferior olivary nucleus in the rat. J Comp Neurol 320:398-414.

Albus JS (1971) A theory of cerebellar function. Math Biosci 10:25-61.

Allen MT, Chelius L, Masand V, Gluck MA, Myers CE, Schnirman G (2002) A comparison of latent inhibition and learned irrelevance pre-exposure effects in rabbit and human eyeblink conditioning. Integr Physiol Behav Sci 37:188-214.

Alvina K, Walter JT, Kohn A, Ellis-Davies G, Khodakhah K (2008) Questioning the role of rebound firing in the cerebellum. Nat Neurosci 11:1256-1258.

Anchisi D, Scelfo B, Tempia F (2001) Postsynaptic currents in deep cerebellar nuclei. J Neurophysiol 85:323-331.

Andersson G, Garwicz M, Hesslow G (1988) Evidence for a GABA-mediated cerebellar inhibition of the inferior olive in the cat. Exp Brain Res 72:450-456.

Andlauer TF, Sigrist SJ (2012) Quantitative analysis of Drosophila larval neuromuscular junction morphology. Cold Spring Harb Protoc 2012:490-493.

Angaut P, Sotelo C (1973) The fine structure of the cerebellar central nuclei in the cat. II. Synaptic organization. Exp Brain Res 16:431-454.

Apps R (1999) Movement-related gating of climbing fibre input to cerebellar cortical zones. Prog Neurobiol 57:537-562.

Apps R, Hawkes R (2009) Cerebellar cortical organization: a one-map hypothesis. Nat Rev Neurosci 10:670-681. 
Arellano JI, Benavides-Piccione R, Defelipe J, Yuste R (2007a) Ultrastructure of dendritic spines: correlation between synaptic and spine morphologies. Front Neurosci 1:131-143.

Arellano JI, Benavides-Piccione R, Defelipe J, Yuste R (2007b) Ultrastructure of dendritic spines: correlation between synaptic and spine morphologies. Front Neurosci 1:131-143.

Armstrong DM (1974) Functional significance of connections of the inferior olive. Physiol Rev 54:358417.

Armstrong DM, Schild RF (1978) An investigation of the cerebellar corticonuclear projections in the rat using an autoradiographic tracing method. II. Projections from the hemisphere. Brain Res 141:235-249.

Aston-Jones G, Card JP (2000) Use of pseudorabies virus to delineate multisynaptic circuits in brain: opportunities and limitations. J Neurosci Methods 103:51-61.

Aston-Jones G, Zhu Y, Card JP (2004) Numerous GABAergic afferents to locus ceruleus in the pericerulear dendritic zone: possible interneuronal pool. J Neurosci 24:2313-2321.

Attwell PJ, Rahman S, Yeo CH (2001) Acquisition of eyeblink conditioning is critically dependent on normal function in cerebellar cortical lobule HVI. J Neurosci 21:5715-5722.

Avendano C, Machin R, Bermejo PE, Lagares A (2005) Neuron numbers in the sensory trigeminal nuclei of the rat: A GABA- and glycine-immunocytochemical and stereological analysis. J Comp Neurol 493:538-553.

Bagnall MW, Zingg B, Sakatos A, Moghadam SH, Zeilhofer HU, du Lac S (2009) Glycinergic projection neurons of the cerebellum. J Neurosci 29:10104-10110.

Bartha A (1961) Experimental reduction of virulence of Aujesky's disease. 16:42-45.

Batini C, Billard JM (1985) Release of cerebellar inhibition by climbing fiber deafferentation. Exp Brain Res 57:370-380.

Batini C, Compoint C, Buisseret-Delmas C, Daniel H, Guegan M (1992) Cerebellar nuclei and the nucleocortical projections in the rat: retrograde tracing coupled to GABA and glutamate immunohistochemistry. J Comp Neurol 315:74-84.

Baurle J, Grusser-Cornehls U (1997) Differential number of glycine- and GABA-immunopositive neurons and terminals in the deep cerebellar nuclei of normal and Purkinje cell degeneration mutant mice. J Comp Neurol 382:443-458.

Belmeguenai A, Hosy E, Bengtsson F, Pedroarena CM, Piochon C, Teuling E, He Q, Ohtsuki G, De Jeu MT, Elgersma Y, De Zeeuw CI, Jorntell H, Hansel C (2010) Intrinsic plasticity complements long-term potentiation in parallel fiber input gain control in cerebellar Purkinje cells. J Neurosci 30:13630-13643.

Benarroch EE (2011) Glycine and its synaptic interactions: functional and clinical implications. Neurology 77:677-683.

Benedetti F, Montarolo PG, Strata P, Tempia F (1983) Inferior olive inactivation decreases the excitability of the intracerebellar and lateral vestibular nuclei in the rat. J Physiol 340:195-208. 
Bengtsson F, Ekerot CF, Jorntell H (2011) In vivo analysis of inhibitory synaptic inputs and rebounds in deep cerebellar nuclear neurons. PLoS One 6:e18822.

Bennett CH, Maldonado A, Mackintosh NJ (1995) Learned irrelevance is not the sum of exposure to CS and US. Q J Exp Psychol B 48:117-128.

Berkley KJ, Hand PJ (1978) Projections to the inferior olive of the cat. II. Comparisons of input from the gracile, cuneate and the spinal trigeminal nuclei. J Comp Neurol 180:253-264.

Berthier NE (1984) The role of the extraocular muscles in the rabbit nictitating membrane response: a reexamination. Behav Brain Res 14:81-84.

Berthier NE (1992) Muscle activity during unconditioned and conditioned eye blinks in the rabbit. Behav Brain Res 48:21-28.

Berthier NE, Moore JW (1983) The nictitating membrane response: an electrophysiological study of the abducens nerve and nucleus and the accessory abducens nucleus in rabbit. Brain Res 258:201-210.

Berthier NE, Moore JW (1986) Cerebellar Purkinje cell activity related to the classically conditioned nictitating membrane response. Exp Brain Res 63:341-350.

Berthier NE, Moore JW (1990) Activity of deep cerebellar nuclear cells during classical conditioning of nictitating membrane extension in rabbits. Exp Brain Res 83:44-54.

Billig I, Card JP, Yates BJ (2003) Neurochemical phenotypes of MRF neurons influencing diaphragm and rectus abdominis activity. J Appl Physiol (1985) 94:391-398.

Billig I, Foris JM, Enquist LW, Card JP, Yates BJ (2000) Definition of neuronal circuitry controlling the activity of phrenic and abdominal motoneurons in the ferret using recombinant strains of pseudorabies virus. J Neurosci 20:7446-7454.

Blenkinsop TA, Lang EJ (2011) Synaptic action of the olivocerebellar system on cerebellar nuclear spike activity. J Neurosci 31:14708-14720.

Boele HJ, Koekkoek SK, De Zeeuw CI, Ruigrok TJ (2013) Axonal sprouting and formation of terminals in the adult cerebellum during associative motor learning. J Neurosci 33:17897-17907.

Boesten AJ, Voogd J (1975) Projections of the dorsal column nuclei and the spinal cord on the inferior olive in the cat. J Comp Neurol 161:215-237.

Bonis JM, Neumueller SE, Krause KL, Kiner T, Smith A, Marshall BD, Qian B, Pan LG, Forster HV (2010) The pontine respiratory group, particularly the Kolliker-Fuse nucleus, mediates phases of the hypoxic ventilatory response in unanesthetized goats. J Appl Physiol (1985) 108:1321-1335.

Booth ES (1948) Laboratory anatomy of the cat. Dubuque, Iowa: W. C. Brown.

Boyce RW, Dorph-Petersen KA, Lyck L, Gundersen HJ (2010) Design-based stereology: introduction to basic concepts and practical approaches for estimation of cell number. Toxicol Pathol 38:1011-1025. 
Bracha V, Irwin KB, Webster ML, Wunderlich DA, Stachowiak MK, Bloedel JR (1998) Microinjections of anisomycin into the intermediate cerebellum during learning affect the acquisition of classically conditioned responses in the rabbit. Brain Res 788:169-178.

Branco T, Marra V, Staras K (2010) Examining size-strength relationships at hippocampal synapses using an ultrastructural measurement of synaptic release probability. J Struct Biol 172:203-210.

Brittle EE, Reynolds AE, Enquist LW (2004) Two modes of pseudorabies virus neuroinvasion and lethality in mice. J Virol 78:12951-12963.

Brochu G, Maler L, Hawkes R (1990) Zebrin II: a polypeptide antigen expressed selectively by Purkinje cells reveals compartments in rat and fish cerebellum. J Comp Neurol 291:538-552.

Brodal A, Jansen J (1946) The ponto-cerebellar projection in the rabbit and cat; experimental investigations. J Comp Neurol 84:31-118.

Brodal A, Walberg F, Hoddevik GH (1975) The olivocerebellar projection in the cat studied with the method of retrograde axonal transport of horseradish peroxidase. J Comp Neurol 164:449-469.

Brooks JX, Cullen KE (2009) Multimodal integration in rostral fastigial nucleus provides an estimate of body movement. J Neurosci 29:10499-10511.

Brown JT, Chan-Palay V, Palay SL (1977) A study of afferent input to the inferior olivary complex in the rat by retrograde axonal transport of horseradish peroxidase. J Comp Neurol 176:1-22.

Burli T, Baer K, Ewers H, Sidler C, Fuhrer C, Fritschy JM (2010) Single particle tracking of alpha7 nicotinic $\mathrm{AChR}$ in hippocampal neurons reveals regulated confinement at glutamatergic and GABAergic perisynaptic sites. PLoS One 5:e11507.

Burrone J, Murthy VN (2001) Synaptic plasticity: rush hour traffic in the AMPA lanes. Curr Biol 11:R274-277.

Buttner U, Fuchs AF, Markert-Schwab G, Buckmaster P (1991) Fastigial nucleus activity in the alert monkey during slow eye and head movements. J Neurophysiol 65:1360-1371.

Calhoun ME, Jucker M, Martin LJ, Thinakaran G, Price DL, Mouton PR (1996) Comparative evaluation of synaptophysin-based methods for quantification of synapses. J Neurocytol 25:821-828.

Campbell NC, Hesslow G (1986a) The secondary spikes of climbing fibre responses recorded from Purkinje cell somata in cat cerebellum. J Physiol 377:207-224.

Campbell NC, Hesslow G (1986b) The secondary spikes of climbing fibre responses recorded from Purkinje cell axons in cat cerebellum. J Physiol 377:225-235.

Campolattaro MM, Kashef A, Lee I, Freeman JH (2011) Neuronal correlates of cross-modal transfer in the cerebellum and pontine nuclei. J Neurosci 31:4051-4062.

Card JP (1998) Practical considerations for the use of pseudorabies virus in transneuronal studies of neural circuitry. Neurosci Biobehav Rev 22:685-694. 
Cegavske CF, Thompson RF (1976) Mechanisms of efferent neuronal control of the reflex nicitating membrane response in rabbit (Oryctolagus cuniculus). J Comp Physiol Psychol 90:411-423.

Cegavske CF, Patterson MM, Thompson RF (1979) Neuronal unit activity in the abducens nucleus during classical conditioning of the nictitating membrane response in the rabbit (Oryctolagus cuniculus). J Comp Physiol Psychol 93:595-609.

Cerminara NL, Apps R (2011) Behavioural significance of cerebellar modules. Cerebellum (London, England) 10:484-494.

Chagnaud JL, Campistron G, Geffard M (1989) Monoclonal antibody directed against glutaraldehyde conjugated glutamate and immunocytochemical applications in the rat brain. Brain Res 481:175-180.

Chalazonitis A, Pham TD, Li Z, Roman D, Guha U, Gomes W, Kan L, Kessler JA, Gershon MD (2008) Bone morphogenetic protein regulation of enteric neuronal phenotypic diversity: relationship to timing of cell cycle exit. J Comp Neurol 509:474-492.

Chamberlin NL, Saper CB (1994) Topographic organization of respiratory responses to glutamate microstimulation of the parabrachial nucleus in the rat. J Neurosci 14:6500-6510.

Chan-Palay V (1971) The recurrent collaterals of Purkinje cell axons: a correlated study of the rat's cerebellar cortex with electron microscopy and the Golgi method. In, pp 200-234.

Chan-Palay V (1973a) The cytology of neurons and their dendrites in the simple mammalian nucleus lateralis. An electron microscope study. In, pp 289-317.

Chan-Palay V (1973b) Axon terminals of the intrinsic neurons in the nucleus lateralis of the cerebellum. An electron microscope study. In, pp 187-206.

Chan-Palay V (1973c) On the identification of the afferent axon terminals in the nucleus lateralis of the cerebellum. An electron microscope study. Z Anat Entwicklungsgesch 142:149-186.

Chan-Palay V (1973d) Afferent axons and their relations with neurons in the nucleus lateralis of the cerebellum: a light microscopic study. In, pp 1-21.

Chan-Palay V (1973e) Cytology and organization in the nucleus lateralis of the cerebellum: the projections of neurons and their processes into afferent axon bundles. In, pp 151-159.

Chapman PF, Steinmetz JE, Thompson RF (1988) Classical conditioning does not occur when direct stimulation of the red nucleus or cerebellar nuclei is the unconditioned stimulus. Brain Res 442:97-9104.

Chemin K, Bohineust A, Dogniaux S, Tourret M, Guegan S, Miro F, Hivroz C (2012) Cytokine secretion by CD4+ T cells at the immunological synapse requires Cdc42-dependent local actin remodeling but not microtubule organizing center polarity. J Immunol 189:2159-2168.

Chen G, Steinmetz JE (2000) Intra-cerebellar infusion of NMDA receptor antagonist AP5 disrupts classical eyeblink conditioning in rabbits. Brain Res 887:144-156.

Chen S, Hillman DE (1993) Colocalization of neurotransmitters in the deep cerebellar nuclei. J Neurocytol 22:81-91. 
Chen S, Yang M, Miselis RR, Aston-Jones G (1999) Characterization of transsynaptic tracing with central application of pseudorabies virus. Brain Res 838:171-183.

Chien CH, Shieh JY, Liao MH, Ling EA, Wen CY (1998) Neuronal connections between the auricular skin and the sympathetic pre- and postganglionic neurons of the dog as studied by using pseudorabies virus. Neurosci Res 30:169-175.

Clark GA, McCormick DA, Lavond DG, Thompson RF (1984) Effects of lesions of cerebellar nuclei on conditioned behavioral and hippocampal neuronal responses. Brain Res 291:125-136.

Clements JR, Magnusson KR, Beitz AJ (1990) Ultrastructural description of glutamate-, aspartate-, taurine-, and glycine-like immunoreactive terminals from five rat brain regions. J Electron Microsc Tech 15:49-66.

Crook J, Hendrickson A, Robinson FR (2006) Co-localization of glycine and gaba immunoreactivity in interneurons in Macaca monkey cerebellar cortex. Neuroscience 141:1951-1959.

Curanovic D, Enquist L (2009) Directional transneuronal spread of alpha-herpesvirus infection. Future Virol 4:591.

Curanovic D, Lyman MG, Bou-Abboud C, Card JP, Enquist LW (2009) Repair of the UL21 locus in pseudorabies virus Bartha enhances the kinetics of retrograde, transneuronal infection in vitro and in vivo. J Virol 83:1173-1183.

Czubayko U, Sultan F, Thier P, Schwarz C (2001) Two types of neurons in the rat cerebellar nuclei as distinguished by membrane potentials and intracellular fillings. J Neurophysiol 85:2017-2029.

Daniel H, Billard JM, Angaut P, Batini C (1987) The interposito-rubrospinal system. Anatomical tracing of a motor control pathway in the rat. Neurosci Res 5:87-112.

Daniels D, Miselis RR, Flanagan-Cato LM (1999) Central neuronal circuit innervating the lordosisproducing muscles defined by transneuronal transport of pseudorabies virus. J Neurosci 19:2823-2833.

de Groat WC, Araki I, Vizzard MA, Yoshiyama M, Yoshimura N, Sugaya K, Tai C, Roppolo JR (1998) Developmental and injury induced plasticity in the micturition reflex pathway. Behav Brain Res 92:127140.

De Zeeuw CI, Berrebi AS (1995) Postsynaptic targets of Purkinje cell terminals in the cerebellar and vestibular nuclei of the rat. Eur J Neurosci 7:2322-2333.

De Zeeuw CI, Holstege JC, Ruigrok TJ, Voogd J (1989) Ultrastructural study of the GABAergic, cerebellar, and mesodiencephalic innervation of the cat medial accessory olive: anterograde tracing combined with immunocytochemistry. J Comp Neurol 284:12-35.

Deaux EB, Gormezano I (1963) Eyeball retraction: classical conditioning and extinction in the albino rabbit. Science 141:630-631.

Delgado-Garcia JM, Gruart A, Trigo JA (2003) Physiology of the eyelid motor system. Ann N Y Acad Sci 1004:1-9. 
Delhaye-Bouchaud N, Geoffroy B, Mariani J (1985) Neuronal death and synapse elimination in the olivocerebellar system. I. Cell counts in the inferior olive of developing rats. J Comp Neurol 232:299308.

Desmond JE, Moore JW (1991) Single-unit activity in red nucleus during the classically conditioned rabbit nictitating membrane response. Neurosci Res 10:260-279.

Disterhoft JF, Quinn KJ, Weiss C, Shipley MT (1985) Accessory abducens nucleus and conditioned eye retraction/nictitating membrane extension in rabbit. J Neurosci 5:941-950.

Dmitrieva NA, Lindstrom JM, Keyser KT (2001) The relationship between GABA-containing cells and the cholinergic circuitry in the rabbit retina. Vis Neurosci 18:93-9100.

Downie LE, Hatzopoulos KM, Pianta MJ, Vingrys AJ, Wilkinson-Berka JL, Kalloniatis M, Fletcher EL (2010) Angiotensin type-1 receptor inhibition is neuroprotective to amacrine cells in a rat model of retinopathy of prematurity. J Comp Neurol 518:41-63.

Dresbach T, Nawrotzki R, Kremer T, Schumacher S, Quinones D, Kluska M, Kuhse J, Kirsch J (2008) Molecular architecture of glycinergic synapses. Histochem Cell Biol 130:617-633.

Dufour A, Tell F, Kessler JP, Baude A (2010) Mixed GABA-glycine synapses delineate a specific topography in the nucleus tractus solitarii of adult rat. J Physiol 588:1097-1115.

Dugue GP, Dumoulin A, Triller A, Dieudonne S (2005) Target-dependent use of co-released inhibitory transmitters at central synapses. J Neurosci 25:6490-6498.

Dumoulin A, Triller A, Dieudonne S (2001) IPSC kinetics at identified GABAergic and mixed GABAergic and glycinergic synapses onto cerebellar Golgi cells. J Neurosci 21:6045-6057.

Eager RP (1968) Some fine structural features of the neural elements composing the cerebellar nuclei in the cat. J Comp Neurol 132:235-262.

Eccles JC (1973) The cerebellum as a computer: patterns in space and time. J Physiol 229:1-32.

Eccles JC, Scheid P, Taborikova H (1975) Responses of red nucleus neurons to antidromic and synaptic activation. J Neurophysiol 38:947-964.

Eccles JCI, M.; Szentágothai, J. (1967) The cerebellum as a neuronal machine. Berlin: Springer-Verlag.

Ekerot CF, Larson B (1979) The dorsal spino-olivocerebellar system in the cat. II. Somatotopical organization. Exp Brain Res 36:219-232.

El Majdoubi M, Poulain DA, Theodosis DT (1997) Lactation-induced plasticity in the supraoptic nucleus augments axodendritic and axosomatic GABAergic and glutamatergic synapses: an ultrastructural analysis using the disector method. Neuroscience 80:1137-1147.

Enger M, Brodal P (1985) Organization of corticopontocerebellar connections to the paramedian lobule in the cat. Anat Embryol (Berl) 172:227-238. 
Enquist LW (1999) Life beyond eradication: veterinary viruses in basic science. Arch Virol Suppl 15:87109.

Fanardjian VV, Sarkissian JS, Manvelian IA (1987) Synaptic responses of red nucleus neurons in the alert cat to cortical and cerebellar inputs. Neurosci Lett 73:38-42.

Fay Ra, Norgren R (1997) Identification of rat brainstem multisynaptic connections to the oral motor nuclei in the rat using pseudorabies virus. II. Facial muscle motor systems. Brain research Brain research reviews 25:276-290.

Flumerfelt BA (1978) Organization of the mammalian red nucleus and its interconnections with the cerebellum. Experientia 34:1178-1179.

Freas CA, Bingman K, Ladage LD, Pravosudov VV (2013) Untangling elevation-related differences in the hippocampus in food-caching mountain chickadees: the effect of a uniform captive environment. Brain Behav Evol 82:199-209.

Fredrich M, Reisch A, Illing R-B (2009) Neuronal subtype identity in the rat auditory brainstem as defined by molecular profile and axonal projection. Exp Brain Res 195:241-260.

Freeman JH, Jr., Nicholson DA (2000) Developmental changes in eye-blink conditioning and neuronal activity in the cerebellar interpositus nucleus. J Neurosci 20:813-819.

Fritschy J-M, Harvey RJ, Schwarz G (2008) Gephyrin: where do we stand, where do we go? Trends Neurosci 31:257-264.

Fukazawa Y, Tarusawa E, Matsui K, Shigemoto R (2008) [Ultrastructural insights of postsynaptic glutamate receptor organization]. Tanpakushitsu Kakusan Koso 53:436-441.

Furutani R, Sugita S (2008) Comparative histological study of the mammalian facial nucleus. J Vet Med Sci 70:367-372.

Garcia-Junco-Clemente P, Cantero G, Gomez-Sanchez L, Linares-Clemente P, Martinez-Lopez JA, Lujan R, Fernandez-Chacon R (2010) Cysteine string protein-alpha prevents activity-dependent degeneration in GABAergic synapses. J Neurosci 30:7377-7391.

Garcia KS, Steele PM, Mauk MD (1999) Cerebellar cortex lesions prevent acquisition of conditioned eyelid responses. J Neurosci 19:10940-10947.

Gardner EP, Fuchs AF (1975) Single-unit responses to natural vestibular stimuli and eye movements in deep cerebellar nuclei of the alert rhesus monkey. J Neurophysiol 38:627-649.

Gibson AR, Houk JC, Kohlerman NJ (1985) Relation between red nucleus discharge and movement parameters in trained macaque monkeys. J Physiol 358:551-570.

Gonzalez-Joekes J, Schreurs BG (2012) Anatomical characterization of a rabbit cerebellar eyeblink premotor pathway using pseudorabies and identification of a local modulatory network in anterior interpositus. J Neurosci 32:12472-12487. 
Gormezano I, Schneiderman N, Deaux E, Fuentes I (1962) Nictitating membrane: classical conditioning and extinction in the albino rabbit. Science 138:33-34.

Gould TJ, Steinmetz JE (1996) Changes in rabbit cerebellar cortical and interpositus nucleus activity during acquisition, extinction, and backward classical eyelid conditioning. Neurobiol Learn Mem 65:1734.

Grant K, Horcholle-Bossavit G (1986) Red nucleus inputs to retractor bulbi motoneurones in the cat. J Physiol 371:317-327.

Gray EG (1959) Axo-somatic and axo-dendritic synapses of the cerebral cortex: an electron microscope study. J Anat 93:420-433.

Gray EG (1969) Electron microscopy of excitatory and inhibitory synapses: a brief review. Prog Brain Res 31:141-155.

Gray TS, McMaster SE, Harvey JA, Gormezano I (1981) Localization of retractor bulbi motoneurons in the rabbit. Brain Res 226:93-106.

Green JT, Steinmetz JE (2005) Purkinje cell activity in the cerebellar anterior lobe after rabbit eyeblink conditioning. Learn Mem 12:260-269.

Gruart A, Schreurs BG, del Toro ED, Delgado-Garcia JM (2000) Kinetic and frequency-domain properties of reflex and conditioned eyelid responses in the rabbit. J Neurophysiol 83:836-852.

Haley DA, Thompson RF, Madden Jt (1988) Pharmacological analysis of the magnocellular red nucleus during classical conditioning of the rabbit nictitating membrane response. Brain Res 454:131-139.

Halverson HE, Hubbard EM, Freeman JH (2009) Stimulation of the lateral geniculate, superior colliculus, or visual cortex is sufficient for eyeblink conditioning in rats. Learn Mem 16:300-307.

Harvey JA, Land T, McMaster SE (1984) Anatomical study of the rabbit's corneal-VIth nerve reflex: connections between cornea, trigeminal sensory complex, and the abducens and accessory abducens nuclei. Brain Res 301:307-321.

Henriquez VM, Evinger C (2007) The three-neuron corneal reflex circuit and modulation of second-order corneal responsive neurons. Exp Brain Res 179:691-702.

Hesslow G (1994a) Inhibition of classically conditioned eyeblink responses by stimulation of the cerebellar cortex in the decerebrate cat. J Physiol 476:245-256.

Hesslow G (1994b) Correspondence between climbing fibre input and motor output in eyeblink-related areas in cat cerebellar cortex. The Journal of physiology 476:229-244.

Hesslow G, Jirenhed DA, Rasmussen A, Johansson F (2013) Classical conditioning of motor responses: what is the learning mechanism? Neural Netw 47:81-87.

Hinrichsen CF, Watson CD (1983) Brain stem projections to the facial nucleus of the rat. Brain Behav Evol 22:153-163. 
Hiraoka M, Shimamura M (1977) Neural mechanisms of the corneal blinking reflex in cats. Brain Res 125:265-275.

Hoebeek FE, Khosrovani S, Witter L, De Zeeuw CI (2008) Purkinje cell input to cerebellar nuclei in tottering: ultrastructure and physiology. Cerebellum (London, England) 7:547-558.

Hoebeek FE, Witter L, Ruigrok TJ, De Zeeuw CI (2010) Differential olivo-cerebellar cortical control of rebound activity in the cerebellar nuclei. Proc Natl Acad Sci U S A 107:8410-8415.

Holstege G, Tan J (1988) Projections from the red nucleus and surrounding areas to the brainstem and spinal cord in the cat. An HRP and autoradiographical tracing study. Behav Brain Res 28:33-57.

Horta-Junior JAC, Tamega OJ, Cruz-Rizzolo RJ (2004) Cytoarchitecture and musculotopic organization of the facial motor nucleus in Cebus apella monkey. J Anat 204:175-190.

Ikeda M, Matsushita M (1973) Electron microscopic observations on the spinal projections to the cerebellar nuclei in the cat and rabbit. Experientia 29:1280-1282.

Ilha J, Centenaro LA, Broetto Cunha N, de Souza DF, Jaeger M, do Nascimento PS, Kolling J, Ben J, Marcuzzo S, Wyse AT, Gottfried C, Achaval M (2011) The beneficial effects of treadmill step training on activity-dependent synaptic and cellular plasticity markers after complete spinal cord injury. Neurochem Res 36:1046-1055.

Isope P, Barbour B (2002) Properties of unitary granule cell-->Purkinje cell synapses in adult rat cerebellar slices. J Neurosci 22:9668-9678.

Ito M (2006) Cerebellar circuitry as a neuronal machine. Prog Neurobiol 78:272-303.

Ito M, Yoshida M, Obata K (1964) Monosynaptic inhibition of the intracerebellar nuclei induced rom the cerebellar cortex. Experientia 20:575-576.

Ito M, Sakurai M, Tongroach P (1982) Climbing fibre induced depression of both mossy fibre responsiveness and glutamate sensitivity of cerebellar Purkinje cells. J Physiol 324:113-134.

Ito S, Takeichi M (2009) Dendrites of cerebellar granule cells correctly recognize their target axons for synaptogenesis in vitro. Proc Natl Acad Sci U S A 106:12782-12787.

James P, Rivier C, Lee S (2008) Presence of corticotrophin-releasing factor and/or tyrosine hydroxylase in cells of a neural brain-testicular pathway that are labelled by a transganglionic tracer. $\mathrm{J}$

Neuroendocrinol 20:173-181.

Jirenhed DA, Bengtsson F, Hesslow G (2007) Acquisition, extinction, and reacquisition of a cerebellar cortical memory trace. J Neurosci 27:2493-2502.

Johnson JW, Ascher P (1987) Glycine potentiates the NMDA response in cultured mouse brain neurons. Nature 325:529-531.

Johnson RD, Chadha HK, Dugan VP, Gupta DS, Ferrero SL, Hubscher CH (2011) Bilateral bulbospinal projections to pudendal motoneuron circuitry after chronic spinal cord hemisection injury as revealed by transsynaptic tracing with pseudorabies virus. J Neurotrauma 28:595-605. 
Jovanovic K, Pastor AM, O'Donovan MJ (2010) The use of PRV-Bartha to define premotor inputs to lumbar motoneurons in the neonatal spinal cord of the mouse. PLoS One 5:e11743.

Kalmbach BE, Davis T, Ohyama T, Riusech F, Nores WL, Mauk MD (2010) Cerebellar cortex contributions to the expression and timing of conditioned eyelid responses. J Neurophysiol 103:20392049.

Kellett DO, Fukunaga I, Chen-Kubota E, Dean P, Yeo CH (2010) Memory consolidation in the cerebellar cortex. PLoS One 5:e11737.

Kharazia VN, Weinberg RJ (1999) Immunogold localization of AMPA and NMDA receptors in somatic sensory cortex of albino rat. J Comp Neurol 412:292-302.

Kharazia VN, Phend KD, Rustioni A, Weinberg RJ (1996) EM colocalization of AMPA and NMDA receptor subunits at synapses in rat cerebral cortex. Neurosci Lett 210:37-40.

Kim JJ, Thompson RF (1997) Cerebellar circuits and synaptic mechanisms involved in classical eyeblink conditioning. Trends Neurosci 20:177-181.

Kleim JA, Freeman JH, Bruneau R, Nolan BC, Cooper NR, Zook A, Walters D (2002) Synapse formation is associated with memory storage in the cerebellum. Proc Natl Acad Sci U S A 99:13228-13231.

Klein BG, Rhoades RW, Jacquin MF (1990) Topography of the facial musculature within the facial (VII) motor nucleus of the neonatal rat. Exp Brain Res 81:649-653.

Klemann CJ, Roubos EW (2011) The gray area between synapse structure and function-Gray's synapse types I and II revisited. Synapse 65:1222-1230.

Komiyama M, Shibata H, Suzuki T (1984) Somatotopic representation of facial muscles within the facial nucleus of the mouse. A study using the retrograde horseradish peroxidase and cell degeneration techniques. Brain Behav Evol 24:144-151.

Kotani S, Kawahara S, Kirino Y (2003) Purkinje cell activity during learning a new timing in classical eyeblink conditioning. Brain Res 994:193-202.

Krupa DJ, Thompson RF (1995) Inactivation of the superior cerebellar peduncle blocks expression but not acquisition of the rabbit's classically conditioned eye-blink response. Proc Natl Acad Sci U S A 92:5097-5101.

Krupa DJ, Thompson RF (1997) Reversible inactivation of the cerebellar interpositus nucleus completely prevents acquisition of the classically conditioned eye-blink response. Learn Mem 3:545-556.

Krupa DJ, Thompson JK, Thompson RF (1993) Localization of a memory trace in the mammalian brain. Science 260:989-991.

Kubota Y, Kawaguchi Y (2000) Dependence of GABAergic synaptic areas on the interneuron type and target size. J Neurosci 20:375-386.

Kuo SP, Bradley LA, Trussell LO (2009) Heterogeneous kinetics and pharmacology of synaptic inhibition in the chick auditory brainstem. J Neurosci 29:9625-9634. 
Lashley KS, Wade M (1946) The Pavlovian theory of generalization. Psychol Rev 53:72-87.

Lavond DG, Lincoln JS, McCormick DA, Thompson RF (1984) Effect of bilateral lesions of the dentate and interpositus cerebellar nuclei on conditioning of heart-rate and nictitating membrane/eyelid responses in the rabbit. Brain Res 305:323-330.

Lavond DG, Knowlton BJ, Steinmetz JE, Thompson RF (1987) Classical conditioning of the rabbit eyelid response with a mossy-fiber stimulation CS: II. Lateral reticular nucleus stimulation. Behav Neurosci 101:676-682.

Leal-Campanario R, Barradas-Bribiescas JA, Delgado-Garcia JM, Gruart A (2004a) Relative contributions of eyelid and eye-retraction motor systems to reflex and classically conditioned blink responses in the rabbit. J Appl Physiol 96:1541-1554.

Leal-Campanario R, Barradas-Bribiescas JA, Delgado-Garcia JM, Gruart A (2004b) Relative contributions of eyelid and eye-retraction motor systems to reflex and classically conditioned blink responses in the rabbit. J Appl Physiol (1985) 96:1541-1554.

Lewis JL, Lo Turco JJ, Solomon PR (1987) Lesions of the middle cerebellar peduncle disrupt acquisition and retention of the rabbit's classically conditioned nictitating membrane response. Behav Neurosci 101:151-157.

Li Z, Murthy VN (2001) Visualizing postendocytic traffic of synaptic vesicles at hippocampal synapses. Neuron 31:593-605.

Lincoln JS, McCormick DA, Thompson RF (1982) Ipsilateral cerebellar lesions prevent learning of the classically conditioned nictitating membrane/eyelid response. Brain Res 242:190-193.

Marr D (1969) A theory of cerebellar cortex. J Physiol 202:437-470.

Marra V, Burden JJ, Thorpe JR, Smith IT, Smith SL, Hausser M, Branco T, Staras K (2012) A preferentially segregated recycling vesicle pool of limited size supports neurotransmission in native central synapses. Neuron 76:579-589.

Martina M, Gorfinkel Y, Halman S, Lowe JA, Periyalwar P, Schmidt CJ, Bergeron R (2004) Glycine transporter type 1 blockade changes NMDA receptor-mediated responses and LTP in hippocampal CA1 pyramidal cells by altering extracellular glycine levels. J Physiol 557:489-500.

Masugi-Tokita M, Tarusawa E, Watanabe M, Molnar E, Fujimoto K, Shigemoto R (2007) Number and density of AMPA receptors in individual synapses in the rat cerebellum as revealed by SDS-digested freeze-fracture replica labeling. J Neurosci 27:2135-2144.

Matsushita M, Iwahori N (1971a) Structural organization of the fastigial nucleus. II. Afferent fiber systems. Brain Res 25:611-624.

Matsushita M, Iwahori N (1971b) Structural organization of the interpositus and the dentate nuclei. Brain Res 35:17-36.

Matsushita M, Ikeda M (1976) Projections from the lateral reticular nucleus to the cerebellar cortex and nuclei in the cat. Exp Brain Res 24:403-421. 
Mauk MD, Steinmetz JE, Thompson RF (1986) Classical conditioning using stimulation of the inferior olive as the unconditioned stimulus. Proc Natl Acad Sci U S A 83:5349-5353.

May PJ, Vidal PP, Baker H, Baker R (2012) Physiological and anatomical evidence for an inhibitory trigemino-oculomotor pathway in the cat. J Comp Neurol 520:2218-2240.

Mayhew TM (1979) Stereological approach to the study of synapse morphometry with particular regard to estimating number in a volume and on a surface. J Neurocytol 8:121-138.

McCarthy KM, Tank DW, Enquist LW (2009) Pseudorabies virus infection alters neuronal activity and connectivity in vitro. PLoS Pathog 5:e1000640.

McCormick DA, Thompson RF (1984a) Neuronal responses of the rabbit cerebellum during acquisition and performance of a classically conditioned nictitating membrane-eyelid response. J Neurosci 4:28112822.

McCormick DA, Thompson RF (1984b) Cerebellum: essential involvement in the classically conditioned eyelid response. Science 223:296-299.

McCormick DA, Lavond DG, Thompson RF (1982a) Concomitant classical conditioning of the rabbit nictitating membrane and eyelid responses: correlations and implications. Physiol Behav 28:769-775.

McCormick DA, Lavond DG, Thompson RF (1983) Neuronal responses of the rabbit brainstem during performance of the classically conditioned nictitating membrane (NM)/eyelid response. Brain Res 271:73-88.

McCormick DA, Steinmetz JE, Thompson RF (1985) Lesions of the inferior olivary complex cause extinction of the classically conditioned eyeblink response. Brain Res 359:120-130.

McCormick DA, Clark GA, Lavond DG, Thompson RF (1982b) Initial localization of the memory trace for a basic form of learning. Proc Natl Acad Sci U S A 79:2731-2735.

McDonald AJ, Pearson JC (1989) Coexistence of GABA and peptide immunoreactivity in non-pyramidal neurons of the basolateral amygdala. Neurosci Lett 100:53-58.

McNeal DW, Ge J, Herrick JL, Stilwell-Morecraft KS, Morecraft RJ (2008) Characterization of some morphological parameters of orbicularis oculi motor neurons in the monkey. Neuroscience 151:12-27.

Mettenleiter TC (2002) Herpesvirus assembly and egress. J Virol 76:1537-1547.

Mettenleiter TC, Klupp BG, Granzow H (2006) Herpesvirus assembly: a tale of two membranes. Curr Opin Microbiol 9:423-429.

Miyata S, Itoh T, Matsushima O, Nakashima T, Kiyohara T (1994) Not only osmotic stress but also repeated restraint stress causes structural plasticity in the supraoptic nucleus of the rat hypothalamus. Brain Res Bull 33:669-675.

Modney BK, Hatton GI (1989) Multiple synapse formation: a possible compensatory mechanism for increased cell size in rat supraoptic nucleus. J Neuroendocrinol 1:21-27. 
Mojtahedian S, Kogan DR, Kanzawa SA, Thompson RF, Lavond DG (2007) Dissociaton of conditioned eye and limb responses in the cerebellar interpositus. Physiol Behav 91:9-14.

Molinari HH, Schultze KE, Strominger NL (1996) Gracile, cuneate, and spinal trigeminal projections to inferior olive in rat and monkey. J Comp Neurol 375:467-480.

Molineux ML, Mehaffey WH, Tadayonnejad R, Anderson D, Tennent AF, Turner RW (2008) Ionic factors governing rebound burst phenotype in rat deep cerebellar neurons. J Neurophysiol 100:2684-2701.

Montarolo PG, Palestini M, Strata P (1982) The inhibitory effect of the olivocerebellar input on the cerebellar Purkinje cells in the rat. J Physiol 332:187-202.

Moore JW (2002) A Neuroscientist's Guide to Classical Conditioning: Springer New York.

Morcuende S, Delgado-Garcia JM, Ugolini G (2002) Neuronal premotor networks involved in eyelid responses: retrograde transneuronal tracing with rabies virus from the orbicularis oculi muscle in the rat. $\mathrm{J}$ Neurosci 22:8808-8818.

Morishita W, Sastry BR (1993) Long-term depression of IPSPs in rat deep cerebellar nuclei. Neuroreport 4:719-722.

Mostofi A, Holtzman T, Grout AS, Yeo CH, Edgley SA (2010) Electrophysiological localization of eyeblink-related microzones in rabbit cerebellar cortex. J Neurosci 30:8920-8934.

Mozzachiodi R, Byrne JH (2010) More than synaptic plasticity: role of nonsynaptic plasticity in learning and memory. Trends Neurosci 33:17-26.

Murphy EH, Brown J, Iannuzzelli PG, Baker R (1990) Regeneration and soma size changes following axotomy of the trochlear nerve. J Comp Neurol 295:685-697.

Nakano H, Masuda K, Sasaki S, Katsuta S (1997) Oxidative enzyme activity and soma size in motoneurons innervating the rat slow-twitch and fast-twitch muscles after chronic activity. Brain Res Bull 43:149-154.

Norman RJ, Buchwald JS, Villablanca JR (1977) Classical conditioning with auditory discrimination of the eye blink in decerebrate cats. Science 196:551-553.

Norman RJ, Villablanca JR, Brown KA, Schwafel JA, Buchwald JS (1974) Classical eyeblink conditioning in the bilaterally hemispherectomized cat. Exp Neurol 44:363-380.

Nusser Z, Cull-Candy S, Farrant M (1997) Differences in synaptic GABA(A) receptor number underlie variation in GABA mini amplitude. Neuron 19:697-709.

O'Leary JL, Smith JM, Inukai J, Mejia HH (1972) Architectonics of the cerebellar nuclei in the rabbit. J Comp Neurol 144:399-428.

Oakley DA, Russell IS (1972) Neocortical lesions and Pavlovian conditioning. Physiol Behav 8:915-926.

Onodera S (1984) Olivary projections from the mesodiencephalic structures in the cat studied by means of axonal transport of horseradish peroxidase and tritiated amino acids. J Comp Neurol 227:37-49. 
Ottersen OP, Storm-Mathisen J (1984) Glutamate- and GABA-containing neurons in the mouse and rat brain, as demonstrated with a new immunocytochemical technique. J Comp Neurol 229:374-392.

Ottersen OP, Storm-Mathisen J, Somogyi P (1988) Colocalization of glycine-like and GABA-like immunoreactivities in Golgi cell terminals in the rat cerebellum: a postembedding light and electron microscopic study. Brain Res 450:342-353.

Ouardouz M, Sastry BR (2000) Mechanisms underlying LTP of inhibitory synaptic transmission in the deep cerebellar nuclei. J Neurophysiol 84:1414-1421.

Pacheco-Calderon R, Carretero-Guillen A, Delgado-Garcia JM, Gruart A (2012) Red nucleus neurons actively contribute to the acquisition of classically conditioned eyelid responses in rabbits. J Neurosci 32:12129-12143.

Palkovits M, Mezey E, Hamori J, Szentagothai J (1977) Quantitative histological analysis of the cerebellar nuclei in the cat. I. Numerical data on cells and on synapses. Exp Brain Res 28:189-209.

Pavlov IP (1927) Les réflexes conditionnels. Étude objective de l'activité nerveuse supérieure des animaux. Paris: F. Alcan.

Pellegrini JJ, Horn AK, Evinger C (1995) The trigeminally evoked blink reflex. I. Neuronal circuits. Exp Brain Res 107:166-180.

Perrett SP, Mauk MD (1995) Extinction of conditioned eyelid responses requires the anterior lobe of cerebellar cortex. J Neurosci 15:2074-2080.

Person AL, Raman IM (2010) Deactivation of L-type Ca current by inhibition controls LTP at excitatory synapses in the cerebellar nuclei. Neuron 66:550-559.

Pickard GE, Smeraski CA, Tomlinson CC, Banfield BW, Kaufman J, Wilcox CL, Enquist LW, Sollars PJ (2002) Intravitreal injection of the attenuated pseudorabies virus PRV Bartha results in infection of the hamster suprachiasmatic nucleus only by retrograde transsynaptic transport via autonomic circuits. J Neurosci 22:2701-2710.

Pijpers A, Voogd J, Ruigrok TJ (2005) Topography of olivo-cortico-nuclear modules in the intermediate cerebellum of the rat. J Comp Neurol 492:193-213.

Pomeranz LE, Reynolds AE, Hengartner CJ (2005) Molecular biology of pseudorabies virus: impact on neurovirology and veterinary medicine. Microbiol Mol Biol Rev 69:462-500.

Porras-Garcia E, Sanchez-Campusano R, Martinez-Vargas D, Dominguez-del-Toro E, Cendelin J, Vozeh F, Delgado-Garcia JM (2010) Behavioral characteristics, associative learning capabilities, and dynamic association mapping in an animal model of cerebellar degeneration. J Neurophysiol 104:346-365.

Pugh JR, Raman IM (2005) GABAA receptor kinetics in the cerebellar nuclei: evidence for detection of transmitter from distant release sites. Biophys J 88:1740-1754.

Pugh JR, Raman IM (2006) Potentiation of mossy fiber EPSCs in the cerebellar nuclei by NMDA receptor activation followed by postinhibitory rebound current. Neuron 51:113-123. 
Pugh JR, Raman IM (2008) Mechanisms of potentiation of mossy fiber EPSCs in the cerebellar nuclei by coincident synaptic excitation and inhibition. J Neurosci 28:10549-10560.

Pugh JR, Raman IM (2009) Nothing can be coincidence: synaptic inhibition and plasticity in the cerebellar nuclei. Trends Neurosci 32:170-177.

Radpour S (1977) Organization of the facial nerve nucleus in the cat. Laryngoscope 87:557-574.

Rall W (1967) Distinguishing theoretical synaptic potentials computed for different soma-dendritic distributions of synaptic input. J Neurophysiol 30:1138-1168.

Raman IM, Gustafson AE, Padgett D (2000) Ionic currents and spontaneous firing in neurons isolated from the cerebellar nuclei. J Neurosci 20:9004-9016.

Ramnani N, Yeo CH (1996) Reversible inactivations of the cerebellum prevent the extinction of conditioned nictitating membrane responses in rabbits. J Physiol 495 ( Pt 1):159-168.

Ramón y Cajal S (1888) Sobre las fibras nerviosas de la capa molecular del cerebelo. Rev Trim Histol Normal Patol 1:33-49.

Ramón y Cajal S (1889) Sobre las fibras nerviosas de la capa granulosa del cerebelo. Rev Trim Histol Normal Patol 1:107-118.

Ramón y Cajal S (1894) The Croonian Lecture: La fine structure des centres nerveux. Proceedings of the Royal Society of London 55:444-468.

Rasmussen A, Jirenhed DA, Hesslow G (2008) Simple and complex spike firing patterns in Purkinje cells during classical conditioning. Cerebellum (London, England) 7:563-566.

Robleto K, Thompson RF (2008) Extinction of a classically conditioned response: red nucleus and interpositus. J Neurosci 28:2651-2658.

Roche M, Commons KG, Peoples A, Valentino RJ (2003) Circuitry underlying regulation of the serotonergic system by swim stress. J Neurosci 23:970-977.

Roettger VR, Pearson JC, Goldfinger MD (1989) Identification of gamma-aminobutyric acid-like immunoreactive neurons in the rat cuneate nucleus. Neurosci Lett 97:46-50.

Rosen DJ, Steinmetz JE, Thompson RF (1989) Classical discrimination conditioning of the rabbit's eyelid response using pontine stimulation as a conditioned stimulus. Behav Neural Biol 52:51-62.

Rosenfield ME, Moore JW (1985) Red nucleus lesions impair acquisition of the classically conditioned nictitating membrane response but not eye-to-eye savings or unconditioned response amplitude. Behav Brain Res 17:77-81.

Rubelowski JM, Menge M, Distler C, Rothermel M, Hoffmann KP (2013) Connections of the superior colliculus to shoulder muscles of the rat: a dual tracing study. Front Neuroanat 7:17.

Rubio ME, Juiz JM (2004) Differential distribution of synaptic endings containing glutamate, glycine, and GABA in the rat dorsal cochlear nucleus. J Comp Neurol 477:253-272. 
Ruigrok TJ (2011) Ins and outs of cerebellar modules. Cerebellum (London, England) 10:464-474.

Ruigrok TJ, Voogd J (2000) Organization of projections from the inferior olive to the cerebellar nuclei in the rat. J Comp Neurol 426:209-228.

Rush AN, Robinette BL, Stanton ME (2001) Ontogenetic differences in the effects of unpaired stimulus preexposure on eyeblink conditioning in the rat. Dev Psychobiol 39:8-18.

Russ JC, Dehoff RT (2000) Practical Stereology. Boston, MA: Springer US.

Salm AK, Modney BK, Hatton GI (1988) Alterations in supraoptic nucleus ultrastructure of maternally behaving virgin rats. Brain Res Bull 21:685-691.

Sanchez-Campusano R, Gruart A, Fernandez-Mas R, Delgado-Garcia JM (2012) An agonist-antagonist cerebellar nuclear system controlling eyelid kinematics during motor learning. Front Neuroanat 6:8-8.

Sanchez M, Sillitoe RV, Attwell PJ, Ivarsson M, Rahman S, Yeo CH, Hawkes R (2002)

Compartmentation of the rabbit cerebellar cortex. J Comp Neurol 444:159-173.

Sastry BR, Morishita W, Yip S, Shew T (1997) GABA-ergic transmission in deep cerebellar nuclei. Prog Neurobiol 53:259-271.

Schikorski T, Stevens CF (1997) Quantitative ultrastructural analysis of hippocampal excitatory synapses. J Neurosci 17:5858-5867.

Schikorski T, Stevens CF (2001) Morphological correlates of functionally defined synaptic vesicle populations. Nat Neurosci 4:391-395.

Schild RF (1970) On the inferior olive of the albino rat. J Comp Neurol 140:255-260.

Schmaltz LW, Theios J (1972) Acquisition and extinction of a classically conditioned response in hippocampectomized rabbits (Oryctolagus cuniculus). J Comp Physiol Psychol 79:328-333.

Schmitz SK, Hjorth JJ, Joemai RM, Wijntjes R, Eijgenraam S, de Bruijn P, Georgiou C, de Jong AP, van Ooyen A, Verhage M, Cornelisse LN, Toonen RF, Veldkamp WJ (2011) Automated analysis of neuronal morphology, synapse number and synaptic recruitment. J Neurosci Methods 195:185-193.

Schneiderman N, Fuentes I, Gormezano I (1962) Acquisition and extinction of the classically conditioned eyelid response in the albino rabbit. Science 136:650-652.

Schonewille M, Gao Z, Boele HJ, Veloz MF, Amerika WE, Simek AA, De Jeu MT, Steinberg JP, Takamiya K, Hoebeek FE, Linden DJ, Huganir RL, De Zeeuw CI (2011) Reevaluating the role of LTD in cerebellar motor learning. Neuron 70:43-50.

Schreurs BG, Alkon DL (2001) Imaging learning and memory: classical conditioning. Anat Rec 265:257273.

Schreurs BG, Sanchez-Andres JV, Alkon DL (1991) Learning-specific differences in Purkinje-cell dendrites of lobule HVI (Lobulus simplex): intracellular recording in a rabbit cerebellar slice. Brain Res 548:18-22. 
Schreurs BG, Tomsic D, Gusev PA, Alkon DL (1997) Dendritic excitability microzones and occluded long-term depression after classical conditioning of the rabbit's nictitating membrane response. J Neurophysiol 77:86-92.

Schreurs BG, Shi T, Pineda S, 3rd, Buck DL (2000) Conditioning the unconditioned response: modification of the rabbit's (Oryctolagus cuniculus) unconditioned nictitating membrane response. J Exp Psychol Anim Behav Process 26:144-156.

Schreurs BG, Gusev PA, Tomsic D, Alkon DL, Shi T (1998) Intracellular correlates of acquisition and long-term memory of classical conditioning in Purkinje cell dendrites in slices of rabbit cerebellar lobule HVI. J Neurosci 18:5498-5507.

Seal RP, Edwards RH (2006) Functional implications of neurotransmitter co-release: glutamate and GABA share the load. Curr Opin Pharmacol 6:114-119.

Sears LL, Steinmetz JE (1991) Dorsal accessory inferior olive activity diminishes during acquisition of the rabbit classically conditioned eyelid response. Brain Res 545:114-122.

Shaikh AG, Ghasia FF, Dickman JD, Angelaki DE (2005) Properties of cerebellar fastigial neurons during translation, rotation, and eye movements. J Neurophysiol 93:853-863.

Shinoda Y, Sugihara I, Wu HS, Sugiuchi Y (2000) The entire trajectory of single climbing and mossy fibers in the cerebellar nuclei and cortex. Prog Brain Res 124:173-186.

Siebold C, Glonti L, Glasauer S, Buttner U (1997) Rostral fastigial nucleus activity in the alert monkey during three-dimensional passive head movements. J Neurophysiol 77:1432-1446.

Sillitoe RV, Marzban H, Larouche M, Zahedi S, Affanni J, Hawkes R (2005) Conservation of the architecture of the anterior lobe vermis of the cerebellum across mammalian species. Prog Brain Res 148:283-297.

Simat M, Parpan F, Fritschy JM (2007) Heterogeneity of glycinergic and gabaergic interneurons in the granule cell layer of mouse cerebellum. J Comp Neurol 500:71-83.

Smith BN, Banfield BW, Smeraski CA, Wilcox CL, Dudek FE, Enquist LW, Pickard GE (2000)

Pseudorabies virus expressing enhanced green fluorescent protein: A tool for in vitro electrophysiological analysis of transsynaptically labeled neurons in identified central nervous system circuits. Proc Natl Acad Sci U S A 97:9264-9269.

Smith MC, Coleman SR, Gormezano I (1969) Classical conditioning of the rabbit's nictitating membrane response at backward, simultaneous, and forward CS-US intervals. J Comp Physiol Psychol 69:226-231.

Soghomonian JJ, Sethares C, Peters A (2010) Effects of age on axon terminals forming axosomatic and axodendritic inhibitory synapses in prefrontal cortex. Neuroscience 168:74-81.

Song CK, Enquist LW, Bartness TJ (2005) New developments in tracing neural circuits with herpesviruses. Virus Res 111:235-249.

Song G, Wang H, Xu H, Poon CS (2012) Kolliker-Fuse neurons send collateral projections to multiple hypoxia-activated and nonactivated structures in rat brainstem and spinal cord. Brain Struct Funct. 
Sotelo C, Angaut P (1973) The fine structure of the cerebellar central nuclei in the cat. I. Neurons and neuroglial cells. Exp Brain Res 16:410-430.

Stanic D, Kuteeva E, Nylander I, Hokfelt T (2010) Characterization of CGRP protein expression in "satellite-like" cells and dendritic arbours of the mouse olfactory bulb. J Comp Neurol 518:770-784.

Steinmetz JE, Lavond DG, Thompson RF (1989) Classical conditioning in rabbits using pontine nucleus stimulation as a conditioned stimulus and inferior olive stimulation as an unconditioned stimulus. Synapse 3:225-233.

Steinmetz JE, Logue SF, Steinmetz SS (1992) Rabbit classically conditioned eyelid responses do not reappear after interpositus nucleus lesion and extensive post-lesion training. Behav Brain Res 51:103-114.

Steinmetz JE, Rosen DJ, Woodruff-Pak DS, Lavond DG, Thompson RF (1986a) Rapid transfer of training occurs when direct mossy fiber stimulation is used as a conditioned stimulus for classical eyelid conditioning. Neurosci Res 3:606-616.

Steinmetz JE, Rosen DJ, Chapman PF, Lavond DG, Thompson RF (1986b) Classical conditioning of the rabbit eyelid response with a mossy-fiber stimulation CS: I. Pontine nuclei and middle cerebellar peduncle stimulation. Behav Neurosci 100:878-887.

Steinmetz JE, Logan CG, Rosen DJ, Thompson JK, Lavond DG, Thompson RF (1987) Initial localization of the acoustic conditioned stimulus projection system to the cerebellum essential for classical eyelid conditioning. Proc Natl Acad Sci U S A 84:3531-3535.

Stigger F, do Nascimento PS, Dutra MF, Couto GK, Ilha J, Achaval M, Marcuzzo S (2011) Treadmill training induces plasticity in spinal motoneurons and sciatic nerve after sensorimotor restriction during early postnatal period: new insights into the clinical approach for children with cerebral palsy. Int J Dev Neurosci 29:833-838.

Sugihara I (2011) Compartmentalization of the deep cerebellar nuclei based on afferent projections and aldolase C expression. Cerebellum (London, England) 10:449-463.

Sugihara I, Shinoda Y (2007) Molecular, topographic, and functional organization of the cerebellar nuclei: analysis by three-dimensional mapping of the olivonuclear projection and aldolase $\mathrm{C}$ labeling. $\mathrm{J}$ Neurosci 27:9696-9710.

Sugihara I, Quy PN (2007) Identification of aldolase C compartments in the mouse cerebellar cortex by olivocerebellar labeling. J Comp Neurol 500:1076-1092.

Sugihara I, Wu HS, Shinoda Y (2001) The entire trajectories of single olivocerebellar axons in the cerebellar cortex and their contribution to Cerebellar compartmentalization. J Neurosci 21:7715-7723.

Sugihara I, Fujita H, Na J, Quy PN, Li BY, Ikeda D (2009) Projection of reconstructed single Purkinje cell axons in relation to the cortical and nuclear aldolase $\mathrm{C}$ compartments of the rat cerebellum. J Comp Neurol 512:282-304.

Sultan F, Konig T, Mock M, Thier P (2002) Quantitative organization of neurotransmitters in the deep cerebellar nuclei of the Lurcher mutant. J Comp Neurol 452:311-323. 
Sun LW (2012) Transsynaptic tracing of conditioned eyeblink circuits in the mouse cerebellum. Neuroscience 203:122-134.

Svensson P, Jirenhed DA, Bengtsson F, Hesslow G (2010) Effect of conditioned stimulus parameters on timing of conditioned Purkinje cell responses. J Neurophysiol 103:1329-1336.

Swenson RS, Castro AJ (1983) The afferent connections of the inferior olivary complex in rats: a study using the retrograde transport of horseradish peroxidase. Am J Anat 166:329-341.

Tadayonnejad R, Anderson D, Molineux ML, Mehaffey WH, Jayasuriya K, Turner RW (2010) Rebound discharge in deep cerebellar nuclear neurons in vitro. Cerebellum (London, England) 9:352-374.

Takada M, Itoh K, Yasui Y, Mitani A, Nomura S, Mizuno N (1984) Distribution of premotor neurons for orbicularis oculi motoneurons in the cat, with particular reference to possible pathways for blink reflex. Neurosci Lett 50:251-255.

Tarnecki R, Zurawska I (1989) Functional relations between neighboring neurons of nucleus interpositus of the cat cerebellum. Acta Physiol Pol 40:456-472.

Tarusawa E, Matsui K, Budisantoso T, Molnar E, Watanabe M, Matsui M, Fukazawa Y, Shigemoto R (2009) Input-specific intrasynaptic arrangements of ionotropic glutamate receptors and their impact on postsynaptic responses. J Neurosci 29:12896-12908.

Telgkamp P, Raman IM (2002) Depression of inhibitory synaptic transmission between Purkinje cells and neurons of the cerebellar nuclei. J Neurosci 22:8447-8457.

Telgkamp P, Padgett DE, Ledoux Va, Woolley CS, Raman IM (2004) Maintenance of high-frequency transmission at purkinje to cerebellar nuclear synapses by spillover from boutons with multiple release sites. Neuron 41:113-126.

Teune TM, van der Burg J, Ruigrok TJ (1995) Cerebellar projections to the red nucleus and inferior olive originate from separate populations of neurons in the rat: a non-fluorescent double labeling study. Brain Res 673:313-319.

Teune TM, van der Burg J, van der Moer J, Voogd J, Ruigrok TJ (2000) Topography of cerebellar nuclear projections to the brain stem in the rat. Prog Brain Res 124:141-172.

Thompson RF (1988) The neural basis of basic associative learning of discrete behavioral responses. Trends Neurosci 11:152-155.

Thompson RF (2013) An essential memory trace found. Behav Neurosci 127:669-675.

Tolbert DL, Massopust LC, Murphy MG, Young PA (1976) The anatomical organization of the cerebello-olivary projection in the cat. J Comp Neurol 170:525-544.

Tomishima MJ, Smith GA, Enquist LW (2001) Sorting and transport of alpha herpesviruses in axons. Traffic 2:429-436. 
Tracy JA, Britton GB, Steinmetz JE (2001) Comparison of single unit responses to tone, light, and compound conditioned stimuli during rabbit classical eyeblink conditioning. Neurobiol Learn Mem 76:253-267.

Travers JB, Rinaman L (2002) Identification of lingual motor control circuits using two strains of pseudorabies virus. Neuroscience 115:1139-1151.

Uchizono K (1965) Characteristics of excitatory and inhibitory synapses in the central nervous system of the cat. Nature 207:642-643.

Ugolini G (2011) Rabies virus as a transneuronal tracer of neuronal connections. Adv Virus Res 79:165202.

Uusisaari M, Knopfel T (2010) GlyT2+ neurons in the lateral cerebellar nucleus. Cerebellum (London, England) 9:42-55.

Uusisaari M, Knopfel T (2011) Functional classification of neurons in the mouse lateral cerebellar nuclei. Cerebellum (London, England) 10:637-646.

Uusisaari M, Obata K, Knopfel T (2007) Morphological and electrophysiological properties of GABAergic and non-GABAergic cells in the deep cerebellar nuclei. J Neurophysiol 97:901-911.

Van Daele DJ, Cassell MD (2009) Multiple forebrain systems converge on motor neurons innervating the thyroarytenoid muscle. Neuroscience 162:501-524.

Van Daele DJ, Fazan VP, Agassandian K, Cassell MD (2011) Amygdala connections with jaw, tongue and laryngo-pharyngeal premotor neurons. Neuroscience 177:93-113.

van den Pol AN (1991) Glutamate and aspartate immunoreactivity in hypothalamic presynaptic axons. J Neurosci 11:2087-2101.

Van der Want JJ, Wiklund L, Guegan M, Ruigrok T, Voogd J (1989) Anterograde tracing of the rat olivocerebellar system with Phaseolus vulgaris leucoagglutinin (PHA-L). Demonstration of climbing fiber collateral innervation of the cerebellar nuclei. J Comp Neurol 288:1-18.

van Ham JJ, Yeo CH (1996a) The central distribution of primary afferents from the external eyelids, conjunctiva, and cornea in the rabbit, studied using WGA-HRP and B-HRP as transganglionic tracers. Exp Neurol 142:217-225.

van Ham JJ, Yeo CH (1996b) Trigeminal inputs to eyeblink motoneurons in the rabbit. Exp Neurol 142:244-257.

VanderWerf F, Aramideh M, Otto JA, Ongerboer de Visser BW (1998) Retrograde tracing studies of subdivisions of the orbicularis oculi muscle in the rhesus monkey. Exp Brain Res 121:433-441.

Villar-Cervino V, Abalo XM, Villar-Cheda B, Melendez-Ferro M, Perez-Costas E, Holstein GR, Martinelli GP, Rodicio MC, Anadon R (2006) Presence of glutamate, glycine, and gamma-aminobutyric acid in the retina of the larval sea lamprey: comparative immunohistochemical study of classical neurotransmitters in larval and postmetamorphic retinas. J Comp Neurol 499:810-827. 
Vogel RW, Amundson JC, Lindquist DH, Steinmetz JE (2009) Eyeblink conditioning during an interstimulus interval switch in rabbits (Oryctolagus cuniculus) using picrotoxin to disrupt cerebellar cortical input to the interpositus nucleus. Behav Neurosci 123:62-74.

Voneida TJ, Christie D, Bogdanski R, Chopko B (1990) Changes in instrumentally and classically conditioned limb-flexion responses following inferior olivary lesions and olivocerebellar tractotomy in the cat. J Neurosci 10:3583-3593.

Voogd J, Ruigrok TJ (1997) Transverse and longitudinal patterns in the mammalian cerebellum. Prog Brain Res 114:21-37.

Voogd J, Glickstein M (1998) The anatomy of the cerebellum. Trends Neurosci 21:370-375.

Wang D, Schreurs BG (2006) Characteristics of IA currents in adult rabbit cerebellar Purkinje cells. Brain Res 1096:85-96.

Wang D, Schreurs BG (2010) Dietary cholesterol modulates the excitability of rabbit hippocampal CA1 pyramidal neurons. Neurosci Lett 479:327-331.

Wang SS, Khiroug L, Augustine GJ (2000) Quantification of spread of cerebellar long-term depression with chemical two-photon uncaging of glutamate. Proc Natl Acad Sci U S A 97:8635-8640.

Wassef M, Simons J, Tappaz ML, Sotelo C (1986) Non-Purkinje cell GABAergic innervation of the deep cerebellar nuclei: a quantitative immunocytochemical study in C57BL and in Purkinje cell degeneration mutant mice. Brain Res 399:125-135.

Weeks ACW, Connor S, Hinchcliff R, LeBoutillier JC, Thompson RF, Petit TL (2007) Eye-blink conditioning is associated with changes in synaptic ultrastructure in the rabbit interpositus nuclei. Learn Mem 14:385-389.

West MJ (1999) Stereological methods for estimating the total number of neurons and synapses: issues of precision and bias. Trends Neurosci 22:51-61.

West MJ (2012) Introduction to stereology. Cold Spring Harb Protoc 2012:843-851.

Whealy ME, Baumeister K, Robbins AK, Enquist LW (1988) A herpesvirus vector for expression of glycosylated membrane antigens: fusion proteins of pseudorabies virus gIII and human immunodeficiency virus type 1 envelope glycoproteins. J Virol 62:4185-4194.

Williams SR, Stuart GJ (2003) Role of dendritic synapse location in the control of action potential output. Trends Neurosci 26:147-154.

Woodruff-Pak DSS, Joseph E. (2000) Eyeblink Classical Conditioning, Volume 1 : Applications in Humans. Hingham, MA, USA: Kluwer Academic Publishers.

Wu XY, Zhu JX, Gao J, Owyang C, Li Y (2005) Neurochemical phenotype of vagal afferent neurons activated to express C-FOS in response to luminal stimulation in the rat. Neuroscience 130:757-767. 
Yang BY, Weisz DJ (1992) An auditory conditioned stimulus modulates unconditioned stimulus-elicited neuronal activity in the cerebellar anterior interpositus and dentate nuclei during nictitating membrane response conditioning in rabbits. Behav Neurosci 106:889-899.

Yeo CH, Hesslow G (1998) Cerebellum and conditioned reflexes. Trends Cogn Sci 2:322-330.

Yeo CH, Hardiman MJ, Glickstein M (1985a) Classical conditioning of the nictitating membrane response of the rabbit. III. Connections of cerebellar lobule HVI. Exp Brain Res 60:114-126.

Yeo CH, Hardiman MJ, Glickstein M (1985b) Classical conditioning of the nictitating membrane response of the rabbit. II. Lesions of the cerebellar cortex. Exp Brain Res 60:99-113.

Yokota T, Koyama N, Nishikawa Y, Nishikawa N, Nishida Y, Hasegawa A, Fujino Y (1991) Trigeminal nociceptive neurons in the subnucleus reticularis ventralis. I. Response properties and afferent connections. Neurosci Res 11:1-17.

Zhang W, Shin JH, Linden DJ (2004) Persistent changes in the intrinsic excitability of rat deep cerebellar nuclear neurones induced by EPSP or IPSP bursts. J Physiol 561:703-719.

Zheng N, Raman IM (2010) Synaptic inhibition, excitation, and plasticity in neurons of the cerebellar nuclei. Cerebellum (London, England) 9:56-66. 